\title{
ASTEROID LIGHT CURVES FROM THE PALOMAR TRANSIENT FACTORY SURVEY: ROTATION PERIODS AND PHASE FUNCTIONS FROM SPARSE PHOTOMETRY
}

\author{
Adam WaszczaK ${ }^{1}$, Chan-Kao Chang $^{2}$, Eran O. Ofek ${ }^{3}$, Russ Laher ${ }^{4}$, Frank Masci ${ }^{5}$, David Levitan ${ }^{6}$, Jason Surace ${ }^{4}$, \\ Yu-Chi Cheng ${ }^{2}$, Wing-Huen IP ${ }^{2}$, Daisuke Kinoshita ${ }^{2}$, George Helou ${ }^{5}$, Thomas A. Prince ${ }^{6}$, and Shrinivas Kulkarni ${ }^{6}$ \\ ${ }^{1}$ Division of Geological and Planetary Sciences, California Institute of Technology, Pasadena, CA 91125, USA; waszczak@caltech.edu \\ ${ }^{2}$ Institute of Astronomy, National Central University, Jhongli, Taiwan \\ ${ }^{3}$ Benoziyo Center for Astrophysics, Weizmann Institute of Science, Rehovot, Israel \\ ${ }^{4}$ Spitzer Science Center, California Institute of Technology, Pasadena, CA 91125, USA \\ ${ }^{5}$ Infrared Processing and Analysis Center, California Institute of Technology, Pasadena, CA 91125, USA \\ ${ }^{6}$ Division of Physics, Mathematics and Astronomy, California Institute of Technology, Pasadena, CA 91125, USA \\ Received 2015 March 4; accepted 2015 April 15; published 2015 August 18
}

\begin{abstract}
We fit 54,296 sparsely sampled asteroid light curves in the Palomar Transient Factory survey to a combined rotation plus phase-function model. Each light curve consists of 20 or more observations acquired in a single opposition. Using 805 asteroids in our sample that have reference periods in the literature, we find that the reliability of our fitted periods is a complicated function of the period, amplitude, apparent magnitude, and other light-curve attributes. Using the 805-asteroid ground-truth sample, we train an automated classifier to estimate (along with manual inspection) the validity of the remaining 53,000 fitted periods. By this method we find that 9033 of our light curves (of $~ 8300$ unique asteroids) have "reliable" periods. Subsequent consideration of asteroids with multiple light-curve fits indicates a 4\% contamination in these "reliable" periods. For 3902 light curves with sufficient phase-angle coverage and either a reliable fit period or low amplitude, we examine the distribution of several phase-function parameters, none of which are bimodal though all correlate with the bond albedo and with visible-band colors. Comparing the theoretical maximal spin rate of a fluid body with our amplitude versus spin-rate distribution suggests that, if held together only by self-gravity, most asteroids are in general less dense than $\sim 2 \mathrm{~g} \mathrm{~cm}^{-3}$, while $\mathrm{C}$ types have a lower limit of between 1 and $2 \mathrm{~g} \mathrm{~cm}^{-3}$. These results are in agreement with previous density estimates. For 5-20 km diameters, $S$ types rotate faster and have lower amplitudes than $\mathrm{C}$ types. If both populations share the same angular momentum, this may indicate the two types' differing ability to deform under rotational stress. Lastly, we compare our absolute magnitudes (and apparent-magnitude residuals) to those of the Minor Planet Center's nominal ( $G=0.15$, rotation-neglecting) model; our phase-function plus Fourier-series fitting reduces asteroid photometric rms scatter by a factor of $\sim 3$.
\end{abstract}

Key words: minor planets, asteroids: general - surveys

Supporting material: FITS file, machine-readable and VO tables

\section{INTRODUCTION}

In this work we model an asteroid's apparent visual magnitude $V(\log$ flux $)$ as

$$
V=H+\delta+5 \log _{10}(r \Delta)-2.5 \log _{10}[\phi(\alpha)],
$$

where $H$ is the absolute magnitude (a constant), $\delta$ is a periodic variability term due to rotation (e.g., if the object is spinning and has some asymmetry in shape or albedo), $r$ and $\Delta$ are the heliocentric and geocentric distances (in AU), and $\phi=\phi(\alpha)$ is the phase function, which varies with the solar phase angle $\alpha$ (the Sun-asteroid-Earth angle). When $\alpha=0$ (i.e., at opposition), $\phi=1$ by definition, while in general $0<\phi<1$ for $\alpha>0$ (with $\phi$ decreasing as $\alpha$ increases).

A key feature of our approach is the simultaneous fitting of both the phase function $\phi$ and the rotation term $\delta$. The detailed forms of $\phi$ and $\delta$, as well as the algorithm underlying our fitting procedure, are motivated by a variety of prior work in this area, as described in the following sections.

\subsection{Asteroid Rotation}

Building upon the work of Kaasalainen et al. (2001), Hanuš \& Durech (2012) discuss the inversion of asteroid light-curve data taken over several oppositions to obtain a 3D shape solution. The form of $\delta$ (see Equation (1)) in this case consists of a large number of free parameters (several tens to hundreds). Results from inversion agree well with those from stellar occultations, adaptive optics imaging, and in situ spacecraft imagery (Hanuš et al. 2013). Knowledge of the detailed irregular shapes of asteroids improves our ability to constrain models of their internal structure, as well as the magnitude and timescale of spin and orbital evolution due to solar-radiation and thermal emission, including the Yarkovsky and YORP effects (see Bottke et al. 2013 and references therein).

A simpler model for $\delta$-suitable for fitting to data sparser than those required for most inversion methods-is a Jacobi ellipsoid (Chandrasekhar 1969) in its principal-axis spin state. The light curve of such an ellipsoid is a double-peaked sinusoid, given by a simple expression depending solely (assuming constant surface albedo) on the axes ratio and angle between the line of sight and spin axis. The fitted amplitude thus yields a lower-bound elongation estimate for the asteroid.

The predicted distribution of the rotation frequencies of a collisionally equilibrated system of particles has long been claimed to be a Maxwellian function (Salo 1987), which-as reviewed by Pravec et al. (2002) - very well approximates the observed distribution of several hundred of the brightest 
( $40 \mathrm{~km}$ or larger) asteroids, but breaks down for smaller objects, among which an excess of slow and fast rotators appear to exist. Steinberg \& Sari (2015) more recently argue that collision instead leads to a Lévy distribution, and that a significant primordial spin component remains in the present observed population. Some studies that have examined the spin distribution of small objects are Pravec et al. (2008), Polishook \& Borsch (2009), the Thousand Asteroid Light Curve Survey (Masiero et al. 2009), and two brief observing runs conducted within the Palomar Transient Factory ${ }^{7}$ (PTF) survey (Polishook et al. 2012; Chang et al. 2014a).

Warner et al. (2009) describe the Light Curve Database (LCDB), which compiles several thousand densely sampled light curves of asteroids targeted by dedicated observing teams. Light curves in the LCDB have the following features:

1. LCDB light curves' dense sampling generally permits fitting of Fourier series with many harmonic terms;

2. LCDB light curves are often sampled over the shortest time window necessary to measure the period and therefore generally do not require large or uncertain corrections due to phase-angle effects;

3. LCDB light curves' fitted periods are assigned integer quality codes by a human reviewer (from $1=$ poor to $3=$ confident $)$.

All three of the above features are either impractical or infeasible when the set of light curves is very large and the data sparsely sampled, as is the case for PTF. In this work we adopt the following modified approaches when fitting light curves:

1. We truncate the rotation curve's Fourier-series fit after the 2nd harmonic, a simplification broadly justified by Harris et al. (2014) and the assumption of an ellipsoidal shape (see Section 3.1.2).

2. We simultaneously fit a phase-function model with the rotational part.

3. We use a machine-learned classifier to objectively aid in estimating the validity of each fitted period. The classifier is trained using all fitted light curves that have previously (and confidently) measured LCDB periods and takes into account the accuracy with which the true period was retrieved along with 20 light-curve metrics (fitted period, amplitude, ratio of peaks, $\chi^{2}$ per degree of freedom of fit, number of data points, and more).

Use of a machine classifier in asteroid light-curve period quality assessment is entirely novel and inspired in part by work done by PTF collaborators in extragalactic transient science (Bloom et al. 2012) and variable star science (Masci et al. 2014; Miller et al. 2014), as well as work by A. Waszczak et al. (2015, in preparation) on detection techniques for streaking near-Earth objects. Among the advantages of using a machine-classified quality score is that, via cross-validation with the known-period sample, one estimates the completeness and contamination, i.e., the true-positive rate (TPR) and falsepositive rate (FPR) with respect to identifying an accurately fit period, as a function of, e.g., the period, amplitude, etc. The resulting TPR and FPR may then be used to de-bias the classifier-filtered period distribution.

\footnotetext{
http://ptf.caltech.edu
}

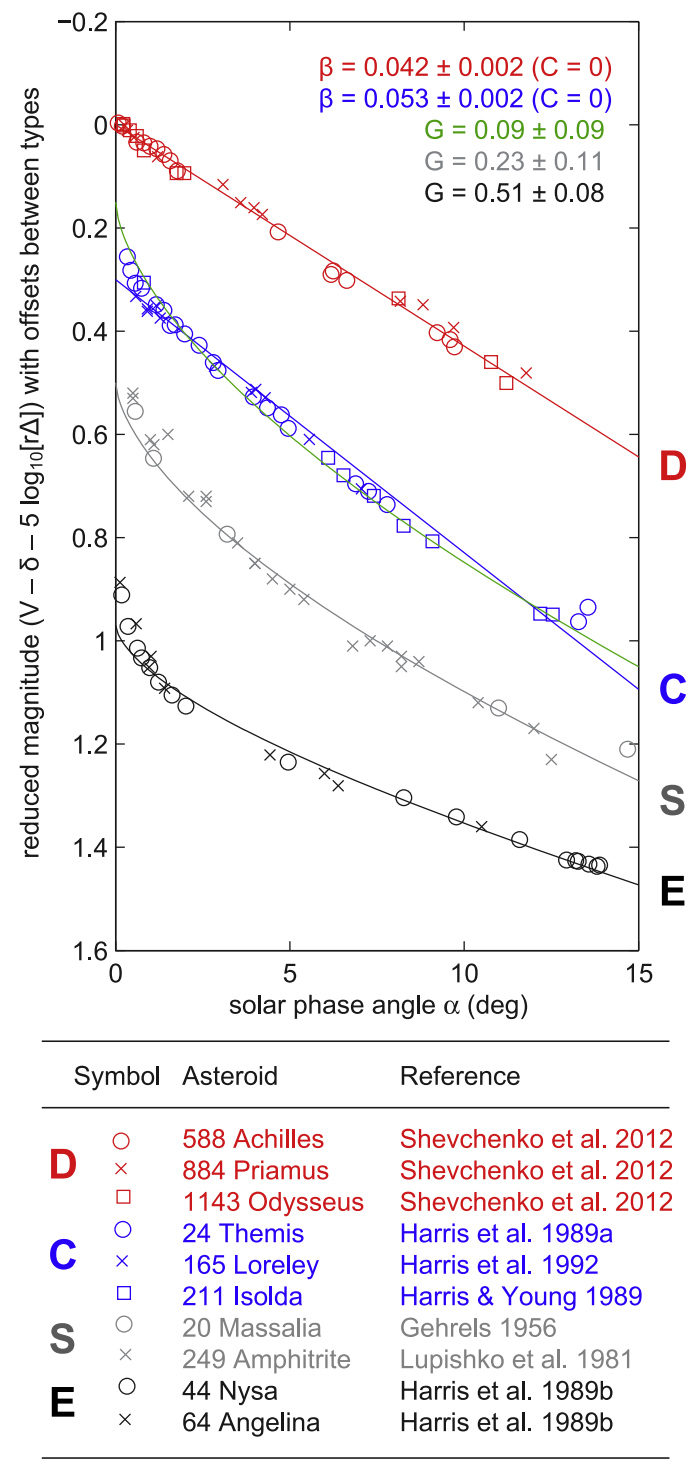

Figure 1. Phase curves (from the literature) containing densely sampled, rotation-corrected photometry of asteroids in four taxonomic classes. Colored lines are our original fits to the data using various single-parameter $\phi$ models (see Section 3.2).

\subsection{Asteroid Phase Functions}

The analytic phase function of an ideal Lambertianscattering sphere fits well to featureless, atmospheric planets like Venus, but quite poorly to airless bodies (see Figure 3.9 of Seager 2010 for a comparison). In later sections we describe several $\phi$ models that have been derived for (or empirically fit to) asteroids. Qualitatively, asteroids show an approximately linearly decreasing $\phi$ out to $\alpha \approx 100^{\circ}$, modified by a surge (increase in slope) at low phase angles $\left(\alpha \lesssim 5^{\circ}\right)$, known as the opposition effect (see Figure 1).

Early work (e.g., Bowell et al. 1989 and references therein) on a small sample of well-observed asteroids suggested that different asteroid spectral types display distinct behavior in $\phi$. Figure 1 compares example phase-curve data for D, C, S, and E types, ${ }^{8}$ incorporating photometry from various sources. We emphasize the fact that all of the data points in Figure 1 have

\footnotetext{
8 Bus et al. (2002) review these and other asteroid taxonomic classes, which are defined on the basis of low-resolution $(R \approx 100)$ visible reflectance spectra.
} 
been corrected for rotational modulation (the $\delta$ in Equation (1)) through dense sampling of each asteroid's light curve at each phase angle (equivalently, each epoch).

Using a large corpus of low-precision photometry from the MPC, ${ }^{9}$ Oszkiewicz et al. $(2011,2012)$ showed that a fitted parameter of one particular $\phi$ model correlates well with an asteroid's Sloan Digital Sky Survey (SDSS) visible color. While they were unable to correct for rotational variation $(\delta$ term in Equation (1)), the Oszkiewicz et al. work nevertheless demonstrates a solid trend between $\phi$ and a compositional attribute (color).

These prior works motivate several defining aspects of this work's phase-function analysis:

1. We fit multiple phase-function models to each light curve, both for compatibility with the literature and to explore how the fitted parameters are related.

2. We simultaneously fit the rotational component with the phase-function part.

3. We introduce a single colorimetric index for quantifying C-type versus S-type taxonomic classification, based on the compilation of several visible-band-color asteroid data sets (see Appendix), and examine the variation in phasefunction parameters as a function of this color index.

\section{OBSERVATIONS}

\subsection{Overview of the PTF Survey}

The PTF is a synoptic survey designed primarily to discover extragalactic transients (Law et al. 2009; Rau et al. 2009). The PTF camera, mounted on Palomar Observatory's $1.2 \mathrm{~m}$ Oschin Schmidt Telescope, uses $11 \mathrm{CCDs}$ (each $2 \mathrm{~K} \times 4 \mathrm{~K}$ ) to image $7.3 \mathrm{deg}^{2}$ of sky at a time at $1 .{ }^{\prime \prime} 0 /$ pixel resolution. Most exposures $(\sim 85 \%)$ use a Mould- $R$ filter ${ }^{10}$ (hereafter “ $R$ "). The remaining broadband images acquired use a Gunn $g$-band filter. Nearly all broadband PTF images are $60 \mathrm{~s}$ integrations, regardless of filter. About $15 \%$ of nights (near full moon) are devoted to a narrowband $(\mathrm{H} \alpha)$ imaging survey of the full Northern Sky.

Science operations began in 2009 March, with a nominal one- to five-day cadence for supernova discovery and typical twice-per-night imaging of fields. Median seeing is $2^{\prime \prime}$ with a limiting magnitude $R \approx 20.5$ (for $5 \sigma$ point-source detections), while dark conditions routinely yield $R \approx 21.0$ (Law et al. 2010).

The PTF survey is ongoing and expected to continue through mid-2016. In January 2013 the PTF project formally entered a second phase called the intermediate PTF ("iPTF'; Kulkarni 2013). In this paper we simply use "PTF" to mean the entire survey, from 2009 through the present (2015). The iPTF program accommodates more varied "sub-surveys" as opposed to a predominantly extragalactic program, including variable star and solar system science. Images are still acquired with the same telescope/camera/filters with $60 \mathrm{~s}$ exposures and are processed by the same reduction pipeline.

Laher et al. (2014) describe the PTF data reduction and archiving pipelines, hosted at the Infrared Processing and Analysis Center (IPAC) at Caltech. Processing at IPAC includes bias and flat-field corrections, astrometric calibration

\footnotetext{
9 IAU Minor Planet Center, http://minorplanetcenter.net.

10 The Mould- $R$ filter is very similar to the SDSS- $r$ filter; see Ofek et al. (2012a) for its transmission curve.
}

against UCAC3 (Zacharias 2010), astrometric verification against Two Micron All Sky Survey (2MASS; Skrutskie et al. 2006), creation of source catalogs with Source Extractor (Bertin \& Arnouts 1996), and production of reference images (stacks of $\sim 20-30$ PTF images that reach $V \approx 22$ ).

Ofek et al. (2012a, 2012b) describe the PTF survey's absolute photometric calibration method, which relies on source matching with SDSS DR7 (Abazajian et al. 2009), and thus requires at least partial overlap of PTF with SDSS each night. A separate, relative photometric calibration (based on light curves of non-variable field stars) also exists for PTF data and is described by Levitan et al. (2011) and in the Appendix of Ofek et al. (2011). In this work we utilize all $R$ band and $g$-band PTF data accumulated from the survey's start (2009 March) through 2014 July. The asteroid magnitudes reported in this work use relative photometric zero points when available (which as of this writing applies to $\sim 85 \%$ of PTF images) and absolute photometric zero points otherwise.

The PTF's robotic survey program and processing pipeline, as well as our data aggregation and analysis in this work, make use of many functions from the MATLAB package for astronomy and astrophysics (Ofek 2014).

\subsection{This Work's Data Set}

Waszczak et al. (2013) used a custom spatial indexing algorithm to search the set of all PTF single-epoch transient detections (through 2012 July) for detections of all asteroids with orbits known as of 2012 August. That search procedure first generated uniformly spaced ephemerides for each asteroid using JPL's online service (HORIZONS; Giorgini et al. 1996). Each asteroid's ephemeris defines a 3D curve (two sky coordinates plus one time); the intersection of each curve with the 3D kd-tree of transient detections was then computed and positive detections within a $4^{\prime \prime}$ matching radius saved.

In this work we use a modified version of the Waszczak et al. (2013) algorithm. The updates/changes are as follows.

First, in terms of content, we now search all PTF $(R$ and $g$ band) data from 2009 March 01 through 2014 July 18 for all numbered asteroids as of 2014 July 12 (401,810 objects). We now exclude unnumbered objects as the positional uncertainty of these objects can be very large, and as they tend to be very faint, their light curves will not in general be of high quality.

Second, in place of a single-step matching of a 3D transientdetection kd-tree against 3D ephemeris curves, we now divide the search into two main steps. We first perform a 2D spatial matching that exploits the natural indexing of PTF exposures into tiles (i.e., the grid of evenly spaced boresights or "fields" on the sky). Each 2D ephemeris curve's intersection with the 2D PTF survey footprint is computed, the object's position is cubically interpolated to all epochs of exposures possibly containing the object, and the object's precisely computed positon is then compared to the precise image boundaries of candidate exposures. Matching of predicted positions against actual detections takes place subsequently as source catalogs are then loaded into memory (as needed and in parallel). This method is faster than the original Waszczak et al. (2013) method and enables separate logging of predicted and positive detections.

The results of the known-asteroid search, as well as the derived light-curve data (described later), are stored in a relational database, the size and contents of which are summarized in Table 1 . Out of $\sim 18$ million predicted single- 
Table 1

Description of the PTF Asteroid Database

\begin{tabular}{|c|c|c|}
\hline Table & \# Rows & Example Columns (not necessarily comprehensive) \\
\hline PTF tiles & 11,169 & R.A., Decl., tile ID \\
\hline Exposures & 304,982 & Epoch, filter, exposure time, absolute photometric zero point, tile ID, exposure ID \\
\hline CCD images & $3,305,426$ & CCD ID, corners R.A. and Decl., seeing, limiting mag., relative phot. zero point, \# of sources, exposure ID, image ID \\
\hline Asteroids & 401,810 & Name, orbital elements, color data (e.g., SDSS), IR data (e.g., WISE), known rotation period, asteroid ID (number) \\
\hline Predicted sightings & $17,929,274$ & R.A., Decl., rates, helio- and geocentric range, phase and elong. angle, pred. $V$ mag., image ID, asteroid ID, prediction ID \\
\hline Positive detections & $8,842,305$ & R.A., Decl., instrumental mag., local zero point, shape data, quality flags, prediction ID, light-curve ID, detection ID \\
\hline Reliable detections ${ }^{\mathrm{a}}$ & $4,392,395$ & Detection ID \\
\hline Light curves $^{\mathrm{b}}$ & 587,466 & \# of constituent detections, filter, opposition year, median mag., asteroid ID, light-curve ID \\
\hline Light-curve fits ${ }^{c}$ & 54,296 & Fitted light-curve parameters, human-assigned quality code, machine-classified quality index, light-curve ID, fit ID \\
\hline Reliable-period fits ${ }^{\mathrm{d}}$ & 9033 & Fit ID \\
\hline Reliable- $G_{12}$ fits $^{\mathrm{e}}$ & 3902 & Fit ID \\
\hline
\end{tabular}

Note. Includes PTF data acquired from 2009 March through 2014 July, excluding H $\alpha$ survey data.

a "Reliable" detections are those free from possible background-source or bright star contamination, magnitude errors $>0.1$ mag, and certain SExtractor flags.

${ }^{\mathrm{b}}$ A light curve is here defined as a set of positive detections of a given asteroid in a single filter and opposition.

${ }^{\mathrm{c}}$ Light-curve fits only exist for light curves that contain at least 20 reliable detections and converged to a solution during the light-curve-fitting process.

${ }^{\mathrm{d}}$ Fits have reliable rotation periods if a human screener labels the period reliable and the machine classifier rates it above a certain quality threshold (see text).

${ }^{\mathrm{e}}$ Fits have reliable $G_{12}$ phase-function parameter if (1) amplitude $<0.1 \mathrm{mag}$ or period is reliable, and (2) fit has sufficient phase-angle coverage (see Section 6.3).
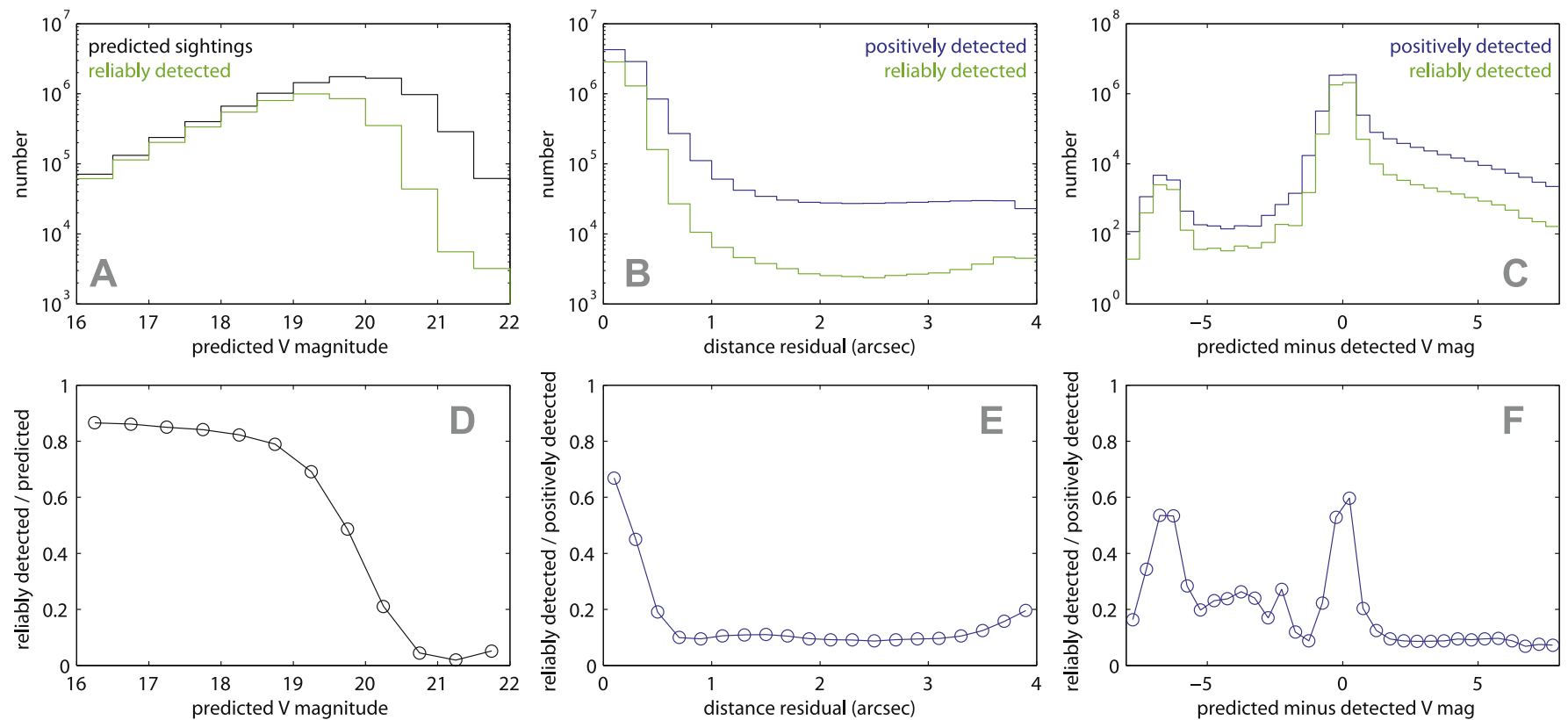

Figure 2. Comparison of predicted asteroid sightings against positive and "reliable" asteroid detections. We define a "reliable" detection as any positive detection that (1) lacks any cataloged background sources within a 4 " radius, (2) has a calibrated magnitude uncertainty of less than 0.1 mag, and (3) lacks any processing warning flags. As suggested by the middle and right column of plots, this definition of "reliable" still contains some small contamination (at the $<1 \%$ level) from uncataloged background sources and/or noise, as indicated by detections with distance residuals greater than $\sim 1^{\prime \prime}$ or magnitude residuals of greater than $\sim 1$ mag. In panel $\mathrm{D}$, the less than $100 \%$ completeness at the bright end reflects the non-negligible probability that any asteroid will fall within $4^{\prime \prime}$ of a cataloged background source (regardless of the magnitude of either the asteroid or the background source).

epoch asteroid sightings (including predicted magnitudes as $\operatorname{dim}$ as $V \approx 23$, well below PTF's sensitivity), there were 8.8 million positive detections (within a $4^{\prime \prime}$ radius). Of these, we define 4.3 million detections as "reliable" as they (1) lack any cataloged background sources within the 4 " radius, (2) have a calibrated magnitude uncertainty of less than $0.1 \mathrm{mag}$, and (3) lack any processing flags indicative of contamination. Figure 2 compares predicted, positive, and "reliable" detections; the middle and right panels of Figure 2 show that our definition of "reliable" seems to include a small fraction of likely bad observations $(<1 \%$ contamination, note the vertical log scale), namely, those that have distance residuals greater than $\sim 1^{\prime \prime}$ or magnitude residuals greater than $\sim 1$ mag. Because these reliable detections are the subset of observations that we input into our light-curve-fitting model (Section 4), the fitting algorithm includes logic designed to remove isolated data points that have very large residuals, either with respect to the median light curve value or relative to their uncertainty.

\section{LIGHT-CURVE MODEL}

Equation (1) presents the overall form and notation of our asteroid light-curve model. In this section we describe the detailed parameterization and assumptions of the model. 


\subsection{Rotation Component}

\subsubsection{Intra-opposition Constraint}

The most important parameter in the rotation component (the $\delta$ in Equation (1)) is the synodic spin period $P$, a constant that satisfies

$$
\delta(\tau)=\delta(\tau+n P),
$$

where $\tau \equiv t-\Delta / c$ is the light-time-corrected observation timestamp, $\Delta=\Delta(t)$ is the asteroid's geocentric distance, $c$ is the speed of light, and $n$ is any integer satisfying

$$
|n| \ll P_{\text {orb }} / P
$$

where $P_{\text {orb }}$ is the synodic orbital period,

$$
P_{\text {orb }}=\left(\frac{1}{\mathrm{yr}}-\frac{1}{T_{\mathrm{orb}}}\right)^{-1}=\left(\frac{1}{\mathrm{yr}}-\frac{\sqrt{G M_{\odot}}}{2 \pi a_{\mathrm{orb}}^{3 / 2}}\right)^{-1},
$$

where $T_{\text {orb }}$ is the asteroid's sidereal orbital period and $a_{\text {orb }}$ is its orbital semimajor axis (related by Kepler's third law). $P_{\text {orb }}$ is the time elapsed between the asteroid's consecutive oppositions. Pursuant to this restriction, we constrain each $\delta$ solution using observations from within the same opposition-i.e., for most asteroids, within a 1.1-1.6 yr interval centered on the date of locally minimally observed $\alpha$.

The intra-opposition restriction is important given that our data set (described in the next section) spans $\sim 5 \mathrm{yr}$. For an asteroid with a zero-inclination circular orbit and spin axis perpendicular to its orbital plane, we can relax Equation (3) to allow $n$ to be any integer, in which case $\delta$ can be constrained using observations spanning many years. In general, however, Equation (2) must be modified to accommodate a varying viewing geometry with respect to the spin axis:

$$
\delta(\tau)=F(\tau) \delta(\tau+n P),
$$

where $F$ is some unknown periodic function satisfying $F(t)=F\left(t+m T_{\text {orb }}\right)$, where $m$ is any integer and $T_{\text {orb }}$ is the sidereal orbital period. Provided that the amplitude of $F$ is not large relative to that of $\delta$, and provided that the spin vector is not changing with respect to the orbital plane (i.e., precessing ${ }^{11}$ ) on a timescale comparable to $P_{\mathrm{orb}}$, we are justified in assuming that Equation (2) (with the Equation (3) restriction) applies.

\subsubsection{Second-order Fourier Series}

Any $\delta$ satisfying Equation (2) can be approximated to arbitrary precision using a Fourier series. Harris et al. (2014) discuss why, from a geometric standpoint, the second harmonic tends to dominate an asteroid's fitted $\delta$. As noted earlier (Section 1.1), most large asteroids approximately resemble triaxial prolate ellipsoids (e.g., Jacobi ellipsoids), having equatorial axis ratios of at most $\sim 3: 1$ (corresponding to a $\delta_{\max }-\delta_{\min }$ amplitude of $\sim 1.2 \mathrm{mag}$ ). For less extreme axis ratios (specifically, those producing a $\sim 0.4 \mathrm{mag}$ or smaller second-harmonic amplitude), other harmonics related to shape or albedo asymmetries may contribute comparable coefficients to the Fourier approximation of $\delta$.

\footnotetext{
11 Principal-axis rotation (a stable equilibrium state) is assumed for most planetary bodies. Burns \& Safronov (1973) discuss the relevant timescales of spin evolution.
}

The PTF survey program has-on a few rare occasionsconducted high-cadence ( $\sim 10$ minutes spaced) observations of low ecliptic latitude fields. These runs produced a set of $\sim 1000$ densely sampled main-belt asteroid rotation curves, which have already been analyzed and published (Polishook et al. 2012; Chang et al. 2014a). These high-cadence "pilot studies" are relevant to our present work in that they demonstrate (1) the quality of the PTF survey's photometric calibration for asteroids with unambiguously valid $\delta$ solutions and (2) the above-described prevalence of a dominant second harmonic in most of the objects sampled.

Following these pilot studies, we adopt a second-order Fourier series model:

$$
\delta \equiv \sum_{k=1,2} A_{1, k} \sin \left(\frac{2 \pi k \tau}{P}\right)+A_{2, k} \cos \left(\frac{2 \pi k \tau}{P}\right)
$$

where $\tau$ is the light-time-corrected epoch (see Equation (2)). In the pilot studies, most of the fitted $\delta$ solutions qualitatively resemble a simple sine or cosine function. Such a solution can be represented by either

1. a first harmonic with period $P=P_{1}$ (with $A_{i, 1} \neq 0$ and $\left.A_{i, 2}=0\right)$, or

2. a second harmonic of period $P=\frac{1}{2} P_{1}$ (with $A_{i, 1}=0$ and $\left.A_{i, 2} \neq 0\right)$.

Given the prolate ellipsoid model, choice 2 is more realistic and hence preferred. However, again recognizing that other harmonics can have a non-negligible contribution, in fitting $\delta$ to our light-curve sample we allow the first-harmonic coefficients $A_{i, 1}$ to be non-zero, but introduce logic into the fitting algorithm (see Section 4), which checks for doubleperiod solutions satisfying certain criteria and iterates accordingly.

\subsection{Phase-function Component}

In this work we simultaneously fit each light curve's phase function $\phi$ along with its rotation curve $\delta$ (see Equation (1)). This approach is intermediate in complexity between some of the simpler, two-parameter ( $\delta$-neglecting) models that have been applied to very large data sets (e.g., Oszkiewicz et al. 2012; Williams 2012) and the more complex, shape plus poleorientation models (Kaasalainen 2004; Cellino et al. 2009; Hanuš \& Durech 2012) which can involve tens of parameters and require data spanning multiple oppositions.

Regarding the former class of models, we note that there is a formal statistical problem associated with neglecting $\delta$ when fitting $\phi$. If modeling the observations $M$ by $V^{\prime} \equiv V-\delta=H+5 \log _{10}(r \Delta)-2.5 \log _{10}(\phi)$, then the distribution of residuals $M-V^{\prime}$ is not Gaussian. Assuming that $\delta$ is a sinusoid with amplitude $A$, for observations $M$ sampling the light curve at random times, the residual probability density function $p=p\left(M-V^{\prime}\right)$ has a local minimum value $p_{\min }$ at $M-V^{\prime}=0$ and maximum value $p_{\max }$ near $M-V^{\prime}= \pm \mathrm{A}$. Thus, $p$ is bimodal and roughly bowl shaped-not at all Gaussian shaped. The uncertainty in $\phi$ produced by a standard $\chi^{2}$ minimization - which assumes Gaussian-distributed errors -is thus inaccurate. However, since $p$ is symmetric about $M-V^{\prime}=0$, for densely sampled data the fitted phase function $\phi$ remains unaffected by neglecting $\delta$; in such a case the only effect is an underestimated uncertainty. 
We obtain three separate fits for each light curve, each using a different phase function $(\phi)$ and allowing for unique solutions for $H$ and $\delta$ in Equation (1). The three phasefunction models are:

1. the two-parameter model of Shevchenko (1997);

2. the one-parameter $G$ model (Bowell et al. 1989);

3. the one-parameter $G_{12}$ model (Muinonen et al. 2010).

In this section we review and motivate the application of each of these $\phi$ models.

\subsubsection{Two-parameter Shevchenko Model}

Shevchenko (1997) introduced a phase function dependent on two parameters; in terms of Equation (1) the model is ${ }^{12}$

$$
-2.5 \log _{10}[\phi(\alpha)] \equiv \beta \alpha-C \frac{\alpha}{1+\alpha},
$$

where $\beta$ has units of mag/deg and $C$ is the amplitude of the opposition surge (units of mag). This model was subsequently considered in-depth by Belskaya \& Shevchenko (2000, hereafter $\mathrm{B} \& \mathrm{~S}$ ), who compiled the most complete (to date) set of high-precision, targeted phase-curve observations of main-belt asteroids from various data sets spanning several decades.

Though in practice Shevchenko's model is the least commonly used phase function out of the three we consider, it is by far the simplest to express mathematically, and it is the only model for $\phi$ whose parameters have linear dependence in Equation (1).

Furthermore, this model's parameters are the most straightforward to associate with physical asteroid properties. B\&S highlighted a robust relationship between an asteroid's $(\beta, C)$ phase-function parameters and its geometric albedo. ${ }^{13}$ As we later explore a similar relationship in the present work, we here review the basis of this observation.

The geometric albedo $p_{V}$ is formally defined in terms of the phase function $\phi$ :

$$
p_{V} \equiv \frac{A_{\text {bond }}}{2}\left(\int_{0}^{\pi} \phi(\alpha) \sin (\alpha) d \alpha\right)^{-1} \equiv \frac{A_{\text {bond }}}{q},
$$

where $A_{\text {bond }}$ is the (visible) bond albedo, defined as the total visible light energy reflected or scattered by the asteroid (in all directions) divided by the total visible light energy incident upon the asteroid (from the Sun). We also here define the phase integral $q$.

$\mathrm{B} \& \mathrm{~S}$ showed that, in the range of $\beta$ observed from S-type to C-type asteroids, $\beta$ and $C$ are empirically correlated, in a relation that we approximate here as

$$
C \approx(0.9 \mathrm{mag})-\left(17^{\circ}\right) \beta \text { for } 0.03<\frac{\beta}{\mathrm{mag} / \mathrm{deg}}<0.05 .
$$

Using Equation (9) to substitute for $C$ in Equation (7), inserting the result into Equation (8), and numerically

\footnotetext{
12 In Shevchenko's original notation, $\beta$ is denoted $b$ and $C$ is denoted $a$. Moreover, in the original notation, $\phi(0)=-a$; we here added a constant term $+a$ to make $\phi(0)=1$, following convention with other phase functions.

13 Also known as the visible albedo or the physical albedo.
}

evaluating the integral gives

$$
\begin{gathered}
p_{V} \approx A_{\text {bond }}\left(0.4-\frac{2.2 \beta}{\mathrm{mag} / \mathrm{deg}}\right) \\
\text { for } 0.03<\frac{\beta}{\mathrm{mag} / \mathrm{deg}}<0.05 .
\end{gathered}
$$

$\mathrm{B} \& \mathrm{~S}$ saw a negative correlation between $p_{V}$ and $\beta$ in the data, ${ }^{14}$ consistent with Equation (10) only if either $A_{\text {bond }}$ is assumed constant among different asteroid types (not a reasonable assumption) or if $A_{\text {bond }}$ negatively correlates with $\beta$, which $\mathrm{B} \& \mathrm{~S}$ did not explicitly show.

The bond albedo $A_{\text {bond }}$ can be thought of as an intrinsic, bulk-compositional characteristic of an asteroid's surface, ${ }^{15}$ much like an asteroid's color, whereas $\beta$ and $C$ relate (in part) to the textural, particulate, and macroscopic roughness of the asteroid's surface. B\&S and other authors separately associate $\beta$ with the shadow-hiding effect and $C$ with the coherent backscatter effect. Both of these physical phenomena are understood from a theoretical standpoint (e.g., Helfenstein \& Veverka 1989; Hapke 2012) to be functions of $A_{\text {bond }}$, with $\beta$ negatively related to $A_{\text {bond }}$ and $C$ positively related. This is consistent with Equation (9) and renders Equation (10) consistent with B\&S's noted $p_{V}$-versus- $\beta$ correlation. Other properties such as particle size, particle geometry, and regolith porosity also have predicted (and laboratory-measured) contributions to the observed phase function (Hapke 2012 and references therein); these properties can conceivably vary independently of $A_{\text {bond }}$.

In short, our interpretation of the S-type and C-type asteroid data reviewed by $\mathrm{B} \& \mathrm{~S}$ is that a compositional indicator $\left(A_{\text {bond }}\right)$ correlates with indicators of two independent phenomena $(\beta$ and $C$ ) that contribute to how light scatters from an asteroid's surface. This statement intentionally makes no mention of $p_{V}$, since Equation (8) tells us that $p_{V}$ by definition varies with $\beta$ (in a non-obvious way) and with $A_{\text {bond }}$, the latter being a more basic compositional attribute.

As stated above, the phase function can be related to properties other than $A_{\text {bond }}$, such as regolith porosity. Many of these other properties in theory and experiment contribute to effects involving multiply scattered light and therefore do not alter the effect of shadow hiding ( $\beta$-term in Equation (7)), which is dominated by singly scattered light (Hapke 2012). In contrast, the coherent backscatter effect $(\boldsymbol{C}$-term) does involve multiply scattered light. B\&S saw non-monotonic behavior in $C$ as a function of $p_{V}$ when including the rarer, high- $p_{V}$ E-type asteroids in the same plot as $\mathrm{C}$ and $\mathrm{S}$ types. E types do conform, however, to the same negative monotonic trend in $p_{V^{-}}$ versus- $\beta$ satisfied by the $\mathrm{C}$ and $\mathrm{S}$ types, consistent with the hypothesis that $\beta$ is adequately expressed as a function of $A_{\text {bond }}$ alone, yet $\mathrm{E}$ types have a lower-than-predicted $C$ value based on extrapolation of Equation (9).

One possibility is that Equation (9) is not valid for all asteroids, but must be replaced by some unknown nonmonotonic relationship, possibly because $C$ depends nonmonotonically on $A_{\text {bond }}$ and/or has comparable dependence on

\footnotetext{
${ }^{14} \mathrm{~B} \& \mathrm{~S}$ actually stated the correlation in terms of $\log p_{V}$ versus $\beta$, though the range in $\beta$ is sufficiently small that $p_{V}$ versus $\beta$ is essentially valid as well.

15 More accurately, the single-scattering albedo $w$, which is the analog of $A_{\text {bond }}$ for a "point-source" particle, more fundamentally embodies this bulkcompositional attribute. Hapke (2012) details how $A_{\text {bond }}$ is solely a function of $w$ for an asteroid whose surface consists of isotropic scatterers; we here use $A_{\text {bond }}$ as a proxy for $w$.
} 
other properties (e.g., porosity or grain size). Assuming that Equation (7) is a sufficiently general model for $\phi$, and lacking knowledge of a good model for $C$, it follows that $\beta$ and $C$ should in practice always be fit separately. Another possibility is that Equation (7) is an incorrect or incomplete model; however, B\&S described no instances wherein their model was unable to adequately fit the data for a particular asteroid or class of asteroids.

\subsubsection{Lumme-Bowell G Model}

The next phase-function model we consider is the LummeBowell model (Bowell et al. 1989), also known as the $(H, G)$ or IAU phase function:

$$
\left\{\begin{aligned}
\phi & \equiv(1-G) \phi_{1}+G \phi_{2} \\
\phi_{1} & \equiv \exp \left(-3.33 \tan ^{0.63}[\alpha / 2]\right) \\
\phi_{2} & \equiv \exp \left(-1.87 \tan ^{1.22}[\alpha / 2]\right) .
\end{aligned}\right.
$$

Like Shevchenko's model, this model includes two terms (the basis functions $\phi_{1}$ and $\phi_{2}$ ) representing two physically distinct contributions to the observed $\phi$. As detailed in Bowell et al. (1989), this model is semi-empirical in that it was derived from basic principles of radiative transfer theory with certain assumptions, and at various stages tailored to match existing laboratory and astronomical observations. That the two basis functions' coefficients are related to a single parameter $G$ bears resemblance to the $\beta$-versus- $C$ correlation described by Equation (9).

Marsden (1986) marked the IAU's adoption of this phase function as a standard model for predicting an asteroid's brightness. Since then this model has seen widespread application, and it is often used with the assumption $G=0.15$ (e.g., in the ephemeris computation services offered by the MPC and JPL). Harris \& Young (1988) present mean values of $G$ for several of the major asteroid taxonomic classes (based on a sample of $\sim 80$ asteroids), with $G=0.15$ being an average between the $\mathrm{C}$ types $(G \approx 0.08)$ and the $\mathrm{S}$ types $(G \approx 0.23)$. The $G$-model fails to accurately fit the rarer $\mathrm{D}$ types (which have linear phase curves) and E types (which have very sharp opposition spikes), whereas the Shevchenko model can properly accommodate these rarer types.

Use of the Lumme-Bowell $\phi$ in our light-curve model (Equation (1)) introduces a second nonlinear parameter $(G)$ into the model, the period $P$ being the other nonlinear parameter. This complicates the fitting algorithm somewhat, as described in Section 4.

\subsubsection{Muinonen et al. $G_{12}$ Model}

The third phase-function model we consider, introduced by Muinonen et al. (2010), bears resemblance to the $G$-model but includes a second free parameter and a third basis function:

$$
\phi \equiv G_{1} \phi_{1}+G_{2} \phi_{2}+\left(1-G_{1}-G_{2}\right) \phi_{3} .
$$

As opposed to the analytic trigonometric basis functions of the $G$-model, here $\phi_{1}, \phi_{2}$, and $\phi_{3}$ (all functions of $\alpha$ alone) are defined in terms of cubic splines (see Muinonen et al. 2010 for the exact numerical definitions). Assuming that the coefficients $G_{1}$ and $G_{2}$ are constrained independently, these basis functions were designed to provide the most accurate fits to the phase functions of all major asteroid taxonomic types, including the rarer D types and E types.

For situations where fitting $G_{1}$ and $G_{2}$ separately is infeasible, Muinonen et al. (2010) specialized their above model to make it a function of a single parameter, $G_{12}$, which parameterizes $G_{1}$ and $G_{2}$ using piecewise functions:

$$
\begin{gathered}
G_{1}= \begin{cases}0.7527 G_{12}+0.06164 & \text { if } G_{12}<0.2 ; \\
0.9529 G_{12}+0.02162 & \text { otherwise; }\end{cases} \\
G_{2}= \begin{cases}-0.9612 G_{12}+0.6270 & \text { if } G_{12}<0.2 ; \\
-0.6125 G_{12}+0.5572 & \text { otherwise }\end{cases}
\end{gathered}
$$

In this work we use this single-parameter $G_{12}$ form of the Muinonen et al. model, making it analogous to the $G$-model in terms of implementation, including the complication associated with a nonlinear parameter.

\subsubsection{Multi-parameter Hapke Model}

Just as we commented on the more rigorous means of fitting a rotation curve via $3 \mathrm{D}$ shape modeling with multiopposition data, for completeness we note that a more rigorous model (than the three presented above) exists for phase functions. Given better-sampled light curves and more computational power, future modeling of large photometric data sets would benefit from applying the more theoretically motivated model of Hapke (2012), an abbreviated form of which is

$$
\phi=\frac{B_{C} K}{p_{V}}\left[\left(\frac{w}{8}\left(B_{S} g-1\right)+\frac{r_{0}-r_{0}^{2}}{2}\right) h+\frac{2}{3} r_{0}^{2} \phi_{L}\right] .
$$

Here $w$ is the single-scattering albedo (see footnote 15), of which $r_{0}$ is solely a function. The remaining factors all are functions of phase angle $(\alpha)$. Each opposition-surge term $\left(B_{S}\right.$ and $\left.B_{C}\right)$ has two free parameters (width and amplitude). $K$ depends on the mean topographic roughness (a function of one free parameter); $g$ is the single-scattering angular distribution function (typically includes one parameter); $h$ is a function of $\alpha$ only; and $\phi_{L}$ is the phase function of an ideal Lambertian-scattering sphere (a simple function of $\alpha$ ).

With its $\phi \propto p_{V}^{-1}$ dependence, the Hapke model (Equation (14)) can conveniently eliminate both $p_{V}$ and $H$ from the modeling process. Inserting Equation (14) into Equation (1), and using the common relation ${ }^{16}$

$$
H=-5 \log _{10}\left(\frac{D \sqrt{p_{V}}}{1329 \mathrm{~km}}\right),
$$

where $H$ is the absolute visual magnitude, $D$ is the asteroid's effective diameter, and $1329 \mathrm{~km}$ is a constant (set by the arbitrarily defined magnitude of the Sun), produces a model

\footnotetext{
${ }^{16}$ Rather that attributing it to any specific author(s), we note that Equation (15) may be derived directly using Equation (8) and the following definition of the bond albedo, which we stated in words immediately after Equation (8):

$$
A_{\text {bond }} \equiv \frac{\int_{0}^{\pi} 10^{-V(\alpha) / 2.5} \sin (\alpha) d \alpha}{\left(10^{-M \operatorname{Sun} / 2.5} / 4 \pi \mathrm{AU}^{2}\right) \times \pi(D / 2)^{2}},
$$

where $V(\alpha)=H-2.5 \log _{10} \phi(\alpha)$ is Equation (1) evaluated at $\delta=0$ and $r=\Delta=1 \mathrm{AU}$.
} 
with many physically meaningful parameters and free of both $H$ and $p_{V}$.

\section{LIGHT-CURVE-FITTING ALGORITHM}

We solve Equation (1) using a custom linear least squares (LLSq) method. A basic review of LLSq can be found in Hogg et al. (2010). Each fitted asteroid light curve contains $N_{\text {obs }} \geqslant 20$ observations, with measured apparent magnitudes $m_{i}$ and measurement uncertainties $\sigma_{i}$. All instrumental magnitudes are elliptical aperture (Kron 1980) measurements (SExtractor's MAG_AUTO) calibrated with a local zero point (i.e., the "ZPVM" correction of Ofek et al. 2012a). The uncertainties contain a Poisson-noise component (SExtractor's MAGERR_AUTO), as well as systematic error from the calibration. For images lacking a relative photometric solution, the relevant systematic error is the APBSRMS parameter in the PTF database; for images having a relative photometric solution, the systematic error is a combination of the sysErr and zeropointerr database quantities (added in quadrature).

In all cases, our model (Equation (1)) is nonlinear in at least one parameter (the period $P$, or equivalently the frequency $f=1 / P)$. We test $N_{\text {frq }}$ evenly spaced frequencies between $f=0$ (infinite rotation period) and $f=12$ days $^{-1}$, i.e., up to the $\sim 2 \mathrm{hr}$ spin barrier.

Asteroids rotating faster than the $\sim 2 \mathrm{hr}$ spin barrier are likely monolithic objects and-particularly if larger than $\sim 150 \mathrm{~m}-$ are interesting in their own right (see the discussion in Pravec et al. 2002). However, given the apparent observed rarity of such super-fast rotators (SFRs) and the large interval in frequency space that must be searched to discover them; we impose $2 \mathrm{hr}=12$ cycles per day as our upper limit on fitted frequency in order to make computational time reasonable without sacrificing sensitivity to the majority of asteroids' spin rates. Chang et al. (2014a) present preliminary results of an independent, ongoing effort to use PTF data (or at least specific subsets thereof) to search for SFRs, with at least one SFR having been discovered and confirmed (Chang et al. 2014b).

We use a frequency spacing $\Delta f=1 /(4 \Delta t)$, where $\Delta t$ is the time interval between the first and last observation in the light curve. Formally $\Delta t$ can be as long as $1.1-1.6 \mathrm{yr}$ for most asteroids (see Section 3.1.1); however, the median value of $\Delta t$ (among light curves that ultimately acquired fits) is $\sim 45$ days, with 16th and 84th percentiles of 13 and 106 days, respectively.

In addition to the nonlinear parameter $f$, the light-curve model in general has $N_{\text {lin }}$ linear parameters. We seek to solve the following tensor equation for $X$ :

$$
m_{i}=\sum_{j, k} L_{i j k} X_{j k}\left\{\begin{array}{l}
i=1,2, \ldots, N_{\mathrm{obs}} \\
j=1,2, \ldots, N_{\mathrm{frq}} \\
k=1,2, \ldots, N_{\mathrm{lin}}
\end{array}\right.
$$

where $m_{i}$ is the $i$ th observation, $L$ is the "design matrix" (a 3D array of size $N_{\text {obs }} \times N_{\text {frq }} \times N_{\text {lin }}$ ), and $X$ is the linear-parameter matrix $\left(N_{\text {frq }} \times N_{\text {lin }}\right)$ containing the linear-parameter solutions as a function of frequency.

\subsection{Linear Phase-function Parameters}

For the particular case wherein we use Shevchenko's model (Equation (7)) for the phase function $\phi$, the design matrix is

$$
L_{i j}=\left(\begin{array}{c}
1 \\
\sin \left(2 \pi f_{j} \tau_{i}\right) \\
\cos \left(2 \pi f_{j} \tau_{i}\right) \\
\sin \left(4 \pi f_{j} \tau_{i}\right) \\
\cos \left(4 \pi f_{j} \tau_{i}\right) \\
\alpha_{i} \\
\alpha_{i} /\left(1+\alpha_{i}\right)
\end{array}\right)
$$

where the $k$-index has been omitted with the convention that $k=1$ is the first row of the above column vector, $k=2$ is the second row, etc. Here $\tau_{i}$ and $\alpha_{i}$ are the time and phase angle of the $i$ th observation, $f_{j}$ is the $j$ th frequency, etc. Likewise, the linear-parameter matrix $X$ in this case is

$$
X_{j}=\left(\begin{array}{c}
H_{j} \\
\left(A_{1,1}\right)_{j} \\
\left(A_{2,1}\right)_{j} \\
\left(A_{1,2}\right)_{j} \\
\left(A_{2,2}\right)_{j} \\
\beta_{j} \\
C_{j}
\end{array}\right),
$$

where $H_{j}$ is the fitted absolute magnitude for the $j$ th frequency, etc.

The general LLSq solution to Equation (16) is

$$
X_{j k}=\sum_{\ell, n, p} S_{j k \ell} L_{n j \ell}\left(B^{-1}\right)_{n p} m_{p},
$$

where $B^{-1}$ is the inverse of the data-covariance matrix $B$ :

$$
B=\left(\begin{array}{cccc}
\sigma_{1}^{2} & 0 & \cdots & 0 \\
0 & \sigma_{2}^{2} & \cdots & 0 \\
\vdots & \vdots & \ddots & \vdots \\
0 & 0 & \cdots & \sigma_{N_{\mathrm{obs}}}^{2}
\end{array}\right),
$$

and $S_{j k \ell}$ is the parameter-covariance matrix, given by

$$
S_{j k \ell}=\left[\left(s_{j}\right)^{-1}\right]_{k \ell},
$$

where in the above definition we invert each of the $N_{\text {frq }}$ matrices $s_{j}$, these being defined by

$$
\left(s_{j}\right)_{k \ell} \equiv \sum_{n, p} L_{n j k}\left(B^{-1}\right)_{n p} L_{p j \ell} .
$$

The elements of the parameter-covariance matrix $S$ are the variances and covariances of the fitted parameters (as a function of frequency). The fit's residuals (as a function of 
frequency) are

$$
R_{i j}=m_{i}-\sum_{k} L_{i j k} X_{j k}
$$

and the fit's chi-squared (as a function of frequency) is

$$
\left(\chi^{2}\right)_{j}=\sum_{\ell, n} R_{\ell j}\left(B^{-1}\right)_{\ell n} R_{n j} .
$$

The frequency-dependent chi-squared $\left(\chi^{2}\right)_{j}$ is also known as the periodogram. Formally, the best-fit rotation frequency corresponds to the minimal value of $\left(\chi^{2}\right)_{j}$, but this may differ from the preferred frequency solution if the light curve is contaminated by other systematic periodic signals, if the data suffer from underestimated measurement uncertainties, or if the best-fit frequency corresponds to a dominant first harmonic (as opposed to a preferred dominant second harmonic; see Section 3.1.2).

Figure 3 details our iterative light-curve-fitting algorithm's logic. Fitting commences as long as 20 or more "reliable" data points (see Section 2.2 and Figure 2) are associated with a light curve. Irrevocably bad data points are discarded in the first round of iterations; these include detections with $7 \sigma$ or greater residuals from the initial solution. Examples of detections with such high residuals include contamination from background sources missing in the reference catalog, bad detector pixels that were not flagged by the pipeline, or spurious zero-point solutions.

In the next stage of iterations, the fit's $\chi^{2}$ per degree of freedom is reduced to $\sim 1$ (formally, it is reduced until it is less than 3; see Figure 3) by gradually inflating the observations' error bars through addition of a "cosmic error," so-named because it encompasses contamination from possible errors (in all the "cosmos"). In general, the cosmic error represents the same diverse contaminating phenomena responsible for the $>7 \sigma$ deviations seen in the initial iterations (see previous paragraph), just to a lesser extent.

Separately, this error bar inflation compensates for our model's inability to fit each asteroid's precise periodic structure using only two harmonic terms in the Fourier series. In the limit of infinite observations and sufficiently many Fourier terms, we would ideally expect our data's error bars to reflect true Gaussian variance. However, by truncating the series at two harmonics and using sufficiently precisely calibrated photometry, we are in effect choosing to sacrifice (downsample) some of our photometric precision to obtain a formally better fit at the coarser resolution limit of the model.

To illustrate use of the cosmic error, consider the example of an eclipsing binary light curve, i.e., a rotation curve that is effectively sinusoidal except for a small interval around the phase of minimum flux, when it dips to a lower-than-predicted brightness. Observations acquired during such eclipses will have systematic negative deviations greater in absolute value than would be explained by Gaussian variance alone. Increasing the error bars of these observations will decrease the fits' $\chi^{2}$ without altering the value of the fitted frequency. The fitted parameters' uncertainties (for both frequency and the linear parameters) are accordingly inflated as a penalty, and the fitted amplitude will be underestimated. As detailed in Figure 3, the initial cosmic error used is $0.002 \mathrm{mag}$, and each iteration is multiplied by a factor of 1.5 until the $\chi^{2}$ is sufficiently low. If the cosmic error exceeds $0.1 \mathrm{mag}$, the fitting is aborted. If the $\chi^{2}$ (per degree of freedom) drops below 3 while the cosmic error is still below $0.1 \mathrm{mag}$, the fitting process concludes "successfully" (see Figure 3).

Concurrently, each iteration includes a test for the presence of double peaks in the folded rotation curve (only if the fitted amplitude is at least $0.1 \mathrm{mag}$ ). In particular, if there exist two maxima and two minima in the folded light curve, we demand that the ratio of these peaks be greater than 0.2. Such a solution is preferred (see Section 3.1.2) given our ellipsoidal shape assumption, as described by Harris et al. (2014).

Denote as $f_{\text {best_global }}$ the frequency yielding the absolute minimum $\chi^{2}$ per degree of freedom value, denoted $\chi_{\min \text { _global }}^{2}$ (after the cosmic error has been tuned). If the folded light curve is single-peaked (or has only a relatively small secondary peak), then another deep minimum usually exists at the harmonic frequency $f_{\text {best_harmonic }}=0.5 \times f_{\text {best_global }}$, the local minimum $\chi^{2}$ value of which we denote $\chi_{\text {min_harmonic }}^{2}$. For cases wherein $\chi_{\text {min _harmonic }}^{2}<\chi_{\text {min _global }}^{2}+\operatorname{inv-~} \chi^{2}-\operatorname{cdf}(0.95,7)$, where inv- $\chi^{2}-\operatorname{cdf}(p, N)$ is the inverse of the $\chi^{2}$ cumulative distribution function for $N$ free parameters evaluated at $p$, then we instead choose $f_{\text {best_harmonic }}$ rather than $f_{\text {best_global }}$. The $1 \sigma$ uncertainty interval for the best-fit frequency is then found by computing the upper and lower intersections between $\chi_{\min }^{2}+\operatorname{inv}-\chi^{2}-\operatorname{cdf}(0.68,7)$ and the periodogram in the vicinity of $f_{\text {best }}$. Note that we used $n=7$ free parameters in this case, i.e., the number of elements of $X_{j}$ (Equation (18)).

\subsection{Nonlinear Phase-function Parameters}

Modeling the phase function $\phi$ with either the $G$ or $G_{12}$ model (Equations (11) and (12)) introduces a second nonlinear parameter (after the frequency $f$ ), and so we must modify the equations of the previous section accordingly. We sample $N_{\text {pha }}=200$ evenly spaced phase-function parameter values. In particular, for $G$ we test the interval $-0.3 \leqslant G \leqslant 0.7$ in steps of $\Delta G=0.005$, and for $G_{12}$ we test the interval $0 \leqslant G_{12} \leqslant 1$ in steps of $\Delta G_{12}=0.005$.

Our approach is to modify the left-hand side of Equation (16) by defining a new matrix $m_{i q}^{\prime}$ that contains all possible phasefunction-corrected observed magnitudes:

$$
m_{i q}^{\prime} \equiv m_{i}-\Phi_{i q}=\sum_{j, k} L_{i j k} X_{j k q}\left\{\begin{aligned}
i & =1,2, \ldots, N_{\mathrm{obs}} \\
j & =1,2, \ldots, N_{\mathrm{frq}} \\
k & =1,2, \ldots, N_{\mathrm{lin}} \\
q & =1,2, \ldots, N_{\mathrm{pha}}
\end{aligned}\right.
$$

where, e.g., for the case of the $G$-model (Equation (11)),

$$
\begin{aligned}
\Phi_{i q} & \equiv-2.5 \log _{10}\left[\phi\left(\alpha_{i}, G_{q}\right)\right] \\
& =-2.5 \log _{10}\left[\left(1-G_{q}\right) \phi_{1}\left(\alpha_{i}\right)+G_{q} \phi_{2}\left(\alpha_{i}\right)\right] .
\end{aligned}
$$

The linear-parameter-solution array $X$ now has an extra index $q$, reflecting the fact that we are now solving for each linear parameter as a function of the two nonlinear parameters. The design matrix has the same number of indices as before 


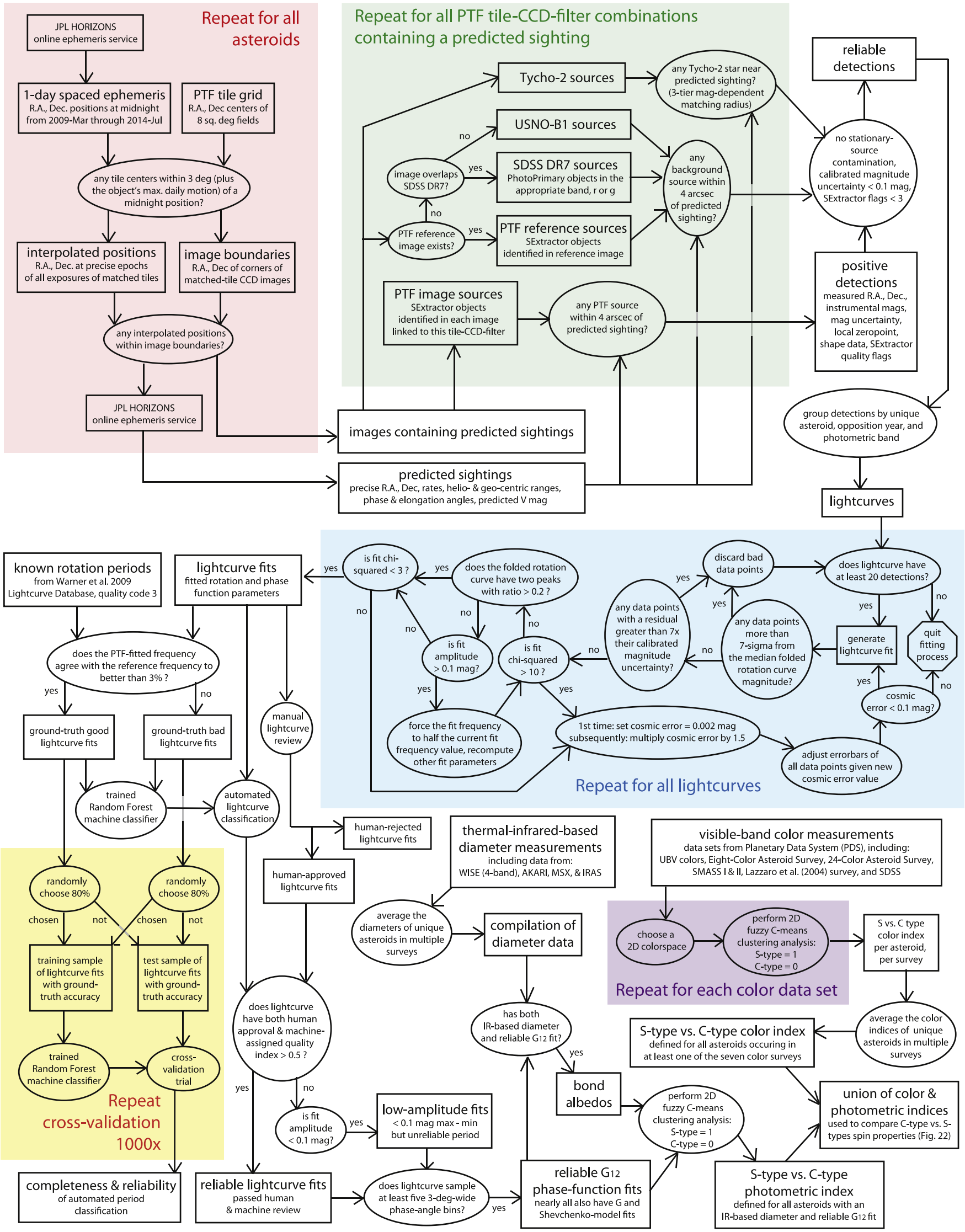

Figure 3. Diagram detailing the logic of this work's data reduction and analysis. Includes mining the survey for known-asteroid observations, aggregation of the data into light curves, vetting of the light curves, and an application wherein phase functions are compared to color-derived asteroid taxonomy. See text for details. 
(but fewer rows):

$$
L_{i j}=\left(\begin{array}{c}
1 \\
\sin \left(2 \pi f_{j} \tau_{i}\right) \\
\cos \left(2 \pi f_{j} \tau_{i}\right) \\
\sin \left(4 \pi f_{j} \tau_{i}\right) \\
\cos \left(4 \pi f_{j} \tau_{i}\right)
\end{array}\right),
$$

while the linear-parameter matrix $X$ is now

$$
X_{j q}=\left(\begin{array}{c}
H_{j q} \\
\left(A_{1,1}\right)_{j q} \\
\left(A_{2,1}\right)_{j q} \\
\left(A_{1,2}\right)_{j q} \\
\left(A_{2,2}\right)_{j q}
\end{array}\right) .
$$

The appeal in adopting the above approach is that the general solution is only slightly modified:

$$
X_{j k q}=\sum_{\ell, n, p} S_{j k \ell} L_{n j \ell}\left(B^{-1}\right)_{n p} m_{p q}^{\prime},
$$

where the only differences between Equations (19) and (29) are the $q$ indices appended to $X$ and $m$ (and the latter being redefined as $m^{\prime}$ ).

The fit's residuals $R$ are now a function of frequency and phase-function parameter:

$$
R_{i j q}=m_{i q}^{\prime}-\sum_{k} L_{i j k} X_{j k q}
$$

as is the fit's chi-squared:

$$
\left(\chi^{2}\right)_{j q}=\sum_{\ell, n} R_{\ell j q}\left(B^{-1}\right)_{\ell n} R_{n j q} .
$$

As a function of any of the linear parameters, the fit's $\chi^{2}$ varies precisely quadratically, whereas as a function of frequency it has an intricate spectral structure with many local minima. As a function of a nonlinear phase parameter $(G$ or $G_{12}$ ), the $\chi^{2}$ tends to have a single minimum (on the range we evaluate): in this sense $G$ and $G_{12}$ are more similar to the linear parameters than they are to frequency. However, the generally asymmetric shape of the phase parameter' $\chi^{2}$ dependence necessitates its grid-based numerical treatment-particularly to ensure accurate estimation of the phase parameter's uncertainty.

The two-dimensional $\chi^{2}$ surface given by Equation (31), which is defined on an $N_{\text {freq }} \times N_{\text {pha }}$ grid, can be reduced to a one-dimensional $\chi^{2}$ function by choosing, for each frequency index $j$, the phase-parameter index $q$ that minimizes the $\chi^{2}$. The result is a one-dimensional periodogram, as in Equation (24). Once the fitted frequency is identified, we compute the uncertainty in the fitted $f$ by the method described in the previous section using the $\operatorname{inv}-\chi^{2}-\operatorname{cdf}()$ function. We then likewise numerically compute the uncertainty in the phase parameter by again collapsing $\left(\chi^{2}\right)_{j q}$ to a one-dimensional vector, this time as a function of the phase parameter with the frequency fixed at the fitted value ( $j$-index), and use the inv- $\chi^{2}$ $-\operatorname{cdf}()$ function to estimate the uncertainty in the phase parameter.

As noted in Table 1, a total of 587,466 light curves exist in PTF, where each light curve by definition consists of all reliable observations of a unique asteroid observed in a single opposition in a single photometric band. Of these, only $\sim 10 \%$ (59,072 light curves) have at least 20 observations and therefore qualified for fitting with our algorithm. A total of 54,296 light curves actually produced a fit-the remaining 5000 light curves failed to produce a fit either because some observations were discarded and the total fell below 20 data points or because the fitted cosmic error grew to exceed $0.1 \mathrm{mag}$.

Figure 4 shows several examples of light curves fitted with the algorithm described in this section. In the third column (column C) of Figure 4, we show the periodograms of each light curve. Note that although the periodogram's horizontal axes are labeled with the period (for easier interpretation), the chi-squared (per degree of freedom) values are actually plotted linearly with respect to frequency. This is because, as described earlier, our sampling is uniform with respect to frequency, and the harmonics are more easily seen with constant frequency spacing. Column (D) shows the data sampling in rotational phase versus solar phase angle, a useful plot to ensure that there is no obvious correlation between the two (which could lead to an erroneous fit, e.g., for long periods, large amplitudes, and/or few data points).

\subsection{Comments on Implementation}

Each iteration in the fitting of each asteroid light curve involves evaluating the arrays and tensor-products in either Equation (19) or (29). This includes inverting the datacovariance matrix $B$ (Equation (20)) and inverting the $N_{\text {fra }}$ matrices $s_{j}$ (Equation (22)). The arrays $L, m^{\prime}, X$, and $R$ can have a relatively large number of elements, making them and their relevant products potentially taxing with respect to computational memory.

Our particular implementation of this algorithm leverages the efficient array-manipulation capabilities of MATLAB, especially its ability to perform fast matrix multiplication and matrix inversion utilizing BLAS calls ${ }^{17}$ and OpenMP multithreaded C loop code. ${ }^{18}$ Given typical numbers of observations and frequency sampling, each of our light-curve fits (including the multiple iterations) takes on average several tens of seconds to run on an eight-core machine (multithreading enabled) and typically consumes less than $\sim 4$ GB of memory using singleprecision computation.

In a tar.gz file we provide our custom MATLAB function used for fitting the $G$-parameter version of the light-curve model (asteroid_lc_fit_G.m). Analogous versions exist for the Shevchenko and $G_{12}$ models. This function takes as input an asteroid's apparent magnitudes, magnitude uncertainties, observed epochs, phase angles, and geocentric and heliocentric distances. Its outputs include the linear-parameter-solution array (Equation (28)), residuals (Equation (30)), chi-squared array (Equation (31)), and additional information about each light-curve solution such as the amplitude and peak ratios.

\footnotetext{
17 http://www.netlib.org/blas

18 http://openmp.org
} 
A



$2843[13 r] G_{12}=0.245 \pm 0.005$

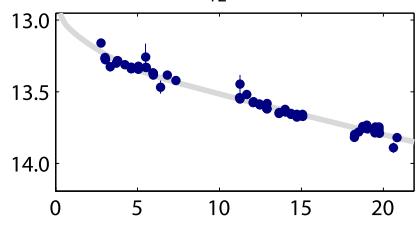

$5773[13 r] G_{12}=0.330 \pm 0.013$

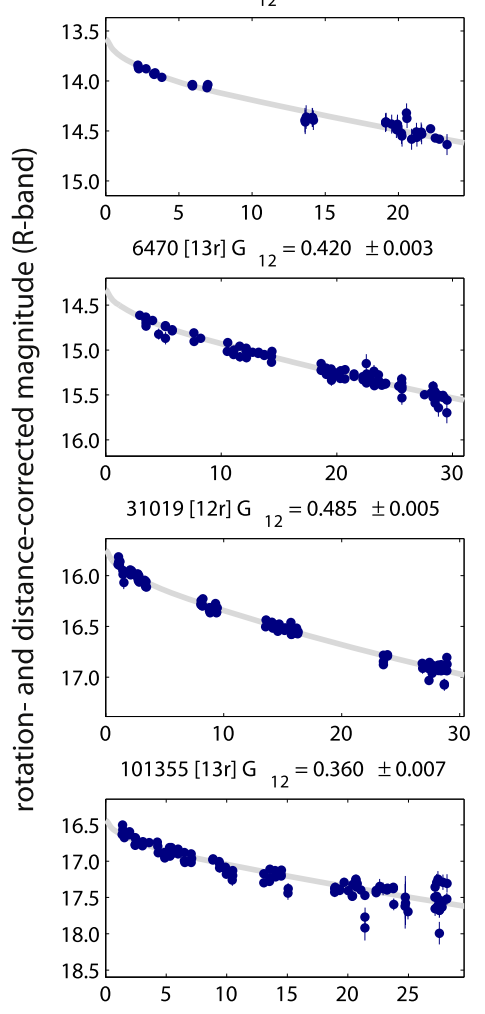

$147946[13 \mathrm{r}] \mathrm{G}_{12}=0.290 \pm 0.010$

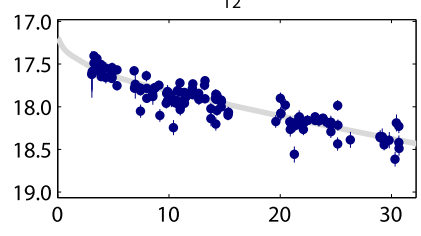

$339606[12 \mathrm{r}] \mathrm{G}_{12}=0.610 \pm 0.015$



B
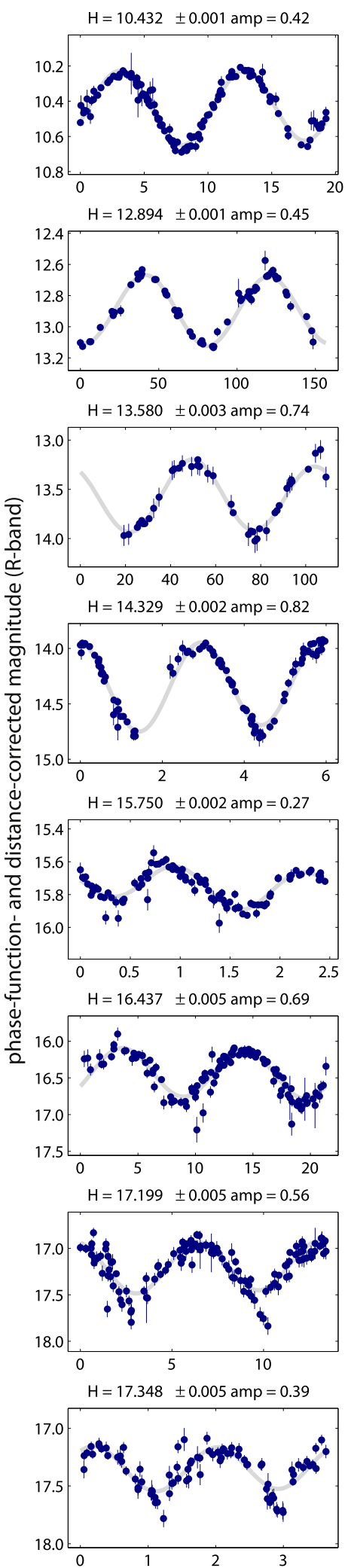

rotation-period folded time (hr)
C
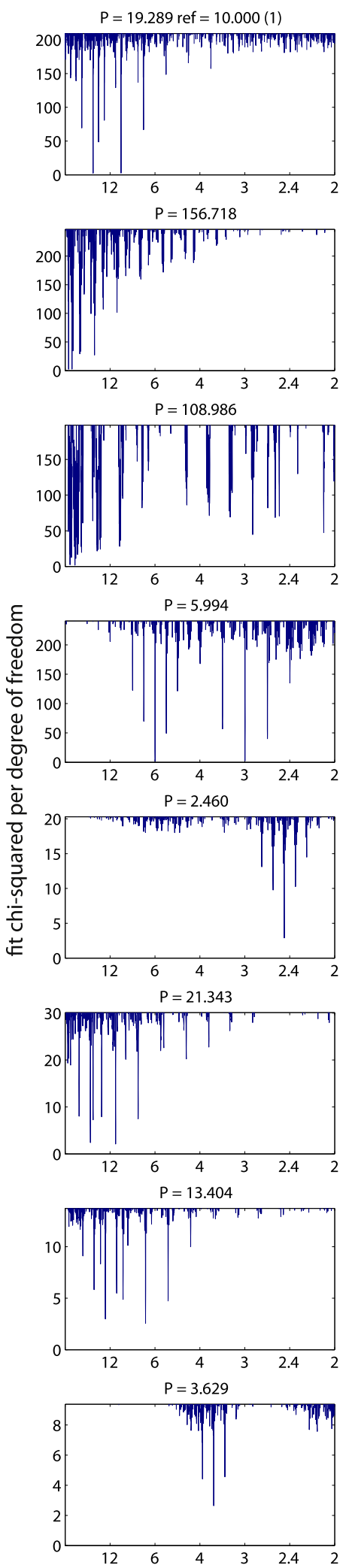

rotation period $(\mathrm{hr})$
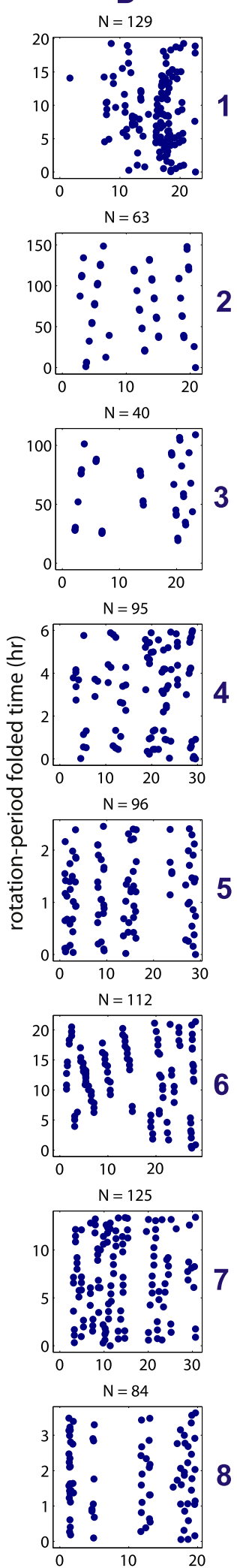

solar phase angle a (deg)

Figure 4. Examples of light curves having both well-sampled rotation and phase-function components. Each row corresponds to a different asteroid. These example asteroids are sorted vertically by their physical diameter (assuming $7 \%$ albedo); the top object is $\sim 45 \mathrm{~km}$, and the bottom object is $\sim 2 \mathrm{~km}$. Column A shows the phase curve (corrected for rotation); Column B shows the rotation curve (corrected for phase function); Column C shows the periodogram; Column D shows the distribution of the observations in rotational phase vs. solar phase angle. Above each plot is additional information depending on the column: (A) the asteroid number, followed by (in square brackets) the opposition year (most are 2013) and filter (in all cases "r") followed by the fitted $G_{12}$ parameter; (B) the fitted absolute magnitude and amplitude; (C) the fitted period (in hours); (D) the number of data points included (and shown) in the fit. 


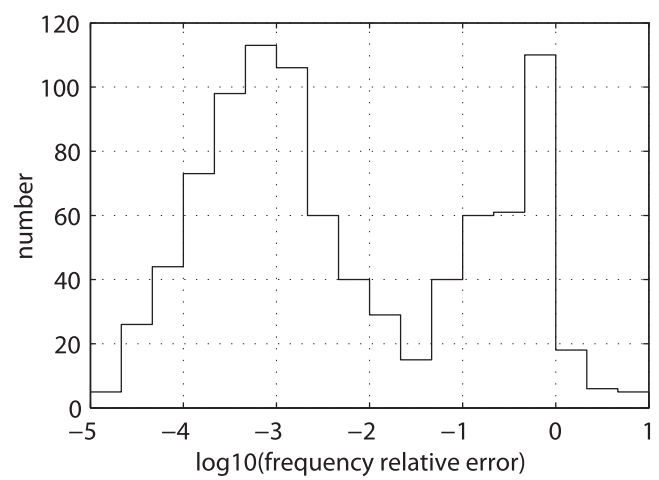

Figure 5. For the 927 light curves ( 805 unique asteroids) having a quality code 3 period in the Light Curve Database of Warner et al. (2009) and an original fit in this work, we plot the distribution of the relative error in our fitted rotation frequencies with respect to the literature-referenced frequencies. The distribution is bimodal, with the left-hand mode corresponding to those fits having better than $\sim 3 \%$ agreement.

\section{RELIABILITY OF FITTED ROTATION PERIODS}

A primary concern in the quality assessment of our fitted light-curve parameters is the validity of our derived rotation periods. In this section we describe several methods of estimating the reliability of these periods, beginning with comparison to a ground-truth subsample of known-period asteroids and followed by a full vetting of our entire sample using a combination of machine-learning and manual classification.

The fitted period may differ (slightly or significantly) between the fits using the different phase-function models. In this section for simplicity we consider only the period value obtained when fitting with the $G_{12}$ phase-function model (Section 3.2.3). In subsequent sections we will again consider all three $\phi$ models.

\subsection{Known-period Subsample}

A total of 927 ( $2 \%$ ) of our fitted light curves belong to 805 unique asteroids having a previously measured period listed in the LCDB of Warner et al. (2009). This includes only asteroids having a quality code of 3 (highest quality) in the LCDB.

Figure 5 shows that the distribution of relative errors on our fitted frequencies is bimodal, with the left mode corresponding to periods having better than $\sim 3 \%$ agreement with the reference period, and the right mode corresponding to periods in disagreement with the reference period. These disagreeing fits include light curves that differ from the reference value by a harmonic (half $=$ relative error 0.5 , double $=$ relative error 1.0 ), as well as frequencies that do not differ by a factor of two or any integer multiple. About $1 / 3$ of the light curves in Figure 5 fall into the right mode and are thus considered disagreeing fits.

Figure 6 shows some examples of these disagreeing fits. Row 1 shows an apparent low-amplitude rotator, whose fitted period of $15.7 \mathrm{hr}$ differs from the reference value of $9.7 \mathrm{hr}$. Row 2 is an object whose periodogram contains a great deal of noise, divided into two broad forests of frequency minima. The left forest appears to have been selected by our fitting algorithm, while the right forest seems associated with the true period of $\sim 2.7 \mathrm{hr}$. Row 3 contains an object whose $12 \%$ relative frequency error exceeds the $3 \%$-accuracy threshold we have defined, and so despite appearing to be a good fit it is formally categorized as inaccurate. Row 4 also looks like a reasonable fit at $6.4 \mathrm{hr}$, but disagrees with the reference period of $11.0 \mathrm{hr}$ (though the latter does have a perceptible local minimum in the periodogram). Finally, Row 5 includes a likely example of the algorithm fitting noise in the photometry of a faint asteroid.

In Figures 7 and 8 (top and middle rows) we detail the distribution of the accurately recovered-period and inaccurately recovered-period subgroups in terms of eight different lightcurve parameters. Some basic observations from these histograms are:

1. fitted periods are far less reliable if longer than $\sim 1$ day or shorter than $\sim 2.7 \mathrm{hr}$;

2. fitted amplitudes of less than 0.1 mag correspond to the least reliably fit periods;

3. light curves consisting of observations dimmer than $\sim 18.5 \mathrm{mag}$ are much less reliable than brighter light curves (though they are also far less numerous in the known-period sample);

4. fit $\chi^{2}$ (per degree of freedom) values of less than $\sim 1.7$ correlate with less reliable periods (though they are also far less numerous in the known-period sample); note that, in the fitting process, growth of the cosmic error term ceased once the $\chi^{2}$ (per degree of freedom) fell below 3 (see Figure 3);

5. the number of observations in a light curve is not directly correlated to the reliability of the fitted period;

6. the ratio of the folded light curve's two peaks, the signalto-noise ratio of the periodogram's chosen minimum, and the uncertainty in the absolute magnitude parameter are all strong indicators of the reliability of the fitted period.

The above comments reflect consideration of the onedimensional distributions in Figures 7 and 8; however, we can easily imagine that there are correlations in more dimensions not evident from these plots alone. An obvious example would be the two-dimensional distribution in amplitude versus median magnitude: reliability is presumably greater for bright asteroids having amplitudes $<0.1 \mathrm{mag}$ than it is for dim asteroids having amplitudes $<0.1 \mathrm{mag}$. Period versus amplitude is also likely an insightful distribution (and was considered, for example, by Masiero et al. 2009). The number of observations possibly would correlate with reliability if we were to restrict another parameter or parameters to some specific interval.

Rather than manually examining the period-fitting reliability as a function of all possible multi-dimensional combinations of the eight light-curve parameters detailed in Figures 7 and 8, we can take a more general approach of considering the reliability to be a single function defined on the multi-dimensional parameter space in which all of the light curves reside. We hypothesize that accurately fit light curves and inaccurately fit light curves occupy distinct regions in this multi-dimensional volume. As these volumes can overlap to some extent, we can at least estimate the probability that a light curve with that particular vector of parameters corresponds to an accurately recovered (or inaccurately recovered) period when obtained by the fitting algorithm of Section 4.

There are two general ways of accomplishing this goal. One way is to produce a large number of synthetic light curves filling out the multidimensional light-curve-parameter space, subject these synthetic light curves to our fitting algorithm, and thereby map out, e.g., by binning and interpolation, the fit reliability throughout the multi-dimensional volume. This 
A
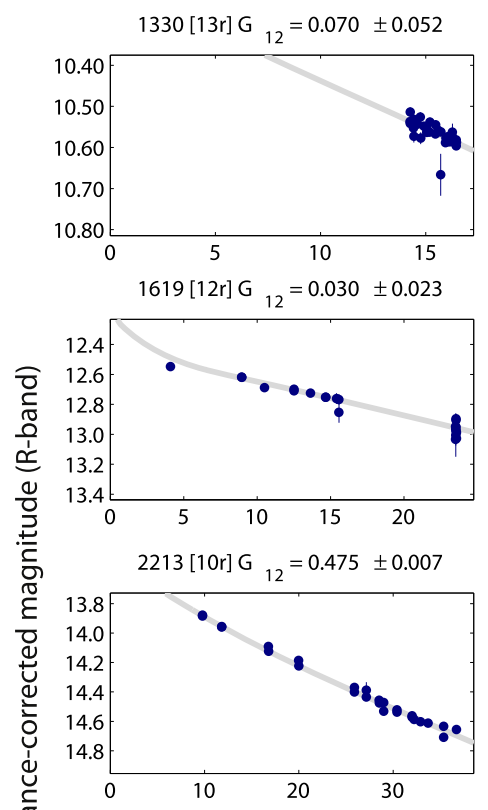

$3333[13 r] G_{12}=0.720 \pm 0.010$
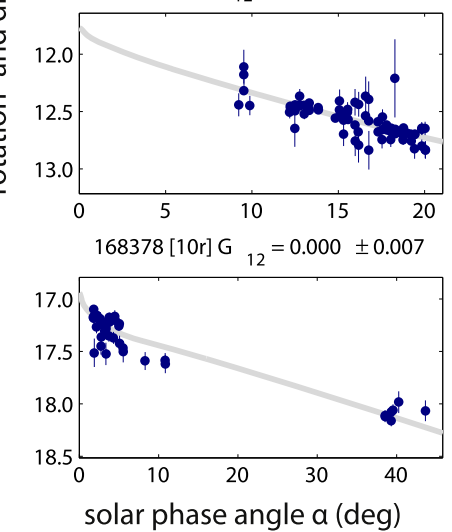

B


悹 13

동
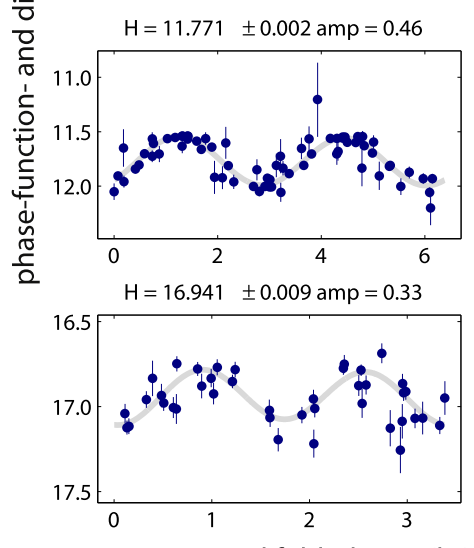

rotation-period folded time (hr)
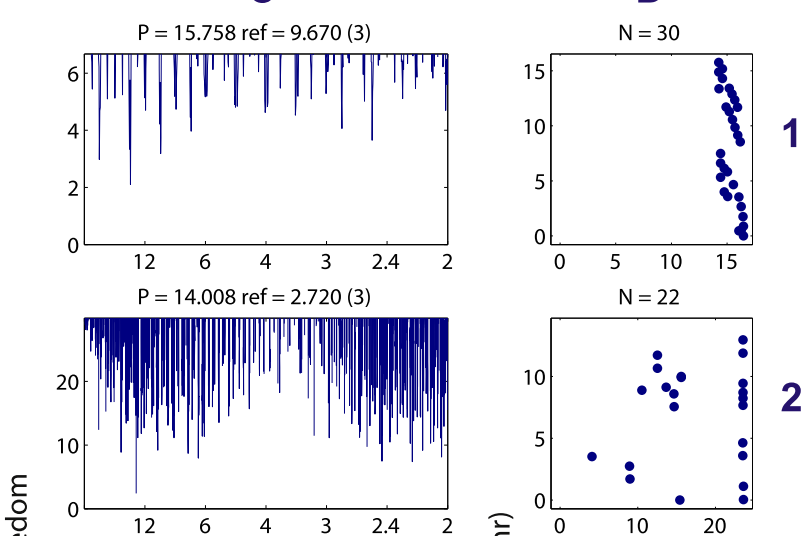

1

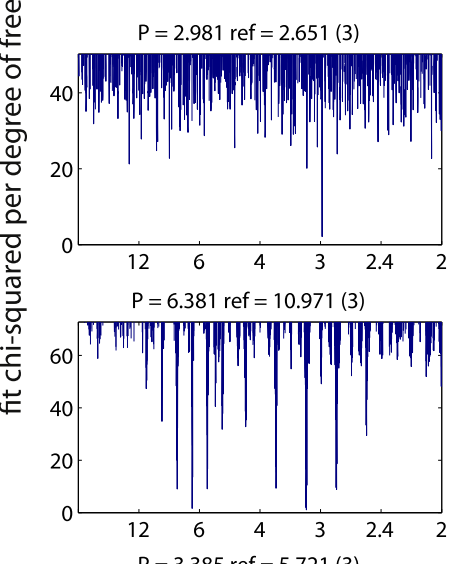

$\mathrm{P}=3.385$ ref $=5.721$ (3)

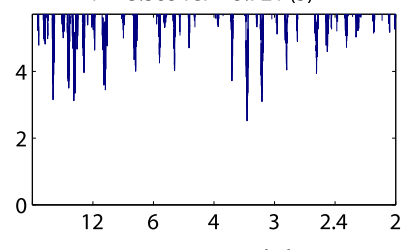

rotation period $(\mathrm{hr})$



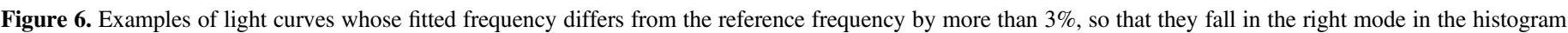

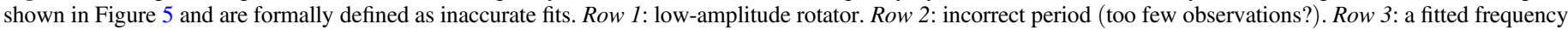

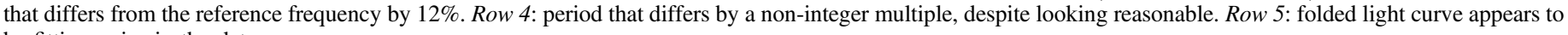
be fitting noise in the data.

method requires us to accurately simulate all sorts of varying sampling cadence as well as measurement uncertainties, including contributions from both systematics and noise, and it requires significant extra computing time to actually subject the synthetic data to our fitting procedure. The second method - the approach we take in this work-uses a ground-truth sample (the known-period light curves already described in this section) to train a machine classifier to discriminate reliable versus unreliable fits within the multi-dimensional light-curveparameter space.

\subsection{Machine Learning}

We adopt a supervised ensemble-method approach for classification, originally popularized by Breiman et al. (1984), specifically the random forest (RF) method (Breiman 2001). RF classification has extensive and diverse applications in many fields (e.g., economics, bioinformatics, sociology). Within astronomy in particular RF classification is one of the more widely employed methods of machine learning, though many alternatives exist. For example, Masci et al. (2014) use the RF method for variable-star light-curve classification, while others have approached this problem via the use of, e.g., support vector machines (Woźniak et al. 2004), Kohonen self-organizing maps (Brett et al. 2004), Bayesian networks and mixture-models (Mahabal et al. 2008), principle component analysis (Deb \& Singh 2009), multivariate Bayesian and Gaussian mixture models (Blomme et al. 2011), and thick-pen transform methods (Park et al. 2013).

For general descriptions of RF training and classification, we refer the reader to Breiman (2001), Breiman \& Cutler (2004), and the many references cited by Masci et al. (2014). Our use of an RF classifier is particularly motivated by its alreadyproven application to the discovery and classification of astrophysical transients in the same PTF survey data (Bloom et al. 2012), as well as streaking near-Earth asteroid discovery in PTF data (A. Waszczak et al. 2015, in preparation).

Machine-learning application generally consists of three stages: training, cross-validation, and classification. In the 

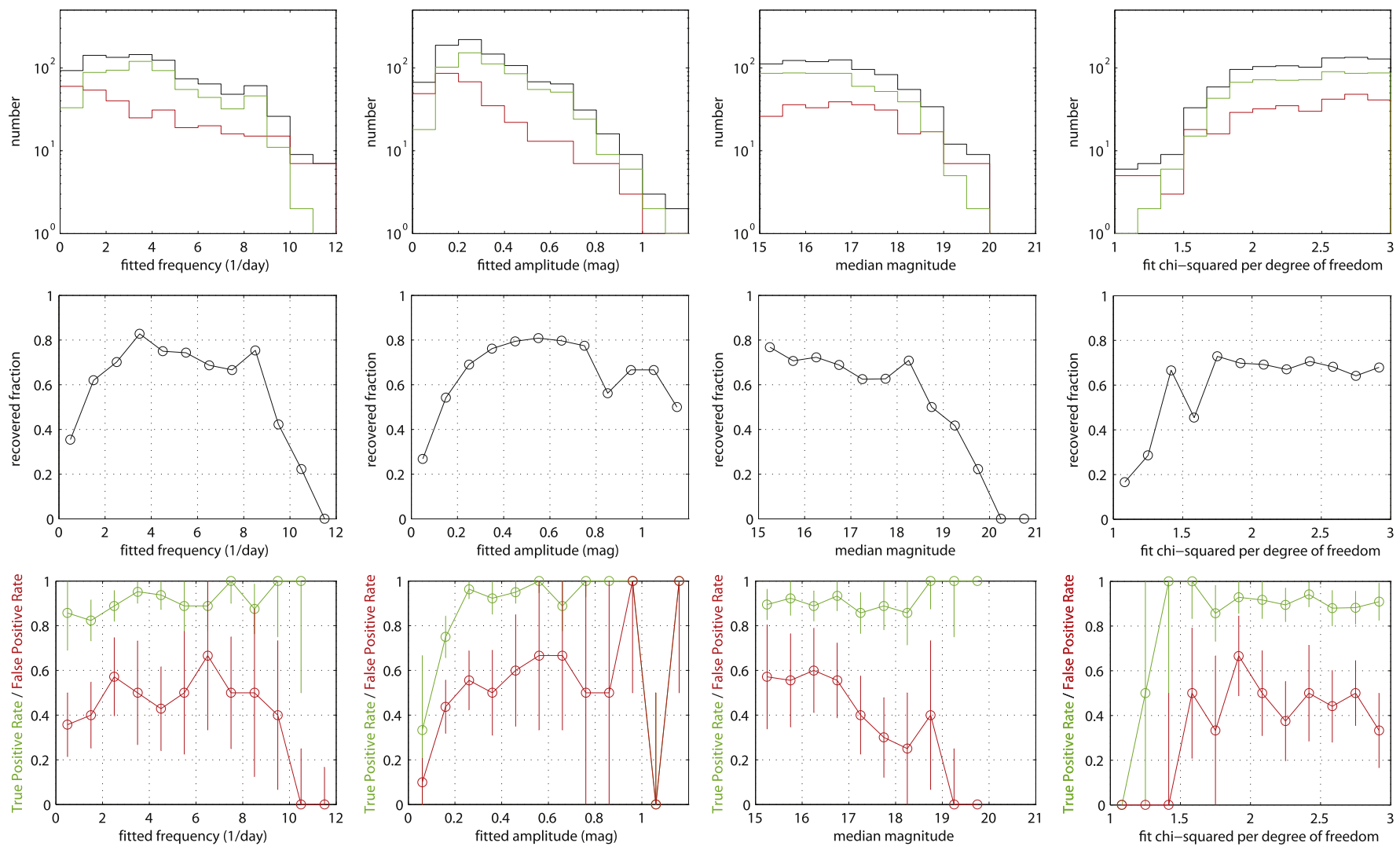

Figure 7. Top row: 927-light-curve known-period sample (black), divided into the accurately fitted (green) and inaccurately fitted (red) subgroups. Middle row: ratio of the green to black histograms. Bottom row: results of cross-validation of the machine classifier (see Section 5.2.2).
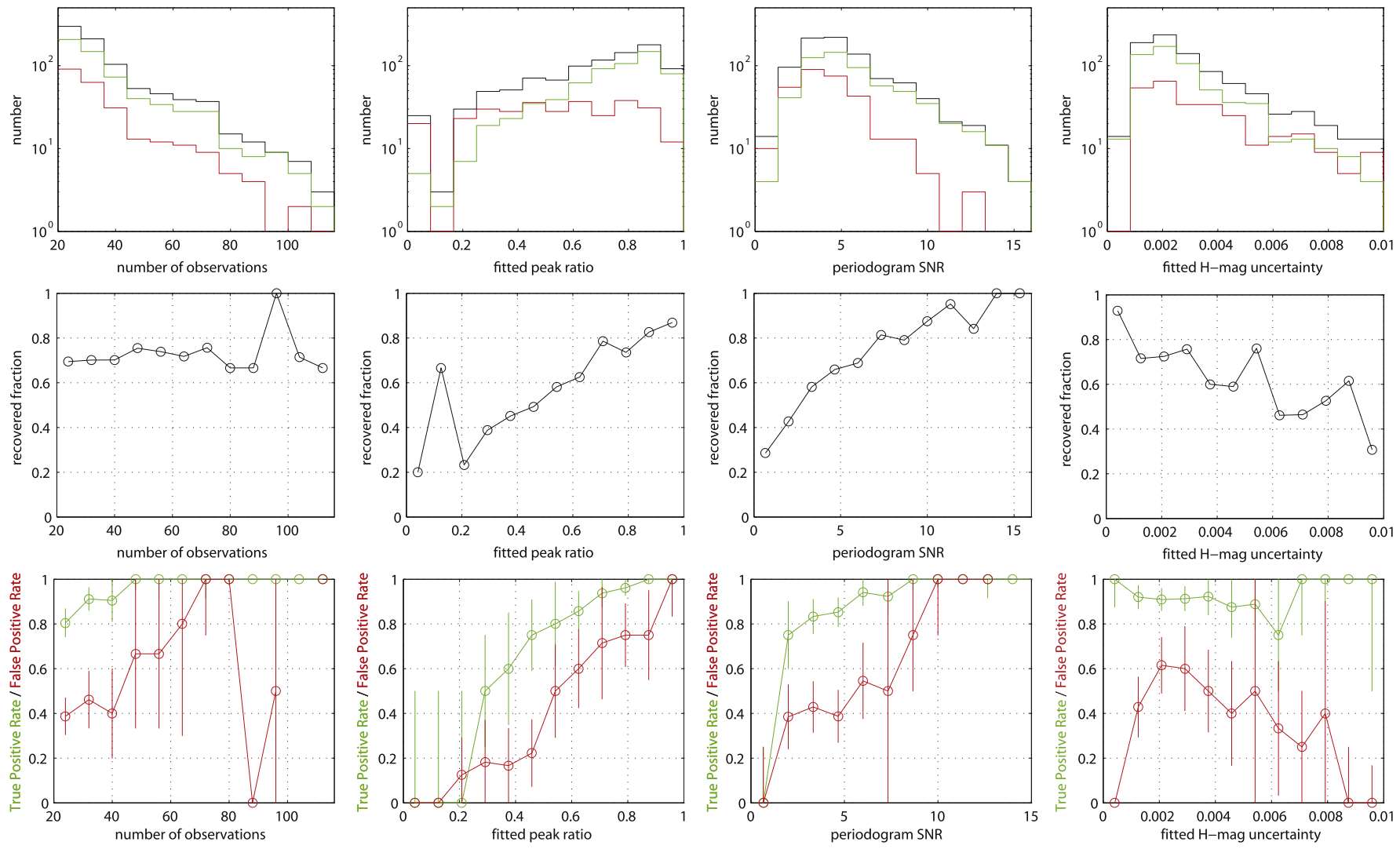

Figure 8. Top row: 927-light-curve known-period sample (black), divided into the accurately fitted (green) and inaccurately fitted (red) subgroups. Middle row: ratio of the green to black histograms. Bottom row: results of cross-validation of the machine classifier (see Section 5.2.2). 
training stage of building a machine classifier, the multidimensional parameter space is hierarchically divided into subspaces called nodes; these nodes collectively comprise a decision tree. The smallest node-also known as a leaf-is simply an individual data point (in our case, a single light curve). Given a set of leaves with class labels, one can build an ensemble of trees (called a forest), each tree representing a unique partitioning of the feature space, wherein the nodes are split with respect to different randomly chosen subsets of the parameter list. Each node splitting attempts to maximize the separation of classes between the sub-nodes. Serving as a model, in the subsequent classification stage the forest allows one to assign a probability that a given vector of features belongs to a given class. During cross-validation (an essential early stage in this process), the training and classification steps are repeated many times, each time using different subsamples (of labeled data) as the training data and testing data. Crossvalidation evaluates the classifier's performance and ensures that it is not overfitting the training data.

For our light curves, we are interested in a binary classification, i.e., whether the fitted period is accurate ("real") or inaccurate ("bogus"). Bloom et al. (2012) coined the term realBogus to describe this binary classification probability in the context of extragalactic transient identification. In the present work we are essentially adapting Bloom et al.'s realBogus concept to the problem of light-curve-period reliability assessment.

We employ a MATLAB-based Random Forest classifier, ${ }^{19}$ which is a port of the original RF software (originally implemented in $R$ ). This software includes two main functions, which perform the training and classification steps separately.

\subsubsection{Classifier Training}

Our training data consist of the known-period light curves (see the previous section) belonging to the two classes under consideration: 618 light curves having accurately fit rotation periods and 309 light curves having inaccurately fit periods. Membership in one class versus the other depends on our arguably arbitrary $3 \%$ relative accuracy threshold, though we claim that the clearly bimodal shape of the distribution in Figure 5 justifies this $3 \%$ criterion. We note also that the classifier ultimately only provides a probability that a given light curve belongs to one class or the other, so that objects very near to the $3 \%$ cutoff may conceivably correspond to classification probabilities close to 0.5 .

An important point is that the "ground-truth" reference periods we have taken from the database of Warner et al. (2009) may include some number of inaccurate periods. Such periods may be the product of erroneous fitting on the part of any one of its many different contributors, each of whom may employ a different fitting procedure and/or adhere to different confidence criteria. For the sake of this work, however, we consider all quality code 3 periods to be accurate-any actual inaccuracy will contribute to decreased classifier performance.

Besides ground-truth periods that are simply inaccurate, we also, in principle, risk contamination from reference periods that are no longer accurate. We assume that the majority of asteroids' periods are not changing with time, at least not at levels measureable with our data. For instance, direct measurement of the YORP mechanism in at least one asteroid

\footnotetext{
19 https://code.google.com/p/randomforest-matlab
}

(Lowry et al. 2007) reveals a relative rotation period change of several parts per million over several years. Any measureable period changes would likely be due to recent collisional events. The case of asteroid 596 Scheila (Bodewitts et al. 2011) demonstrates that detectable collisional events among mainbelt asteroids do occur on a relatively regular basis, though even this robustly detected collision imparted no measurable change in the asteroid's spin rate (Shevchenko et al. 2013).

Although Figures 7 and 8 detail the period-fitting reliability as a function of only eight light-curve parameters, we construct our classifier using 12 additional parameters, for a total of 20 light-curve parameters. In the context of machine learning these parameters are known as features. The 20 features we use were chosen on the basis of their availability (most are output directly by the fitting process and do not require additional computation), as well as their actual importance (as computed during the cross-validation tests described in the next section).

Figure 9 visualizes the two-dimensional correlation coefficients for all possible pairs of the 20 light-curve features. Overall, the correlation structure of the training sample qualitatively resembles that of the full data set, implying that the training set fairly well represents the overall data set in terms of its feature-space structure. On the other hand, the distributions (e.g., median value, range of values) of individual features in the training set do not necessarily match the distributions in the full data set. An obvious example is that the full data set contains far more faint asteroids than does the training sample, even though in both cases the median magnitude (medMag) is positively correlated with quantities like rmsfit (due to Poisson noise) and hMagRef (since larger asteroids tend to be brighter).

Our 20 light-curve features are listed in Table 2, in order of decreasing importance. Most of these quantities we have discussed already in previous sections in the context of our model and fitting procedure. The list also includes two features characterizing the magnitude distribution of the folded light curve: (1) Stetson's $K$-index, a measure of the kurtosis borrowed from variable-star-light curve analysis (Stetson 1996), and (2) a "cusp index" that quantifies the extent to which the dimmest $10 \%$ of the data points in the folded light curve deviate from the best fit relative to the other $90 \%$ of the data points. We designed the cusp index to potentially identify eclipsing systems that are poorly fit by the two-term Fourier approximation but nonetheless may have accurately fit periods (examples of light curves with such cusp-like minima appear in Figure 10). Eclipsing binaries would be most properly treated with a different model entirely, as would tumbling asteroids (which we also did not systematically try to identify in the data, and probably lack reliable light curve solutions when subjected to this work's algorithm).

\subsubsection{Classifier Cross-validation}

To ascertain the trained classifier's capabilities, and to ensure that the classifier is not overfitting the training data, we perform a series of 1000 cross-validation trials. In each trial we split each class (accurate fits and inaccurate fits) into a training subsample (a randomly chosen ${ }^{20} 80 \%$ of the class) and a test subsample (the remaining $20 \%$ of the class). We then

\footnotetext{
20 Another standard, slightly different approach is to evenly split the training data into $k$ disjoint sets (a process called $k$-folding). Also, our choice to separately partition the two classes into training and test subsamples could be omitted.
} 
Table 2

Summary of the 20 Light-curve Parameters (Features) Used by Our Period-Quality Classifier

\begin{tabular}{|c|c|c|}
\hline Feature & Importance $(\%)$ & Description \\
\hline peakRatio & 11.1 & Ratio of the fitted light curve's two peaks $(=\max -\min )$. Zero if only one peak, one if exactly the same height. \\
\hline amplitude & 10.2 & Fitted amplitude of the folded light curve. Equivalent to the height ( $\max -\min )$ of the larger of the two peaks. \\
\hline periodFit & 8.6 & Rotation period value obtained using this work's data and fitting algorithm. \\
\hline hMagErr & 5.8 & Uncertainty in the fitted $H$-magnitude (i.e., error in the fitted absolute magnitude) \\
\hline a12Coeff & 4.3 & Fourier coefficient $A_{12}$ \\
\hline medMag & 4.1 & Median calibrated magnitude (in the photometric band specific to the light curve, either $R$ or $g$ ) \\
\hline chisq & 4.1 & Reduced chi-squared of the fit, i.e., $\chi^{2}$ per degree of freedom) \\
\hline a21Coeff & 4.0 & Fourier coefficient $A_{21}$ \\
\hline a11Coeff & 3.8 & Fourier coefficient $A_{11}$ \\
\hline rmsFit & 3.8 & Root-mean-squared residual of the fit \\
\hline cuspIndex & 3.6 & "Cusp index": Median squared residual of the dimmest $10 \%$ points divided by the median squared residual of all other point \\
\hline numObsRem & 2.9 & Number of observations removed during the fitting process (due to $>7 \sigma$ residuals with respect to preliminary fits) \\
\hline CosmicErr & 2.1 & Final "cosmic error" value at end of fitting process $(<0.1 \mathrm{mag}$ in all cases) \\
\hline
\end{tabular}

Note. See text for a discussion of the cross-validation-derived importance value (Section 5.2.2).
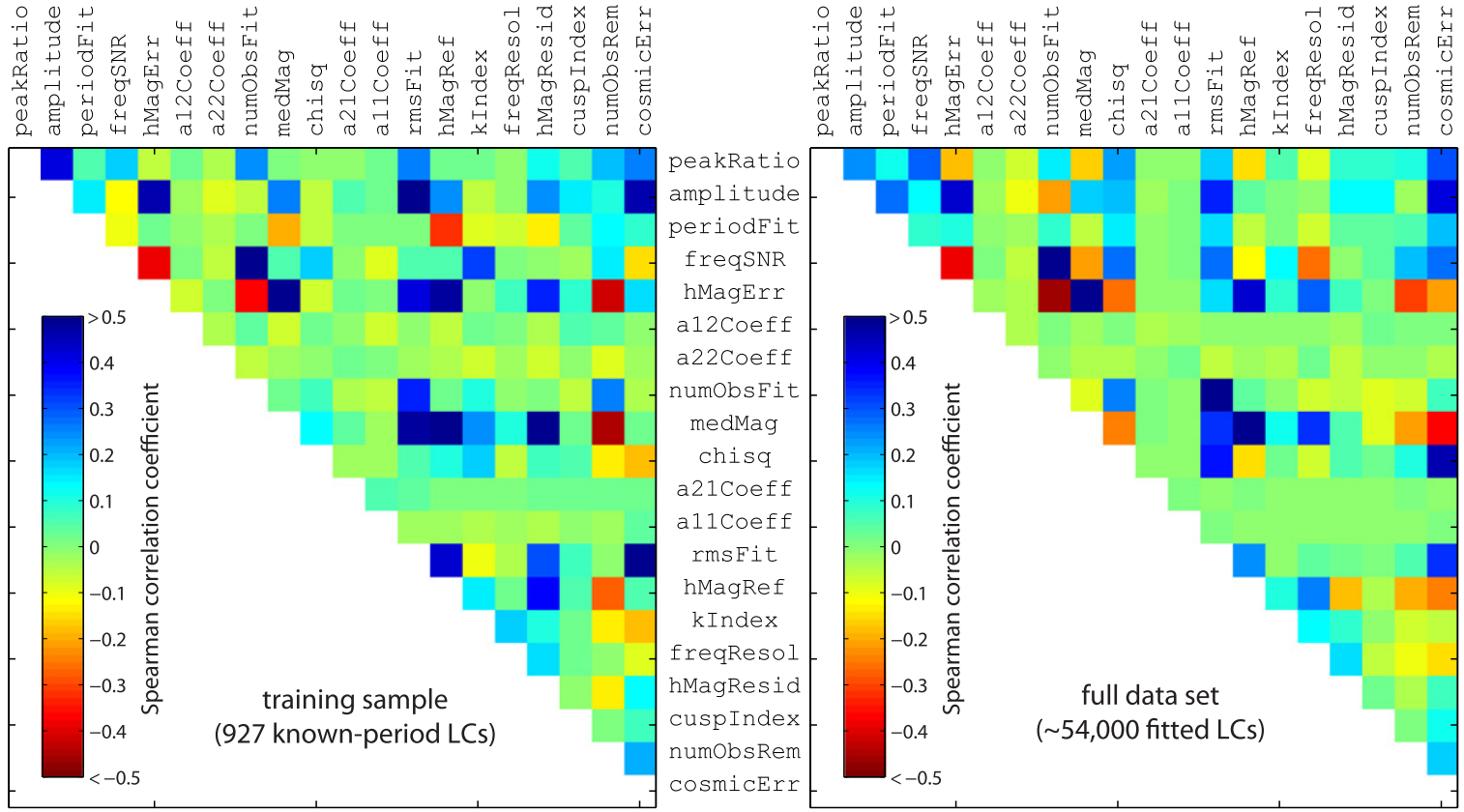

Figure 9. Correlation matrices (Spearman's $\rho$ coefficient) for the 20 light-curve features (Table 2) in the training sample (left) and in the full data set (right).

train a classifier using the combined training subsamples and subsequently employ the classifier on the combined test subsamples. In each of the trials, the classifier outputs a classification probability (score) for each object in the test sample, and we track the TPR (fraction of accurate period fits that are correctly classified above some threshold probability) as a function of the FPR (fraction of inaccurate period fits that are incorrectly classified above said threshold probability). See Figure 11 for a summary of these terms.

The results of the cross-validation are shown in Figure 12. By tuning the minimum classification probability used to threshold the classifier's output, one effectively moves along the hyperbola-shaped locus of points in TPR-versus-FPR space seen in the plot. Several points have labels $(p=\ldots)$ indicating the corresponding threshold probability (adjacent points being separated by $\Delta p=0.05$ ). The error bars in Figure 12 represent the standard deviation of the location of each point over all 1000 trials, while the point centers are the average locations.

A classification threshold of $p>0.5$ is conventionally used when quoting single FPRs and TPRs. In our case, this gives $\mathrm{FPR}=0.45 \pm 0.07$ with $\mathrm{TPR}=0.89 \pm 0.03$. The contamina tion of positively classified light curves in the cross-validation 
A



B

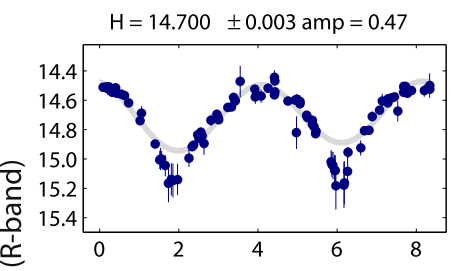

$\frac{0}{0} \quad H=15.192 \pm 0.004 \mathrm{amp}=0.93$
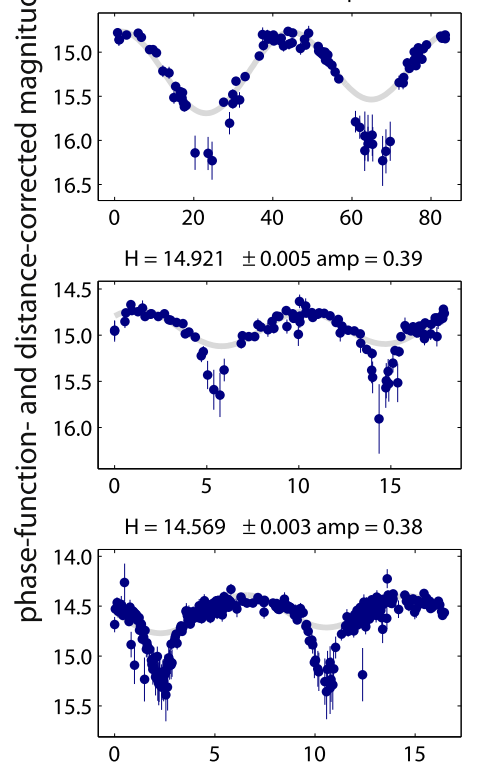

rotation-period folded time (hr)
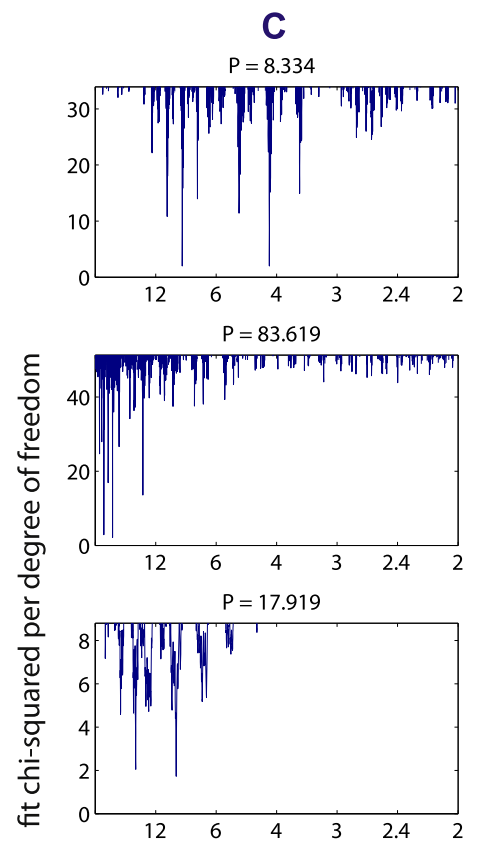

$P=16.504$ ref $=16.540$ (3)

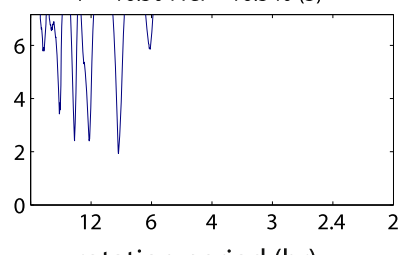

rotation period $(\mathrm{hr})$


solar phase angle a (deg)

Figure 10. Examples of reliable light curves whose folded rotation curves include cusp-like minima (systematic negative deviation from the second-order Fourier fit at minimum brightness), suggestive of a binary system. Many more examples exist in our light curves; however, in this work we have not specifically flagged such light curves. Future works will more carefully label and analyze this particular class of objects.

\begin{tabular}{|c|c|c|c|}
\cline { 3 - 4 } \multicolumn{2}{c|}{} & \multicolumn{2}{c|}{ classified (predicted) as: } \\
\cline { 3 - 4 } $\begin{array}{c}\text { actual } \\
\text { accurate } \\
\text { period } \\
\text { accuracy }\end{array}$ & $\begin{array}{c}\text { accurate } \\
\text { period } \\
\text { tround) }\end{array}$ & $\begin{array}{c}\text { true } \\
\text { positive } \\
\text { (TP) } \\
\text { period }\end{array}$ & $\begin{array}{c}\text { false } \\
\text { negative } \\
\text { (FN) }\end{array}$ \\
\cline { 2 - 4 } & $\begin{array}{c}\text { inaccurate } \\
\text { period }\end{array}$ & $\begin{array}{c}\text { false } \\
\text { positive } \\
\text { (FP) }\end{array}$ & $\begin{array}{c}\text { true } \\
\text { negative } \\
\text { (TN) }\end{array}$ \\
\hline \multirow{T}{*}{ TPR $=\frac{\text { TP }}{\mathrm{TP}+\mathrm{FN}}$} & FPR $=\frac{\text { FP }}{\mathrm{FP}+\mathrm{TN}}$
\end{tabular}

Figure 11. Definitions of true vs. false and positive vs. negative labels. TPR is sometimes called the completeness or sensitivity, while FPR is otherwise known as the false-alarm rate, one minus the reliability, or one minus the specificity.

trials depends also on the actual class ratios in the sample being classified. In particular, since $\sim 1 / 3$ of our known-period light curves are inaccurate fits (Figure 5), it follows that among all light curves the classifier labels as accurate fits, the contaminated fraction is $(0.45 \times 1 / 3) /(0.89 \times 2 / 3+0.45 \times$ $1 / 3) \approx 1 / 5$. If instead of using the classifier we just randomly labeled some fraction of the light curves as accurate and the rest as inaccurate, the resulting contamination would be $1 / 3$ (i.e., worse than the $1 / 5$ afforded by the classifier, as expected).

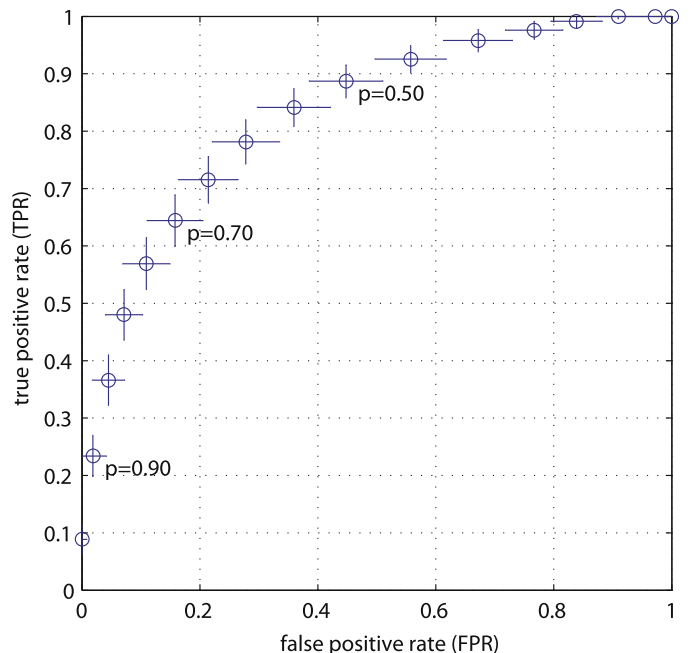

Figure 12. TPRs vs. FPRs for the cross-validation trials. Such as plot is sometimes referred to as a receiver operating characteristic (ROC) curve. Each trial trains the classifier using a randomly chosen $80 \%$ of the known accurate fits and $80 \%$ of the known inaccurate fits among the 927 light curves that have reference periods. The $20 \%$ remaining light curves serve as the test sample. Moving along the hyperbolic locus of points in this plot is equivalent to tuning the classification probability threshold from zero (lower left of the plot) to one (upper right of the plot). The error bars represent the scatter in the 1000 cross-validation trials. 

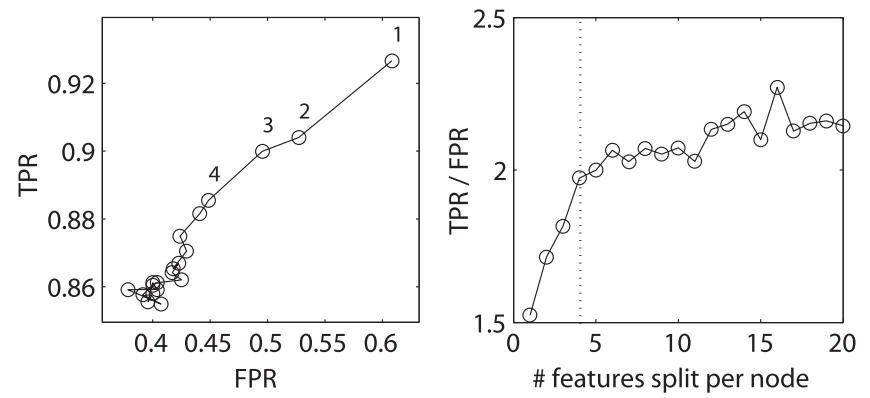

Figure 13. Varying the number of features that are randomly split per node in the decision-tree-building process affects both the TPR and FPR. The values plotted here correspond to the $p>0.5$ classification threshold; each point was generated by the exact same process for which the results in Figure 12 were generated, only varying the number of features with respect to which nodes are split. In the left plot, the first four points are labeled with the number of features for that trial (for $n>4$ we omit the label). In our actual implemented model (Figure 12) we chose $n=4$ features, the value after which the TPR/FPR ratio plateaus at approximately 2, and also the value Breiman (2001) recommends, i.e., the square root of the total number of features (in our case, $\sqrt{20} \approx 4$ ).

Several parameters can be adjusted or tuned when training an RF classifier. First is the number of decision trees generated during the training stage. Classification accuracy typically increases with the number of trees and eventually plateaus. Most applications employ hundreds to thousands of trees; we here use 1000 trees. Another tunable parameter is the number of randomly selected features (out of the 20 total here considered) with respect to which nodes are split in building the decision trees. Breiman (2001) recommends using the square root of the number of features. We ran the cross-validation for all possible numbers of features with respect to which the nodes can be split (i.e., all numbers between 1 and 20). The results are in Figure 13. We chose $n=4$ as the number of features to split, both because the classifier's performance plateaus after that value and because it follow's the recommendation of Breiman $(2001)(4 \approx \sqrt{20})$ features.

Other parameters that can be tweaked are the maximum depth of a tree, the minimum number of samples per leaf, the minimum number of samples used in a split, and the maximum number of leaf nodes. We do not constrain any of these parameters, meaning we allow trees of any depth, with any number of leaf nodes, leaf nodes consisting of a single sample, and splits based on the minimum of two samples. We note that as a result our model optimization is not comprehensive and it is possible that a better classifier could be trained. However, the relatively small training sample size here is likely the limiting factor; additional data are necessary to substantially improve the classifier performance.

In the bottom rows of Figures 7 and 8, we detail the dependence of the TPR and FPR on various light-curve parameters. Averaging (marginalizing) over any of the $x$-axis quantities in these bottom-row plots (while also weighting each bin by the number of light curves it contains; see the top row of plots in Figures 7 and 8) produces precisely the TPR and FPR values of the $p=0.5$ data point in Figure 12 .

In addition to the TPR and FPR estimates, cross-validation allows us to quantify the relative importance of the features by computing the average depth in the trees at which a split was performed with respect to each feature. Those features with respect to which the training sample is consistently divided early in the building of each tree are deemed more important (i.e., more discriminating) than those features that are split later, as the tree-building process tries to maximize the separation of the classes as early as possible by splitting features in an optimal sequence. Both Table 2 and Figure 9 list the features in order of importance.

Note that we had manually guessed several of the most important features-namely, peakRatio, freqSNR, and hMagErr-prior to any machine-learning work via inspection of the plots in Figure 8. The numerical importance values thus agree with these initial observations and also quantify the significance of features that would be difficult to ascertain manually. For instance, numObsFit appears (in Figure 8) not to be related to the fitting accuracy, while medMag (Figure 7) does appear related to accuracy (fainter light curves being less accurate), yet these two features evidently have equal importance in the classification process (see Table 2). Figure 9 indicates that numObsFit and medMag have quite different correlation relationships with respect to more important features. Hence, it would not be surprising if their onedimensional distributions (in Figures 7 and 8) bear no resemblance to the multi-dimensional distributions on which the decision trees are defined and in which these two parameters apparently carry comparable weight.

\subsubsection{Machine-vetted Light Curves}

Having trained the machine classifier as described in Section 5.2.1, we use it to predict the validity of our remaining $\sim 53,000$ fitted periods (of $\sim 48,000$ unique asteroids) that lack quality code 3 reference periods in Warner et al. (2009). The automated classifier assigned positive reliability scores $(p \geqslant 0.5)$ to 19,112 of the light curves $(35 \%$ of the total data set). Figure 14 details the distribution of the light curves (rawfitted, machine-vetted, and other subsets) with respect to some of the most important light-curve features.

With respect to rotation period (Figure 14 panel (A)), the classifier rejects the largest fractions of light curves in the long-period ( $\gtrsim 1$ days) and short-period $(\lesssim 2.7 \mathrm{hr})$ bins. From Figure 7 (bottom row, leftmost column), we know that the classifier's completeness does not drop significantly for these long- and short-period objects, nor is the FPR higher among them. Hence, we have reason to trust the classifier's heavy rejection of periods in these bins, and therefore we conclude that our fitting algorithm (Section 4) is prone to erroneously fitting periods in these period extremes (as was also suggested in the known-period sample in Figure 7).

Panel (C) shows that the mode of the apparent-magnitude (medMag) distribution for machine-approved light curves is $\sim 19 \mathrm{mag}$, as compared to the predominantly $V \lesssim 17 \mathrm{mag}$ known-period training sample. Comparing this to Figure 2 panel A shows that the limiting magnitude of reliable light curves is comparable to that of individual detections.

Panel (E) of Figure 14 shows that the raw output of our fitting process contains peak-ratio values that are uniformly distributed above 0.2 , this particular value being a hardcoded threshold that double-peaked light curves (at least those with amplitudes $>0.1 \mathrm{mag}$ ) output by our fitting algorithm must satisfy (see Figure 3 and Section 4.1). The classifier's output clearly indicates that reliability is linearly related to the peak ratio, as was also prominently seen in Figure 8. Because Figure 8 also indicates that the classifier's TPRs and FPRs also relate linearly with peakRatio, we conclude that 

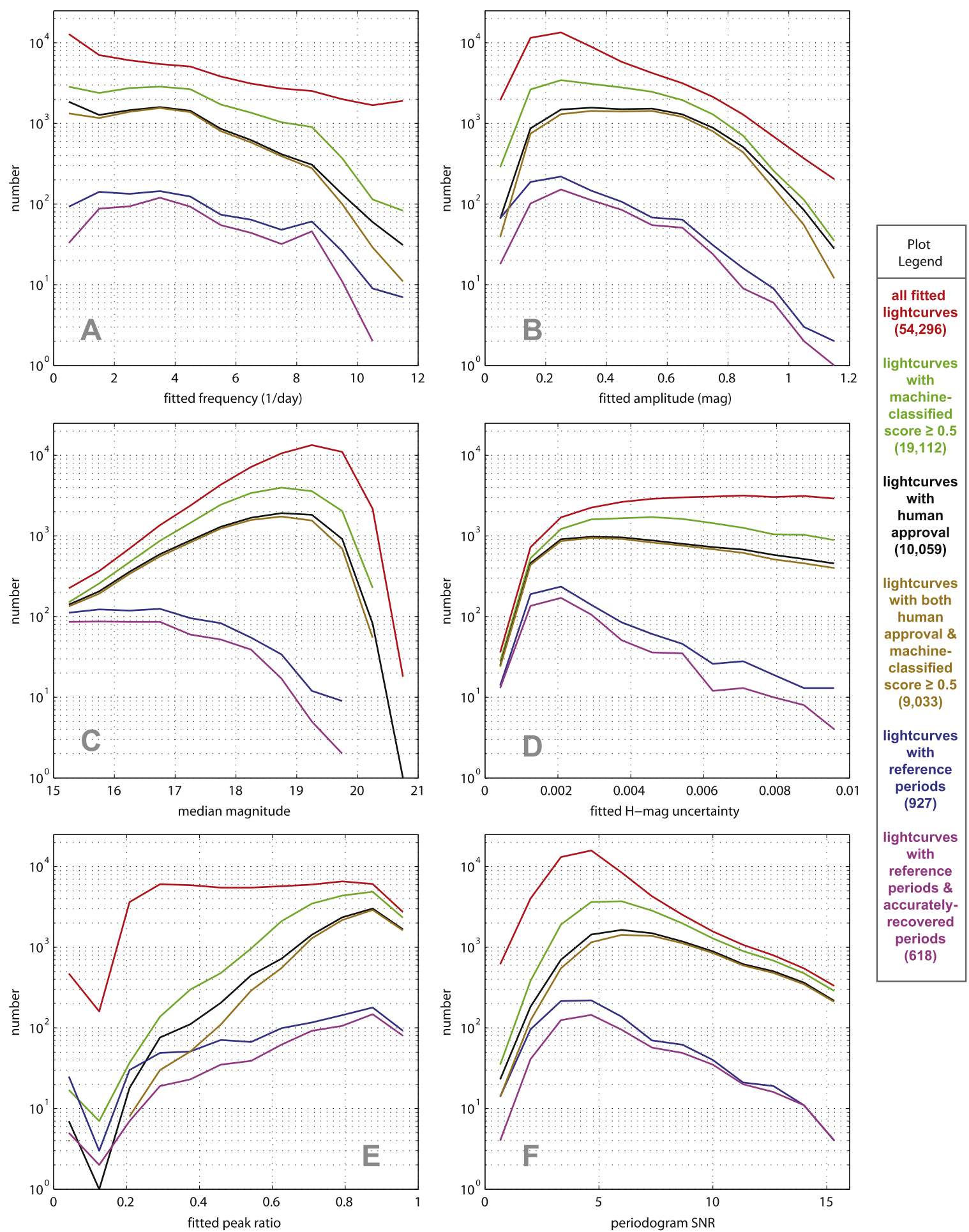

Figure 14. Distributions of PTF-fitted light curves (and various subsets thereof) in select features/parameters. These plots are histograms with the same binning as the top rows of Figures 7 and 8. For better readability we here use line-connected bin points (rather than the stair-plot format used in, e.g., Figure 5).

the slope of the peakRatio distribution for the machinevetted light curves is likely an upper limit for the true slope.

\subsection{Manual Screening}

In addition to machine-based vetting, we manually inspected all 54,296 of the light curves that were output by our fitting process. A human screener first studies the ground-truth known-period examples (Section 5.1) in an effort to learn to distinguish between accurate and inaccurate fits. Only the $G_{12}$ fit is considered (as was the case with the automated classifier), and for each light curve the screener inspects precisely the amount of information included, for example, in Figures 4, 6, and 10 of this paper. Specifically, for each light curve the screener views a row of four plots: (1) the rotation-corrected phase curve, (2) the phase-function-corrected folded rotation curve, (3) the periodogram, i.e., the reduced $\chi^{2}$ plotted linearly against frequency (labeled however with the corresponding 
A



B

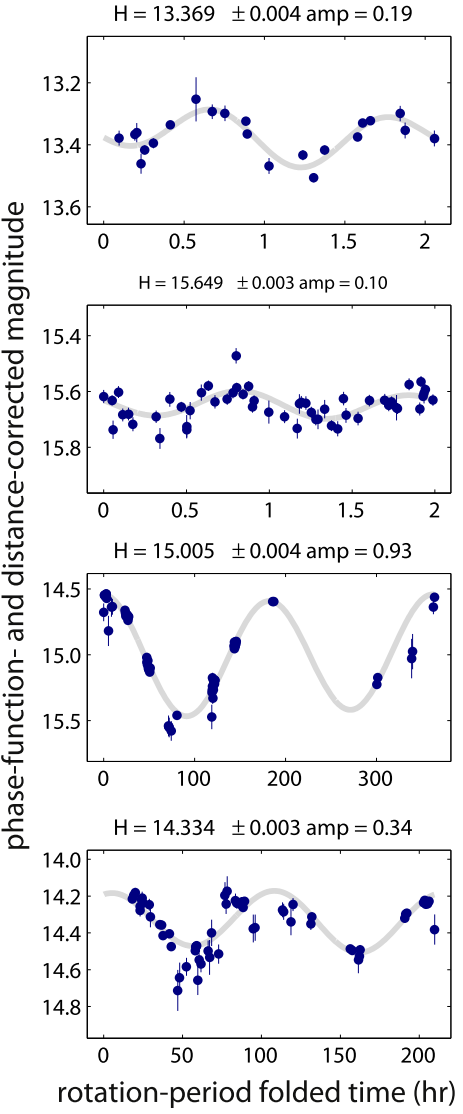

C



D
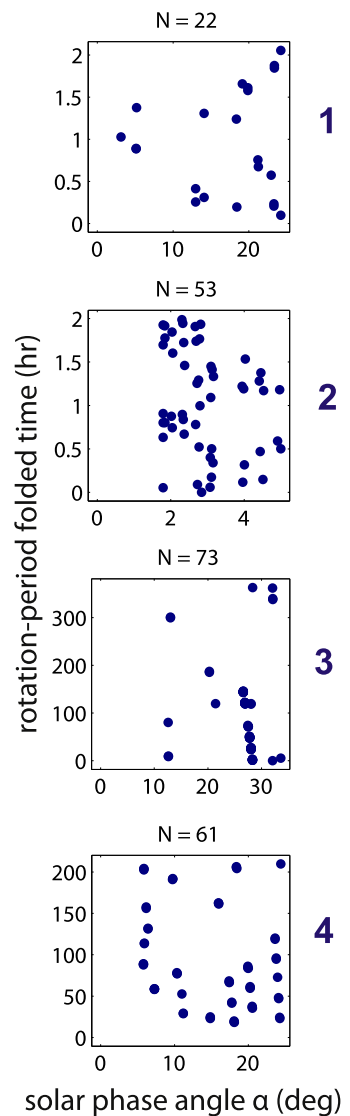

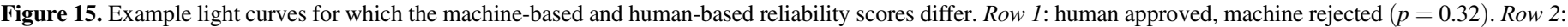

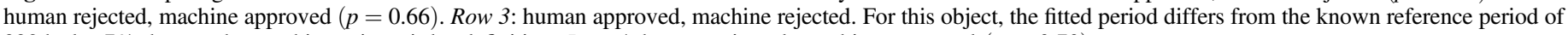
$392 \mathrm{hr}$ by 7\%; hence, the machine rejects it by definition. Row 4: human rejected, machine approved $(p=0.70)$.

period), and (4) the rotational-phase versus phase-angle plot. A single screener is presented with these plots through a plainformatted webpage, allowing for efficient scrolling through the light curves and rapid recording of either a "reliable" or "unreliable" rating for each fitted period. In addition, all light curves in the known-period sample were reinserted into the screening list, with their reference periods removed. These were thus blindly assessed by the screener, independent of their formal (3\% accuracy) classification status.

The black lines in Figure 14 plot the results of the manual screening, in which a total of 10,059 light curves (19\% of the total set) were deemed "reliable." With respect to the machineapproved sample, the human-rated sample is in all cases between roughly a factor of $\sim 1$ and 2 smaller in each bin relative to the features examined in Figure 14. In general, the shapes of the machine-approved and human-approved distributions match fairly closely. Figure 15 shows examples of light curves for which the machine- and human-based classifiers differed in their rating (we focus on very short and very long fitted periods in Figure 15, but many examples exist for intermediate periods as well).

\subsection{Asteroids with Multiple Fitted Periods}

A total of 654 unique asteroids have more than one PTF light curve whose fitted period was labeled as reliable by the vetting process described in the previous sections. These 654 asteroids collectively have 1413 fits (so that the average multiplicity is

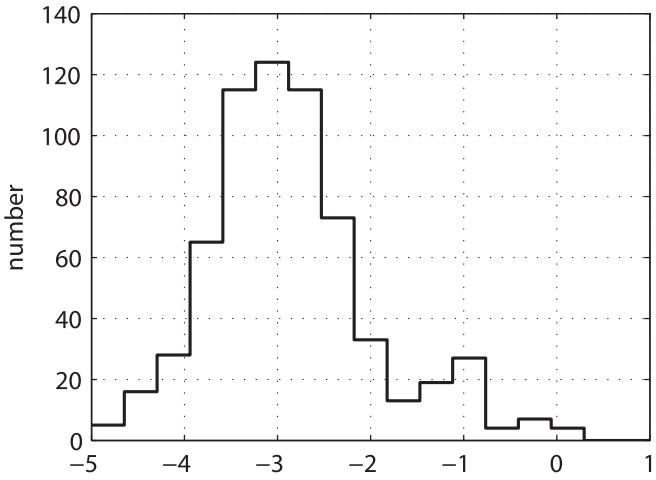

$\log 10$ (multiple frequency fit relative error)

Figure 16. For the 654 unique asteroids having more than one reliable lightcurve fit (either multiple oppositions and/or both $R$ and $g$-band data) we plot the $\log$ of the relative frequency error, defined as the range of the asteroid's fitted periods divided by the geometric mean of its fitted periods. Comparison with Figure 5 suggests that we can deem all cases with error $\lesssim 3 \%$ as consistently recovered periods, and those with greater than $3 \%$ error as inconsistent fits.

2.2 fits per asteroid) and include objects either observed in multiple oppositions and/or in both filters during one or more oppositions. Figure 16 plots the distribution of the relative error in the fitted frequencies of all such multiply fit asteroids, this error being defined as the range of the asteroid's fitted frequencies divided by the geometric mean of its fitted 
frequencies. Just as in Figure 5 (when we compared to literature-referenced frequencies), we see a prominent mode in the histogram peaking at $\sim 0.1 \%$ relative error, with some excess for errors greater than $\sim 3 \%$ error. There are 63 asteroids in particular with relative errors greater than $3 \%$; of these, only four asteroids have more than two fits. If we assume that, in the remaining 59 pairs of disagreeing periods, one of the periods is correct, then the contamination fraction of light curves based on the sample of multiply fit asteroids is $\sim 30 / 1413=4 \%$.

\section{PRELIMINARY LIGHT-CURVE-BASED DEMOGRAPHICS}

In this section we perform a preliminary analysis and interpretation of the demographic trends evident in this work's fitted light-curve parameters. Forthcoming works and papers will more closely examine the population distributions of both rotation and phase-function parameters.

Throughout this section we repeatedly examine variation of light-curve-derived parameters as functions of color index and infrared-derived diameters. In the Appendix we describe the aggregation and characteristics of these two custom data sets (compiled from external sources). The color index quantifies an asteroid's probability of membership in the C-type $(p=0)$ versus S-type $(p=1)$ color-based clusters. Objects that in fact belong to neither $\mathrm{C}$ nor $\mathrm{S}$ groups (e.g., $\mathrm{V}$ types, $\mathrm{D}$ types) will have color indices near $p=0.5$ provided that they are in fact separated from both the C-type and S-type clusters in the 2D color spaces considered (see Appendix).

There are many interesting demographic questions addressable with these light-curve data that-in the interest of space -we do not treat in this work. For example, one could examine relationships between light-curve parameters and orbital elements and/or family membership, proximity to resonances, and so on. We are making all of these light-curve data available electronically (Tables 4 and 5; see Appendix A.3) so that the community may use these data to help explore such science questions.

\subsection{Disclaimer Regarding De-biasing}

The preliminary demographic analyses that follow do not take into account fully de-biased distributions of, e.g., spin rates, amplitudes, or phase-function parameters. The TPRs and FPRs given in the bottom row of plots in Figures 7 and 8 (also, the blue and violet lines in Figure 14) constitute some of the necessary ingredients for producing a fully de-biased data set; however, in this work we do not attempt to compute the debiased distributions.

\subsection{Rotation Rates and Amplitudes}

In Figure 17 we reproduce several of the plots appearing in Pravec et al. (2002 and references therein), using this work's much larger data set (characterized by at least an order of magnitude larger sample of small objects). Both spin rate and amplitude are examined for the 4040 objects having diameter data from infrared surveys. Unlike Pravec et al. (2002), we are not able to individually plot each light curve's data (the $\sim 4000$ points would make the plot difficult to render, as well as difficult to read); hence, we plot these (and other relationships later in this section) using two-dimensional histograms where the intensity of each pixel corresponds to the number of objects in that bin (darker means more, with linear scaling).
Additionally, 2D histograms for which the diameter is plotted on the horizontal axis have their pixel values column-normalized, i.e., all pixels in each column of the histogram sum to the same value. This facilitates the visual interpretation of period and amplitude variation with diameter, as the left-hand side (small-diameter end) of the plots would otherwise saturate the plot.

Following Pravec et al. (2002), we include the geometric mean rotation frequency as computed from a running bin centered on each object. The half-width of the bin centered on each object is either 250 (data points) or the object's distance from the top or bottom of the sorted diameter list, whichever is smallest. This ensures that the geometric mean is not contaminated at the edges of the plot by the interior values, though it also means that more noise exists in these edge statistics. The geometric mean is the more intuitive statistic for the rotation period as compared to the arithmetic mean, since the rotation periods tend to span several orders of magnitude. In addition to the geometric mean, we plot the 16th and 84th percentile values from each running bin.

The basic observed trend regarding rotation rate is that smaller-diameter asteroids rotate faster on average. A slight increase in the rotation rate also appears for objects larger than $\sim 80 \mathrm{~km}$. Binning the data into a coarser set of three diameter bins and normalizing each object's spin rate by the local geometric-mean rate, we see a progression from a nearMaxwellian distribution to a progressively non-Maxwellian distribution for smaller objects. The rotation rates of a collisionally equilibrated population of rotating particles are known to approach that of a Maxwellian distribution (e.g., Salo 1987), which for a population of $N$ objects as a function of rotation frequency $f$ is

$$
n\left(N, f, f_{\text {peak }}\right)=\frac{4 N f^{2}}{\sqrt{\pi} f_{\text {peak }}^{3}} \exp \left(-\frac{f^{2}}{f_{\text {peak }}^{2}}\right),
$$

where $n\left(N, f, f_{\text {peak }}\right) d f$ is the number of objects in the interval $(f, f+d f)$ and $f_{\text {peak }}$ is the peak frequency (i.e., the frequency corresponding to the distribution's maximum).

One way of testing how well a Maxwellian actually fits the data is the two-sided Kolmogorov-Smirnov test (Massey 1951). This test compares an empirical distribution to a reference distribution (e.g., Gaussian, Maxwellian, or another empirical sample) via a bootstrap method. In particular, it computes a statistic quantifying the extent to which the cumulative distribution function differs in the two distributions being compared. In our case, we use Equation (32) to simulate a large sample $\left(10^{5}\right)$ randomly drawn from an ideal Maxwellian distribution and compare this simulated sample against the 99-asteroid sample (of $D>40 \mathrm{~km}$ ) frequencies. Interestingly, this test indicates that our 99 large-asteroid normalized frequencies differ from a Maxwellian at nearly the $10 \sigma$ confidence level, though this could be due in part to the lack of a proper de-biasing of the distribution (see Section 6.1)

All of these trends-including the qualitative resemblance of a Maxwellian but its formal disagreement-were noted previously by Pravec et al. (2002). At the time their $D<10$ $\mathrm{km}$ size bin contained data on only 231 objects, as opposed to our sample of 2,844 asteroids with $D<10 \mathrm{~km}$. Conversely, our $D>40 \mathrm{~km}$ bin contains only 99 objects as compared to the $\sim 400$ large asteroids they took into consideration in comparing to a Maxwellian. 



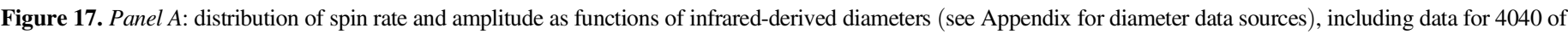

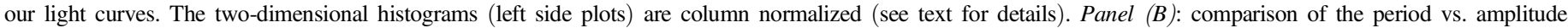
distribution (regular 2D histogram, not column normalized) with max-spin-rate vs. amplitude for a uniform density ellipsoid held together solely by self-gravity.

Steinberg \& Sari (2015) recently described how collisional evolution of large asteroids should actually lead to a Lévy distribution, which has a significantly longer tail than a Maxwellian distribution having the same peak. They compared their theory to spin rates of $D \geqslant 10 \mathrm{~km}$ asteroids from the LCDB and found in general that the Lévy distribution fails to fit the spin distribution of large asteroids, suggesting that there may be a significant primordial component to the spin distribution. Potential primordial contributions to the angular momentum of asteroids were explored by Harris \& Burns (1979) and later authors.

Our amplitude distribution contains an obvious observational bias (see Section 6.1) in that amplitudes less than $\sim 0.1-0.2$ mag are generally ill fit by our modeling procedure (see Figure 7) and thus significantly underrepresented in our sample of reliable light curves considered here. Nonetheless, we see a clear trend of smaller asteroids exhibiting larger rotational amplitudes, consistent with the idea that larger bodies have sufficient surface gravity to redistribute any loose mass to a more spherical shape.

As we have done for the normalized frequency distribution, we plot diameter-binned normalized amplitudes against a Maxwellian distribution, this time merely to guide the eye as opposed to validating any hypothetical physical interpretation. The fact that the normalized amplitude distributions do not deviate too drastically from the Maxwellian shape at smaller diameters indicates that the spread in the amplitude distribution is proportional to its mean value, a basic property of the Maxwellian distribution, hence the good agreement. Carbognani (2010) provides a recent analysis of asteroid rotation amplitudes and highlighted a similar increase in both the amplitude's mean and spread with decreasing diameter.

Panel (B) of Figure 17 shows the distribution in periodversus-amplitude space, in which we can plot all 9033 light curves, including those lacking a diameter estimate. Contours representing the maximal spin rate of a body held together solely by self-gravity of certain uniform densities are overplotted. Our data as a whole do not appear to populate the region beyond the $\sim 2 \mathrm{~g} \mathrm{~cm}^{-3}$ contour. Later in this section we will re-examine this behavior separately for the two major taxonomic classes.

\subsection{Phase Functions and Bond Albedos}

We consider any of the 54,296 fitted PTF light curves to have a reliably fit phase function if both of the following conditions are satisfied: 
1. The light curve is one of the 9033 having a reliable period fit, or its fitted amplitude (for the $G_{12}$ model) is less than $0.1 \mathrm{mag}$ (the latter is true for 1939 light curves, only 39 of which have reliable periods).

2. The light curve is fit using data from at least five phaseangle bins of width $\Delta \alpha=3^{\circ}$. These five bins need not be contiguous, and they need not include phase angles in the region where opposition surges are typically measured (i.e., $\alpha \lesssim 10^{\circ}$ ).

The above two criteria are met by 3902 out of the 54,296 PTF light curves. Of these, 1648 have an infrared-based diameter available, 651 have a color index available, and 361 have both a diameter and color index.

Figure 18 details the distributions of the fitted phase parameters $G_{12}, G, \beta$, and $C$ against the color index, bond albedo, and in 1D histograms with color-based taxonomic subsets. Though the phase parameters are all correlated with color index and with bond albedo, none of the 1D phaseparameter distributions (right column of plots) exhibit bimodality alone, whereas the bond albedo (bottom right plot) does show significant bimodality. The red and blue histograms consist of all asteroids having color metric either less than 0.25 (C types) or greater than 0.75 (S types). The $G$ and $(\beta, C)$ phase parameters are only plotted for those light curves that also have a $G_{12}$ solution. Not every light curve produced a solution for all three of the phase-function models; hence, the sample sizes for the $G$ and $(\beta, C)$ models include a slightly reduced number of data points.

We reiterate our statement from Section 3.2.1 that the bond albedo $A_{\text {bond }}$ is a more fundamental (i.e., intensive rather than extensive) property than is the geometric albedo $p_{V}$, hence our focus on $A_{\text {bond }}$ here. The bond albedo is computed using Equation (8) together with Equation (15) and makes use of our PTF-derived absolute magnitudes $-H$ from the $G_{12}$ fit in particular-as well as the phase integral $q$ of Equation (8), also computed directly from the $G_{12}$ fit for $\phi$. In particular,

$$
q\left(G_{12}\right)= \begin{cases}0.2707-0.236 G_{12} & \text { if } G_{12}<0.2 \\ 0.2344-0.054 G_{12} & \text { otherwise }\end{cases}
$$

\subsubsection{Taxonomy from Light-curve Data}

We use the distribution of bond albedo versus $G_{12}$ to define another taxonomic metric analogous to the color index. In particular, we apply the same clustering analysis to this distribution as we did for the seven 2D color distributions in the Appendix. This procedure assigns to every object in the $A_{\text {bond }}$ -versus- $G_{12}$ diagram a probability of membership in each of two clusters (color-coded blue and orange in Figure 19). The cluster centers are fit by the algorithm, and the output class probability of a given data point relates to its distance from these cluster centers. Probabilities near 0 represent likely C-type class membership, while probabilities near 1 represent likely S-type membership. We refer to this new metric as the photometric index; it complements the color index as another proxy for taxonomy. There are 361 asteroids with both a photometric index and color index available (Figure 19, right plot $)$; the two indices are clearly correlated $\left(\rho_{\text {Spearman }}=0.73\right.$, $>10 \sigma$ significance). Note that asteroids only have a defined photometric index if they have an infrared-derived diameter available, so that $A_{\text {bond }}$ is defined.

\subsubsection{Wavelength Dependence}

Observational evidence for the reddening of asteroid colors with increasing phase angle is discussed by Sanchez et al. (2012 and references therein). Color variation with phase angle can be equivalently stated as variation of the phase function with wavelength. Asteroids that have PTF light curves in both of the survey's filters ( $R$ and $g$ band) allow us to investigate this phenomenon. We note, however, that Sanchez et al. (2012) describe phase reddening as being more pronounced at longer wavelengths $(>0.9 \mu \mathrm{m})$ and larger phase angles $\left(\alpha>30^{\circ}\right)$, such that a priori we should not expect a very pronounced effect (if any) in the visible-band PTF data.

Similar to the complication associated with comparing spin amplitudes from multiple oppositions (Section 3.1.1), an asteroid's mean color can potentially change if the spin axis varies with respect to our line of sight from year to year. Hence, we choose not to compare $R$-band and $g$-band phase-function fits from different oppositions. Aside from this constraint, we adopt the same two reliability selection criteria stated in Section 6.3, with a slight modification of requirement $\# 2$ : here we allow four or more phase-angle bins of width $\Delta \alpha=3^{\circ}$, as opposed to the previous sections' five-bin requirement, because of the small sample size.

There are 92 asteroids with both $R$-band and $g$-band phasefunction fits acquired during the same opposition that meet the above criteria. For each asteroid we difference the $R$-band $G_{12}$ value from the $g$-band $G_{12}$ value. The mean of this difference is $-0.004_{-0.14}^{+0.19}$, indicating (for the whole sample) no significant non-zero difference between the two bands' $G_{12}$ values. Likewise, for $\beta$, we compute a difference of $0.002_{-0.003}^{+0.008}$, also consistent with zero difference between the bands.

Since these fits provide absolute magnitudes in each band (i.e., $H_{g}$ and $H_{R}$ ), we compute the color $H_{g}-H_{R}$ for the 92asteroid sample. Figure 20 shows that the distribution of this color is bimodal, suggesting that it is a viable proxy for taxonomy. This is further supported by the strong correlation between $H_{g}-H_{R}$ and the $R$-band $G_{12}$ value. No correlation is seen, however, between $H_{g}-H_{R}$ and the difference between the two bands' $G_{12}$ value or $\beta$ values.

\subsection{Spins and Amplitudes versus Taxonomy}

The union of the color-index data (see Appendix) and photometric-index data (Section 6.3.1) provides significantly better taxonomic coverage of the PTF light curves (Figure 21). With this composite taxonomic information in hand, we can repeat the spin-amplitude analyses of Section 6.2 (Figure 17), this time considering the C-type and S-type groups separately. We define objects with one or both of the indices less than 0.25 as $\mathrm{C}$ type and greater than 0.75 as $\mathrm{S}$ type. We detail the resulting 1795-object taxonomically classified sample in Figure 22. There were 20 asteroids with conflicting colorbased and photometric-based classifications that are not included in this 1795-object sample.

The one-dimensional histogram in Figure 22 indicates that S-type asteroids dominates the smallest objects with data in PTF while $C$ type dominates the largest. This reflects the fact that the survey's upper and lower sensitivity limits are defined in terms of absolute magnitude $H$ (affected by albedo) rather than physical diameter, i.e., S-type asteroids larger than $\sim 50 \mathrm{~km}$ will tend to saturate the PTF detector, while C-type asteroids fainter than $\sim 5 \mathrm{~km}$ will usually fall below the 

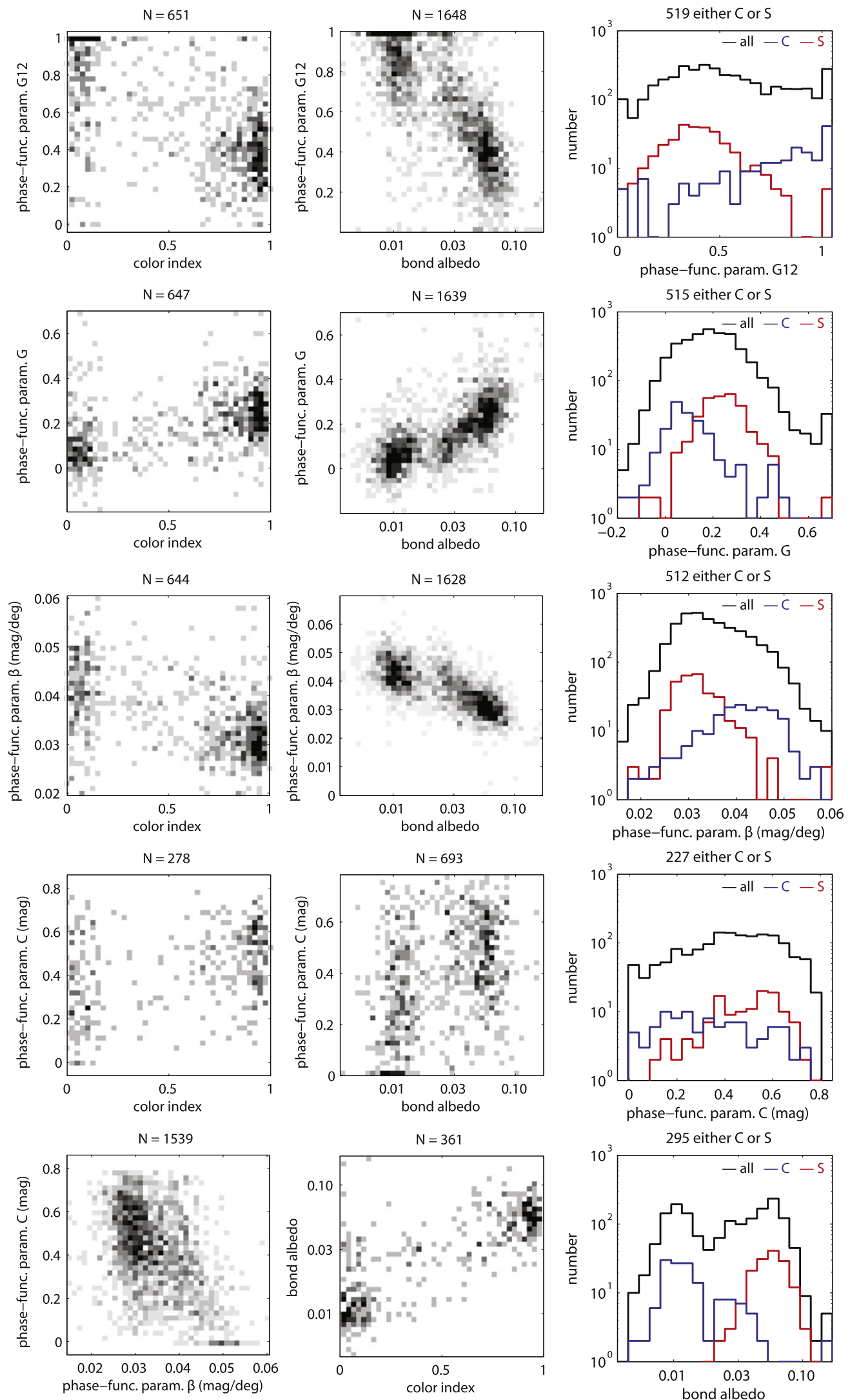

Figure 18. Various fitted phase-function parameters plotted against color index and bold albedo (two-dimensional histograms; the total number of light curves in each plot is stated above it as $N=\ldots$. . In the right column of plots, one-dimensional distributions with the color-index classified objects plotted separately. In the right column of $1 \mathrm{D}$ histograms, $\mathrm{C}$ and $\mathrm{S}$ types are defined as objects with color indices less than 0.25 and greater than 0.75 , respectively. 

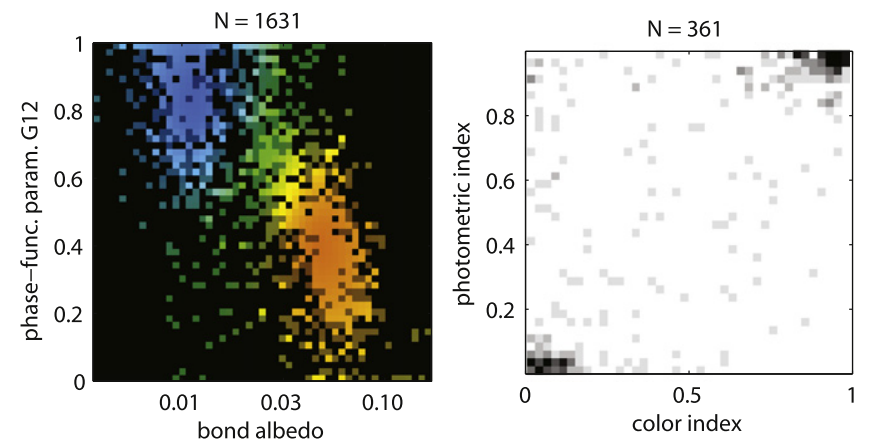

Figure 19. Left: we perform the same clustering analysis used in defining the color index (see appendix), this time on the $G_{12}$ vs. $A_{\text {bond }}$ distribution, which contains 1631 PTF light curves, all of which have IR-derived diameters and reliable phase functions. The output of this clustering analysis is the photometric index, which, analogous to the color index, is a number between 0 (C type) and 1 (S type) quantifying to the class membership of each constituent asteroid data point. Right: correlation between the color index and our photometric index, a comparison that can be made for 361 objects. Note that most data are in the lower left and upper right corners.
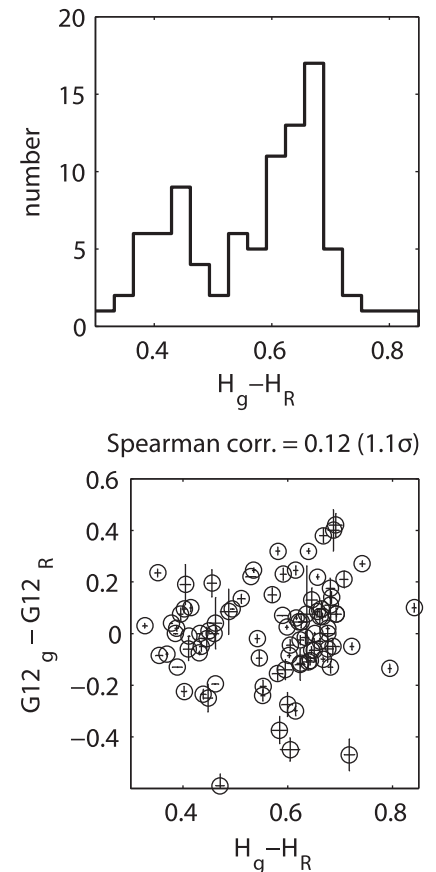



Spearman corr. $=0.00(0.05 \sigma)$

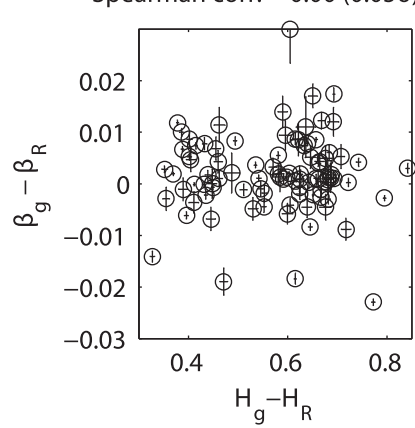

Figure 20. For the 92 asteroids with both $R$-band and $g$-band light-curve fits from the same opposition, we use the resulting difference in the absolute magnitudes $H_{g}-H_{R}$ as a proxy for taxonomy. This color distribution is qualitatively bimodal (top left), and the correlation with $G_{12}$ is very robust (top right). We detect no significant difference in the $G_{12}$ and/or $\beta$ parameters between the two bands, both in the sample as a whole and as a function of the $H_{g}-H_{R}$ color.

detection limit. Adding to this effect is the fact that $\mathrm{S}$ types mostly occupy the inner main belt, where they are brighter by virtue of smaller heliocentric and geocentric distances, as compared to the usually more distant $C$ types. While the two classes have similar representation in the sample (882 S types versus $913 \mathrm{C}$ types), their true population ratio also affects the relative numbers.

The right-hand side plots in Figure 22 show rotation rate and amplitude versus diameter separately for the two taxonomic groups. Rather than plot a two-dimensional histogram as was
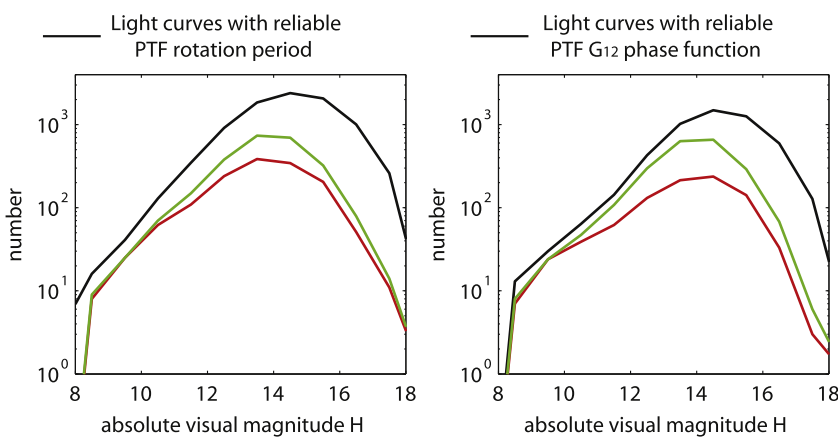

- Has color index



Figure 21. Added completeness from supplementing the color index with the photometric index among asteroids having PTF light curves. Both indices are a proxy for the taxonomic type. The left- and right-hand plots apply separately to the subset labeled by the black line above each column.

done in Figure 17, for readability we here just plot the geometric mean and percentiles, computed by exactly the same running-bin method described in Section 6.2. The most prominent trend is that among $5 \lesssim(D / \mathrm{km}) \lesssim 20$ asteroids, C types appear to rotate slower than $\mathrm{S}$ types and have larger amplitudes than $\mathrm{S}$ types. Assuming that both asteroid groups share the same mean angular momentum, the discrepancy could reflect the $\mathrm{C}$ types' ability to more efficiently redistribute material away from their spin axis, thereby increasing their moment of inertia (amplitude) while decreasing their angular rotation rate (i.e., a simple manifestation of conservation of angular momentum).

The above-stated assumption of a common mean angular momentum between $\mathrm{C}$ and $\mathrm{S}$ types is merely a simple case and is neither unique nor rigorously motivated. More careful consideration of, e.g., plausible ranges of internal tensile strengths of the two types could easily lead to more diverse scenarios wherein the two groups actually have different angular momenta and the observed spin-amplitude trends. As noted earlier (Section 6.2), large asteroids in general appear to have retained a significant primordial component in their spin distribution (Steinberg \& Sari 2015); it is therefore important that differences in the origin of $\mathrm{C}$ types and $\mathrm{S}$ types (accretionary, temporal and/or spatial) be taken into account along with differences in collisional evolution and differing contributions from radiative forces like YORP. Simulations of the main belt's origin, such as the Grand Tack family of models (Walsh et al. 2011), should ultimately be modified to track particle spin evolution as well as orbits.

We also reproduce the period-versus-amplitude plot first shown in Figure 17, this time plotting separately the two taxonomic groups. The $\mathrm{S}$ types show a clearer cutoff at the $2 \mathrm{~g} \mathrm{~cm}^{-3}$ contour line, suggesting that they may in general be of 

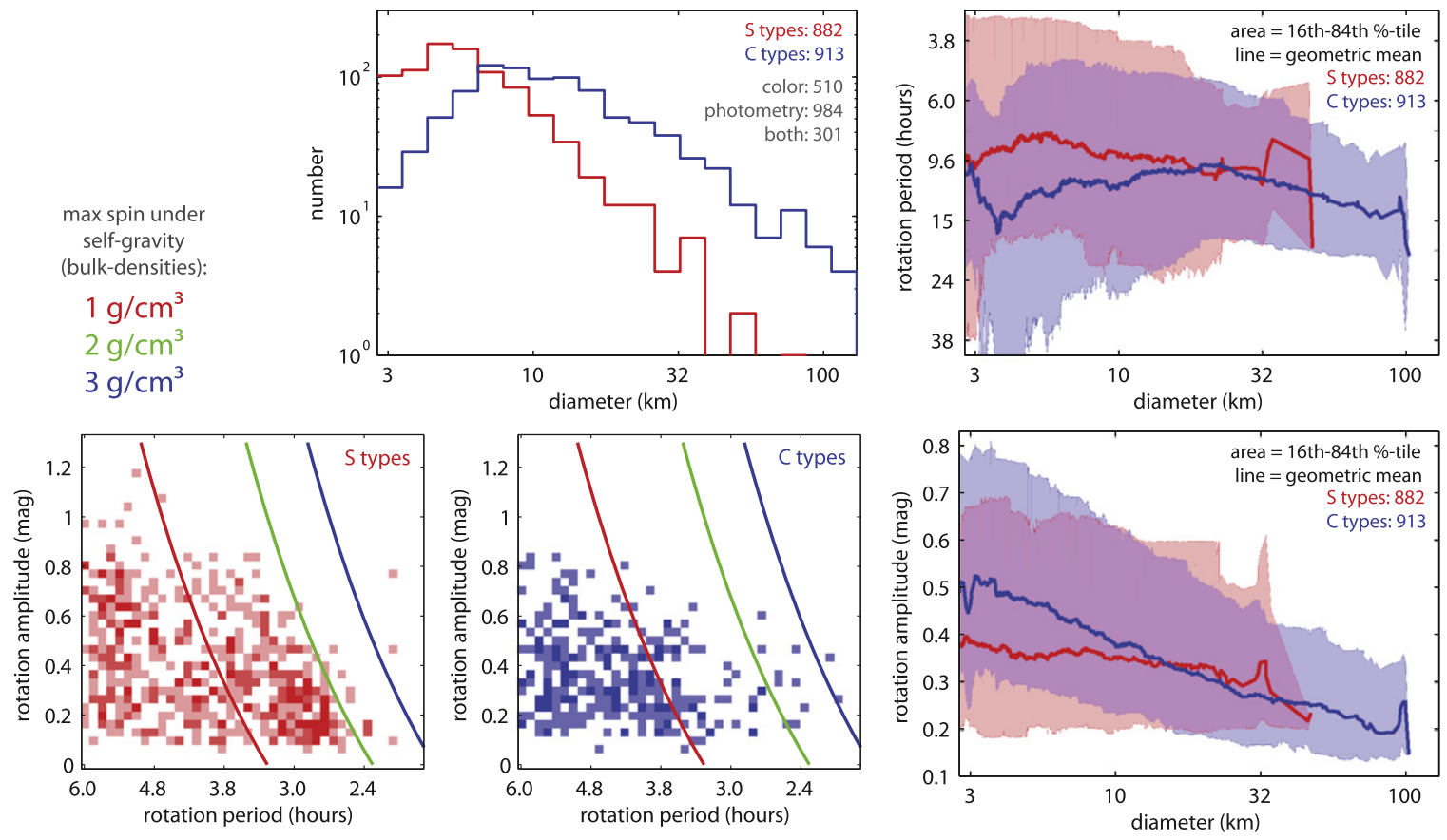

Figure 22. Taxonomic dependence on spin rate and amplitude, also vs. diameter, using the union of the color-index- and photometric-index-based C/S taxonomy.

greater bulk density than the $\mathrm{C}$ types, which show a softer boundary in this period-versus-amplitude space, the precise location of which appears to be somewhere between 1 and $2 \mathrm{~g} \mathrm{~cm}^{-3}$. Note that comparison to these density contours is only valid if the asteroids in consideration are held together mostly by self-gravity and approximated as fluids (as opposed to having significant internal cohesive or frictional resistance). These results are in general agreement with existing asteroid density estimates (Carry 2012 and references therein). Results from an independent analysis of a smaller, more densely sampled set of PTF asteroid light curves (Chang et al. in review; a study that follows closely the approach of Chang et al. 2014a) agree with the $\mathrm{C}$ type versus $\mathrm{S}$ type rotation rate discrepancy discussed here.

\section{COMPARISON TO MPC-GENERATED MAGNITUDES}

Absolute magnitudes available through the Minor Planet Center (MPC) and JPL Solar System Dynamics ${ }^{21}$ websites are fit using all available survey/observer-contributed photometry. These $H$ magnitudes are used in various online ephemeris tools to compute predicted $V$ magnitudes to accompany astrometric predictions. Their model assumes no rotational modulation, uses the Lumme-Bowell $G$-model (Section 3.2.2), and-with the exception of $\sim 100$ large objects (nearly all with $D>30$ $\mathrm{km}$ )-assumes a constant $G=0.15$ for all asteroids. Our results (Figure 18 second row of plots) show that the $G=0.15$ approximation does indeed agree well with the peak of the distribution of fitted $G$ values. The PTF-fitted $G$ values obviously, however, show some spread and variation with taxonomy. In this section we explore the resulting differences in the absolute magnitudes $H$ and in predicted magnitudes.

\footnotetext{
${ }^{21}$ http://ssd.jpl.nasa.gov
}

\subsection{Filter Transformations}

In order to compare the MPC-listed $\left(H_{\mathrm{MPC}}\right)$ magnitudes, which are in $V$ band, with PTF's absolute magnitudes $\left(H_{\mathrm{PTF}}\right.$, corresponding to the $G$-model fit), which are in either $R$ or $g$ band, we must first compute an approximate transformation from $V$ band to each PTF band. While some transformations are given by Ofek et al. (2012a), we here prefer to empirically estimate these using actual asteroid photometry from both PTF and the MPC, rather than generating them from the more general transformations of Ofek et al. (2012a).

Figure 23 plots $H_{\mathrm{PTF}}-H_{\mathrm{MPC}}$ for asteroids whose PTFderived $G_{\mathrm{PTF}}$ is in the range $0.1<G_{\mathrm{PTF}}<0.2$. By restricting the comparison to objects with fitted $G_{\mathrm{PTF}}$ values close to 0.15 , we in principle select $H_{\mathrm{MPC}}$ magnitudes for which the MPC's $G_{\mathrm{MPC}}=0.15$ assumption is actually valid (none of the asteroids in Figure 23 have MPC-listed $G$ values other than the default 0.15 ). Furthermore, we only consider (in Figure 23) asteroids with PTF data in at least three phase-angle bins of $\Delta \alpha=3^{\circ}$ and either a reliable period or fitted amplitude less than $0.1 \mathrm{mag}$.

Comparing the $H_{\mathrm{MPC}}$ and $H_{\mathrm{PTF}}$ magnitudes for this specific subset of asteroids, we obtain approximate transformations $R=V+(0.00 \pm 0.10)$ and $g=V+(0.55 \pm 0.16)$. The $1 \sigma$ uncertainties of 0.10 and $0.16 \mathrm{mag}$ plausibly include a combination of the photometric calibration uncertainties of the MPC data (coming from a variety of surveys/observers), variation in $H$ magnitude of a given asteroid between different oppositions (the MPC fits combine data possibly acquired at different viewing geometries), and the range of $G_{\mathrm{PTF}}$ used in selecting the asteroids in this sample. Consideration of a range of $G_{\mathrm{PTF}}$ values is equivalent to considering a range of asteroid colors (see the color-versus- $G$ correlation seen in Figure 18). Hence, the uncertainties in these transformations also encompass the variation that might otherwise be formally fit in a color term for the transformations. Such a color term for $R$ to $V$ would almost certainly be less significant than that of $g$ to $V$, as the 

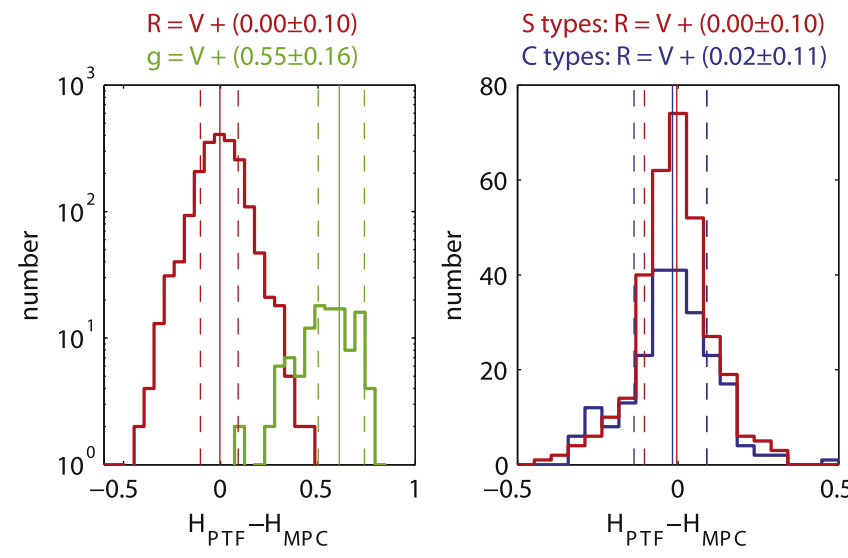

Figure 23. Left: transformations between MPC $V$ band and the PTF $R$ and $g$ bands for asteroids, based on the difference between MPC-fitted and PTF-fitted $H$ magnitudes for asteroids whose PTF-fitted $G$ values are in the range $0.10<G<0.20$, as well as other PTF-coverage constraints (see text). Right: $R$-band data only, with $\mathrm{S}$ and $\mathrm{C}$ types defined with color and/or photometric indices (again using the $<0.25$ and $>0.75$ index thresholds).

former transformation is already zero within uncertainties. The larger uncertainty in the $g$ to $V$ transformation is likely attributable to both the smaller sample size and the fact that the $V$ bandcenter is further displaced from $g$ than from $R$, such that color variation has a more pronounced effect.

Given the above-computed transformations, and the fact that $89 \%$ of our fitted light curves are in $R$ band, we proceed using only $R$-band light-curve fits, which we compare directly against MPC magnitudes (or formally, after applying the transformation of zero). A detail of the color dependence of the $R$ to $V$ transformation appears in the right plot of Figure 23; the mean transformation differs slightly between $\mathrm{S}$ and $\mathrm{C}$ types but not at a level comparable to the uncertainty in either.

\subsection{Absolute Magnitudes}

In Figure 24 we show the relative error in the MPC absolute magnitudes as compared to the PTF magnitudes, for all 1630 light curves with sufficient phase-angle coverage in PTF (with the five-bin phase-angle criterion). These errors should reflect not only any discrepancy due to the different phase function models (PTF's $G_{12}$ versus MPC's $G$ ) but also variation in absolute photometric calibrations (within the MPC data internally and/or between the MPC and PTF data sets). The $0.1 \mathrm{mag}$ uncertainty in the $R$ to $V$ band transformation has a prominent contribution to the errors shown here (the mean and 84th percentile of the errors expected from the $0.1 \mathrm{mag}$ transformation uncertainty alone are shown as yellow dashed lines and assume $\left.p_{V}=0.07\right)$. The green line (computed mean) and upper red line (84th percentile) indicate that the errors are $\sim 1 \%$ greater than those expected from the transformation uncertainty alone, though this increases slightly for the largest $(D>30 \mathrm{~km})$ objects. Note that many of these largest asteroids are more frequently observed by programs other than the major sky surveys; these smaller facilities tend to use smaller aperture telescopes and different absolute calibration standards, which would contribute to the error.

\subsection{Predicted Apparent Magnitudes}

Instead of comparing just the fitted $H$ magnitudes, for every light curve with a reliable PTF-fitted phase function we also
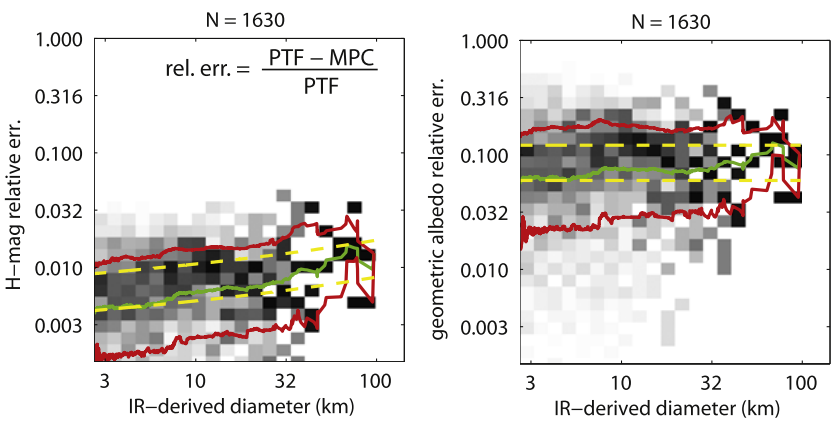

Figure 24. Errors in the MPC-listed absolute magnitudes relative to the PTF $H$ values (in $R$ band and using the $G_{12}$ fit's $H$ value), only considering asteroids with IR-derived diameters. On the right is the corresponding geometric albedo relative error. Pixels in the $2 \mathrm{D}$ histograms shown here are column normalized. The running-bin geometric mean and 16th and 84th percentiles are shown as green and red lines. Yellow dashed lines are the mean and 84th percentile expected from the $0.1 \mathrm{mag}$ transformation uncertainty alone (for $7 \%$ geometric albedo).



Figure 25. Comparison of the rms residuals, with respect to the PTF $\left(H, G_{12}\right)$ plus rotation fit and the MPC $(H, G)$ fit, for all light curves having a reliable $R$ band PTF phase-function fit.

compare the rms residual of all PTF data in that light curve with respect to both our $G_{12}$-fit-predicted $R$ magnitude and the MPC $(G=0.15)$ predicted $V$ magnitude. Our fit includes more fitted parameters and obviously should result in smaller residuals; Figure 25 shows that we see a factor of $\sim 3$ smaller residuals in particular using the PTF fit. Note that if the $0.1 \mathrm{mag} R$-to- $V$ transformation uncertainty were the only significant contributor to the MPC residuals, then their peak would instead be at $\sim 0.07$ mag rather than $\sim 0.25 \mathrm{mag}$. Ignored rotational modulation and inaccurate phase functions move the MPC residuals distribution to higher rms values.

The lower rms residuals afforded by the PTF light-curve model permit a more sensitive search for low-level transient activity (e.g., collisional events, cometary brightening) in these asteroids. For example, Cikota et al. (2014) perform a search for active main-belt asteroids using photometric residuals of all MPC data taken with respect to the MPC-predicted apparent $V$ magnitudes. We currently are pursuing a similar analysis using these PTF light curves, as a follow-up to the morphology-based search already completed with PTF (Waszczak et al. 2013). A hybrid approach, wherein morphological measurements are made on stacked images of asteroids that have reliable lightcurve fits, could further reveal this kind of subtle activity.

\section{SUMMARY}

From 5 yr of PTF survey data we have extracted over 4 million serendipitous detections of asteroids with known orbits. We fit a photometric model to $~ 54,000$ light curves, each consisting of at least 20 observations acquired within a given opposition in a single filter. We adopt a second-order (four-term) 
Fourier series for the rotation component and fit three distinct phase-function models. We assess the reliability of our retrieved rotation periods by subjecting them to both an automated classifier and manual review. Both vetting processes are trained on a sample of $\sim 800$ asteroids with previously measured spin periods that also occur in our sample. We consider the intersection of the two screened samples for subsequent analysis.

Preliminary analysis (on distributions that are not de-biased) of the rotation period versus diameter confirms the previous finding that asteroids smaller than $\sim 40 \mathrm{~km}$ do not conform to a Maxwellian distribution in their normalized spin frequencies. Phase-function parameters are shown to correlate strongly with the bond albedo. None of the phase function parameters display bimodality in their measured distributions, however. Together with the bond albedo, we use the phase-function data to define a new taxonomic metric based solely on single-band lightcurve properties together with infrared-derived diameters $\left(G_{12}\right.$ and $\left.A_{\text {bond }}\right)$. This metric complements the color-based index established previously by many visible-color and spectroscopic surveys. Combining these color- and photometry-based taxonomic indices allows us to separately examine the spin and amplitude distributions of the C-type and S-type asteroids with the largest possible sample sizes. Doing so reveals that, among small objects $(5 \mathrm{~km}<D<20 \mathrm{~km})$, the $\mathrm{C}$ types show larger amplitudes and slower spin rates. If the two populations shared a common angular momentum distribution, this could be interpreted as the two compositional types' differing tendencies to redistribute mass away from their spin axes. Comparison of the spin-amplitude distribution with contours of maximal spin rates for cohesionless bodies suggests that almost all asteroids are less dense than $\sim 2 \mathrm{~g} \mathrm{~cm}^{-3}$, with $\mathrm{C}$ types displaying a potentially less dense upper limit of between $1-2 \mathrm{~g} \mathrm{~cm}^{-3}$.

Finally, our fitted absolute magnitudes differ from those generated by the Minor Planet Center's automated fitting procedures, though the precise discrepancy is difficult to ascertain given uncertainty in the transformation between PTF $R$ band and the MPC's $V$ band. The utility in using our model to predict asteroid apparent magnitudes is seen in the threefold reduction in rms scatter about our model relative to the fiducial $G=0.15$ model that neglects rotation. This reduced scatter is an essential prerequisite for sensitive searches for cometary, collisional, and other transient activity in what would otherwise be regarded as quiescent asteroids-potentially even bright objects.

This work uses data obtained with the $1.2 \mathrm{~m}$ Samuel Oschin Telescope at Palomar Observatory as part of the PTF, a scientific collaboration between the California Institute of Technology (Caltech), Columbia University, Las Cumbres Observatory Global Telescope Network, Lawrence Berkeley National Laboratory, the National Energy Research Scientific Computing Center, the University of Oxford, and the Weizmann Institute of Science (WIS).

Some data in this work (also from the $1.2 \mathrm{~m}$ Oschin Telescope) were obtained as part of the iPTF project, a collaboration among Caltech, the Kavli Institute for the Physics and Mathematics of the universe, Los Alamos National Laboratory, the Oskar Klein Centre, the University System of Taiwan, the University of Wisconson Milwaukee, and WIS.

A. Waszczak has been supported in part by the W. M. Keck Institute for Space Studies (KISS) at Caltech. E.O.O. is incumbent of the Arye Dissentshik career development chair and is grateful to support by grants from the Willner Family Leadership Institute Ilan Gluzman (Secaucus NJ), Israeli Ministry of Science, Israel Science Foundation, Minerva and the I-CORE Program of the Planning and Budgeting Committee, and The Israel Science Foundation.

This work also makes use of data products from the Wide-Field Infrared Survey Explorer, a joint project of the University of California Los Angeles and the Jet Propulsion Laboratory (JPL)/Caltech, funded by NASA. This work also makes use of data from NEOWISE, which is a project of JPL/ Caltech, funded by the Planetary Science Division of NASA.

This work also makes use of data from the SDSS, managed by the Astrophysical Research Consortium for the Participating Institutions and funded by the Alfred P. Sloan Foundation, the Participating Institutions, the National Science Foundation, the US Department of Energy, NASA, the Japanese Monbukagakusho, the Max Planck Society, and the Higher Education Council for England.

Lastly, we thank an anonymous reviewer for helpful comments and feedback.

\section{APPENDIX}

\section{A.1 Multi-survey Visible-band Color Index}

The purpose of this Appendix section is to introduce a onedimensional color metric, based on data from seven different colorimetric asteroid surveys, which quantifies an asteroid's first-order visible-band color-based taxonomy as a number between 0 (C-type endmember) and 1 (S-type endmember). Our primary motivation for doing this is to enable a uniform comparison of PTF light-curve-derived parameters with color spanning from the brightest/largest objects $(H \approx 8-9 \mathrm{mag}$, or $D \approx 125-80 \mathrm{~km}$ diameters) down to PTF's detection limit for main-belt asteroids $(H \approx 16 \mathrm{mag}$, or $D \approx 2-4 \mathrm{~km})$. Figure 26 panel A shows that the fraction of PTF light curves with color information increases by a factor of $\sim 3$ among large asteroids when all seven surveys are considered, whereas for smaller objects the Sloan Digital Sky Survey's (SDSS; York et al. 2000; Ivezić et al. 2002; Parker et al. 2008) moving-object catalog provides essentially all of the color information.

The seven surveys we use are described in Table 3. All of these surveys contain at least two independent color measurements, and when plotting their data in these two-dimensional spaces (or 2D subspaces defined by properly chosen principal components or spectral slope parameters), the first-order C-type and S-type clusters are in all cases prominently seen (Figure 26, panel B). To each such 2D color distribution we apply a two-dimensional fuzzy c-means (FCM) clustering algorithm (Bezdec 1981; Chiu 1994). For each survey data set, FCM iteratively solves for a specified number of cluster centers (in our case, two) in $N$ dimensions (in our case, one dimension) by minimizing an objective function that adaptively weights each datum according to the robustness of its membership in a given cluster. The FCM output includes computed cluster centers and, for each datum, the probability that the datum belongs to each cluster (this being related to the datum's distance from each cluster center).

In the color-distribution plots of Figure 26, panel B (the plots with black backgrounds arranged diagonally), each pixel/bin is colorized according to the average cluster-membership 



SMASS-1 slop


Has SDSS color data


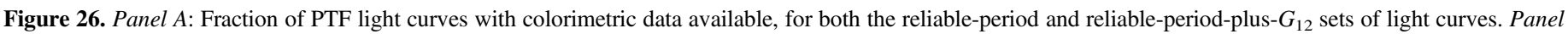
$B$ : Two-dimensional color distributions for seven surveys, and correlations of FCM-clustering-derived classifications between all pairs of surveys.

probability of asteroids in that pixel. Blue indicates high probability of membership in cluster 1 , while orange represents high probability of membership in cluster 2 .

Our color index provides a more quantitative label than that offered by popular letter-based taxonomic systems (e.g., Bus et al. 2002 and references therein). Several such letter-based nomenclatures were in fact defined on the basis of one or more of these seven surveys, oftentimes using a method similar to the clustering technique we use here. We identify our blue cluster with C-type asteroids and our orange cluster with S-type asteroids, though we make this association purely for connection/compatibility with the literature. This is because our computed clusters have their own unique identity/ definition, formally distinct from that given in any other work. Our clusters' definitions are nonetheless completely specified/ reproducible by the FCM algorithm we used to compute them.

In reducing the taxonomic classification to a single number defined by the two most prominent groups ( $\mathrm{C}$ and $\mathrm{S}$ types), we lose the ability to distinguish secondary classes like $\mathrm{V}$ types, D types, and so on. If such a sub-group is separated from both of 
Table 3

Asteroid Colorimetry Data Sets Used in Computing This Work's C/S Color Metric

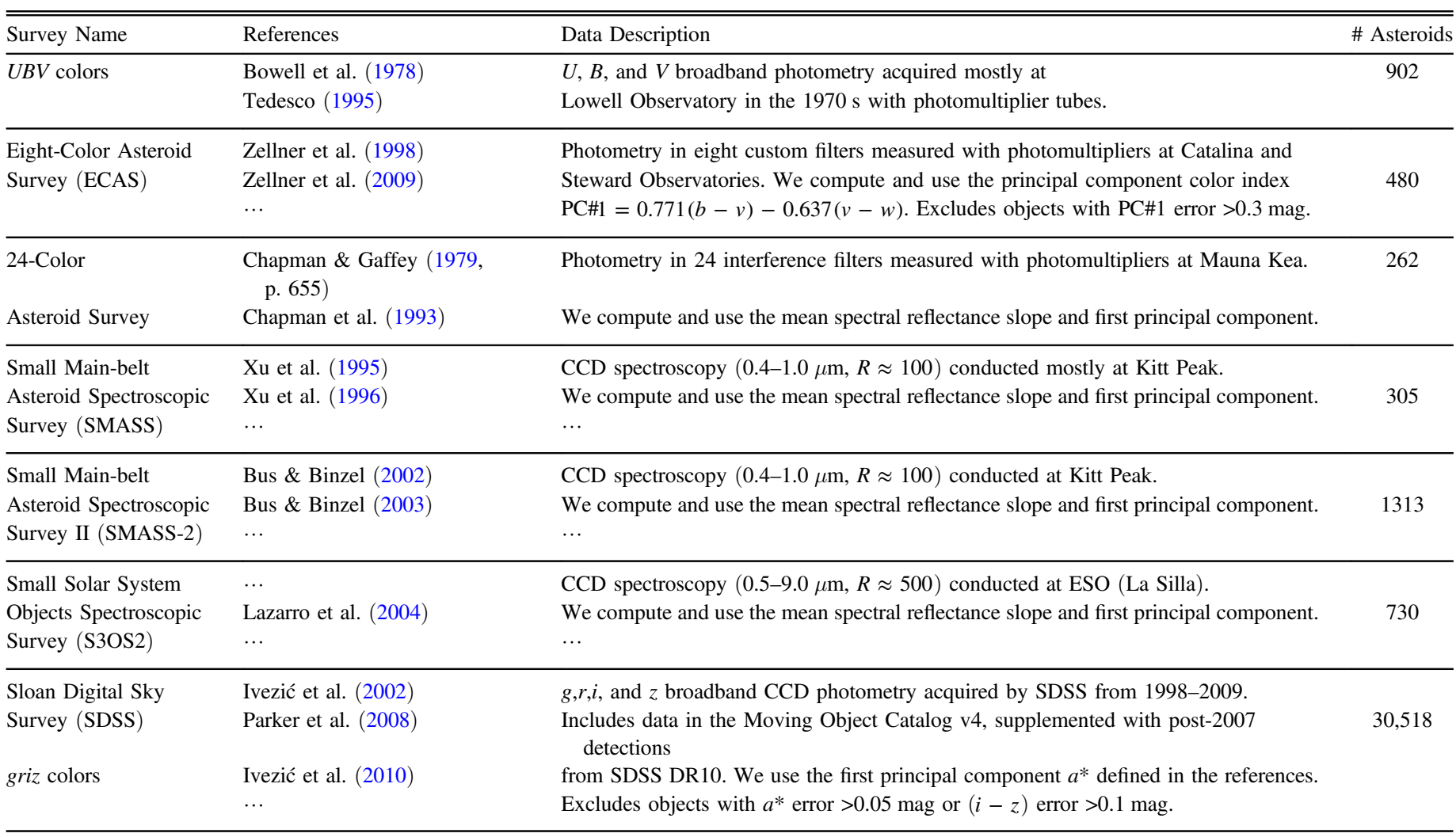

Note. These data sets are visualized in Figure 26.

the two main clusters, its members will be assigned membership scores of close to 0.5. For example, in the SDSS $a^{*}$ versus $i-z$ complex, the clearly seen V-type "tail" protruding down from the S-type cluster appears mostly green in color, reflecting its intermediate classification. Likewise for the less clearly seen $\mathrm{D}$ types, which in the SDSS plot lie above the S types and to the right of the $\mathrm{C}$ types (again in a green-colored region). The orders of magnitude lower numbers of such secondary types make them mostly irrelevant for the purpose of this analysis.

We compute the numerical uncertainty (variance) of a given asteroid's cluster-membership score in a particular survey by performing many bootstrapped trials wherein we first randomly perturb all data points by random numbers drawn from Gaussian distributions whose width are the quoted $1 \sigma$ measurement (i.e., photometric) uncertainties in each of the two dimensions, and then repeat the FCM analysis on the perturbed data. The variance in each object's reported cluster probability is then computed after a large number of bootstrap trials.

Some asteroids appear in only one of the seven surveys; for such objects the color index is simply its cluster-membership score in that particular survey. For asteroids appearing in multiple surveys, we take the variance-weighted average of the multiple membership scores (and compute that composite score's variance by summing the component variances in inverse quadrature, as usual).

The many off-diagonal plots in Figure 26, panel B compare the cluster-membership scores of all asteroids appearing in all possible survey intersections. The number of asteroids in each survey (and in the intersection of each survey pair) appears above each plot $(N=\ldots)$. The survey-pair distributions are 2D histograms where higher densities of data points correspond to black pixels/bins and low density or lack of data points is white. Evidently all possible survey combinations contain at least some asteroids (several share hundreds), and in all cases the individual taxonomic indices (on the horizontal and vertical axes) correlate strongly, confirming the consistency of the cluster membership between surveys.

In Figure 27 we illustrate some useful applications of this color index by comparing it with various asteroid surface observations. One of these quantities (SDSS $a^{*}$ color) was used in computing the color index, so its correlation with the clustering index is expected and thus confirmed.

In the leftmost plot of Figure 27, asteroid photometry from


in preparation), is normalized by the nominal $G=0.15$ phasemodel (Section 3.2.2) predicted brightness at the time of the GALEX observations, and the resulting NUV- $V$ color evidently correlates with the visible color index. This indicates that asteroid reflectance slopes in the visible persist into the UV.

Figure 27 also plots our color index against the W1-band geometric albedo derived from WISE ${ }^{23}$ observations obtained

\footnotetext{
$\overline{22}$ The Galaxy Evolution Explorer (GALEX) is a NASA Small Explorer-class space telescope that from 2003 to 2012 conducted an imaging survey in a farUV band (FUV, 130-190 nm) and a near-UV band (NUV, 180-280 nm). Martin et al. (2005) discuss the extragalactic science program; Morissey et al. $(2005,2007)$ discuss the on-orbit performance, survey calibration, and data products. The A. Waszczak et al. (2015, in preparation) NUV data shown here are derived from data available at http://galex.stsci.edu.

${ }^{23}$ The Wide-field Infrared Survey Explorer (WISE) is a NASA Medium Explorer-class space telescope that in 2010 conducted a cryogenic IR imaging survey in four bands: $W 1, W 2, W 3$, and $W 4$, centered at 3.4, 4.6, 12, and $22 \mu \mathrm{m}$, respectively. Wright et al. (2010) detail mission/performance; Masiero et al. (2011 and references therein) present preliminary asteroid data.
} 
Table 4

Parameters Describing PTF Light Curves with a Reliable Period or Phase Function. Byte-by-byte Description of file: ptf_asteroid_lc_parameters.txt

\begin{tabular}{|c|c|c|c|c|}
\hline Bytes & Format & Units & Label & Explanations \\
\hline$\overline{1-4}$ & I4 & $\ldots$ & $\ldots$ & Light-curve ID number ${ }^{a}$ \\
\hline $6-11$ & I6 & $\ldots$ & $\ldots$ & Asteroid number (IAU designation) \\
\hline $13-14$ & $\mathrm{I} 2$ & $\mathrm{yr}$ & $\ldots$ & Last two digits of opposition year \\
\hline $18-20$ & $\mathrm{I} 3$ & $\ldots$ & $\ldots$ & Number of observations in the light curve \\
\hline $22-26$ & F5.2 & mag & $\ldots$ & Median apparent magnitude \\
\hline $28-37$ & F10.5 & day & $t_{\min }$ & Time (MJD) of first observation \\
\hline $56-60$ & F5.2 & $\operatorname{deg}$ & $\alpha_{\max }$ & Maximum-observed phase angle \\
\hline $62-63$ & $\mathrm{I} 2$ & $\ldots$ & $\ldots$ & Number of sampled phase-angle bins of 3-deg width \\
\hline $65-68$ & F4.2 & $\cdots$ & $p$ & Reliability score from machine classifier: $0=$ bad, $1=$ good \\
\hline 70 & I1 & $\ldots$ & $\cdots$ & Manually assigned reliability flag: $0=$ bad, $1=$ good \\
\hline 72 & I1 & $\ldots$ & $\ldots$ & Period reliability flag: $0=$ bad, $1=$ good (product of two previous columns) \\
\hline $107-113$ & F7.4 & $\mathrm{mag} / \mathrm{deg}$ & $\beta$ & Phase-function parameter $\beta$ \\
\hline $115-119$ & F6.3 & mag & $C$ & Phase-function parameter $C$ \\
\hline $121-124$ & $\mathrm{~F} 4.2$ & mag & $\cdots$ & Amplitude from $G_{12}$ fit $(\max -\min )$ \\
\hline $126-134$ & F9.4 & $\mathrm{hr}$ & $P$ & Period from $G_{12}$ fit \\
\hline $136-144$ & F9.4 & $\mathrm{hr}$ & $\ldots$ & Period uncertainty from $G_{12} \mathrm{fi}$ \\
\hline $146-152$ & F7.4 & mag & $A_{11}$ & Fourier coefficient $A_{1,1}$ from G12 fit \\
\hline $154-160$ & F7.4 & mag & $A_{12}$ & Fourier coefficient $A_{1,2}$ from G12 fit \\
\hline $162-168$ & F7.4 & mag & $A_{21}$ & Fourier coefficient $A_{2,1}$ from G12 fit \\
\hline $170-176$ & F7.4 & mag & $A_{22}$ & Fourier coefficient $A_{2,2}$ from G12 fit \\
\hline $178-181$ & $\mathrm{~F} 4.2$ & $\ldots$ & $\ldots$ & Ratio of the two peak heights in folded rotation curve $\mathrm{c}^{\mathrm{c}}$ \\
\hline $183-186$ & $\mathrm{~F} 4.2$ & $\cdots$ & $\chi_{\text {red }}^{2}$ & Reduced chi-squared of the fit \\
\hline $238-241$ & $\mathrm{~F} 4.2$ & $\ldots$ & $\ldots$ & Photometry-based taxonomic index: $0=\mathrm{C}$-type, $1=\mathrm{S}$-type \\
\hline
\end{tabular}

Notes.

a ID number labels individual observations in Table 4.

${ }^{\mathrm{b}}$ Set to -1 if larger than the interval tested in grid search.

${ }^{c}$ Set to 0 if there is only one maximum in the folded light curve.

${ }^{\mathrm{d}}$ References for the IR diameters are given in the text (Appendix).

e Bond albedo only computed for objects with reliable $G_{12}$ and available diameter.

(This table is available in its entirety in machine-readable and Virtual Observatory (VO) forms.)

during its fully cryogenic mission. We only include asteroids that were detected in both of the thermal bands (W3 and $W 4)$ and that therefore have a reliable diameter estimate. Use of this diameter in Equation (15) then permits estimation of the albedo, where the W1-band albedos use the corresponding WISE photometry ( $H$ in Equation (15) being replaced with the appropriate $W 1$-band absolute magnitude).

The rightmost plot in Figure 27 shows our color index's relationship to a near-infrared color from the ground-based 2MASS (Skrutskie et al. 2006). Serendipitous asteroid detections were extracted from 2MASS by Sykes $(2000,2010)$ and include fluxes in $J$ band $(1.25 \mu \mathrm{m}), H$ band $(1.65 \mu \mathrm{m} —$ not to be confused with the absolute visible magnitude $H$, used elsewhere in this work), and $K$ band $(2.17 \mu \mathrm{m})$.

Figure 28 plots our color index against proper orbital elements retrieved from the Asteroids Dynamic Site (AstDyS; Knežević \& Milani 2012), revealing the distinct colors of dynamical families and the overall transition from $\mathrm{S}$ to $\mathrm{C}$ types with increasing semimajor axis. These are similar to the plots of Parker et al. (2008), which is not surprising given that the majority of the asteroids' color indices are based on SDSS data alone. Of the 32,5023 asteroids with a defined color index, there are 30,508 with proper orbital elements which are represented in Figure 28. 

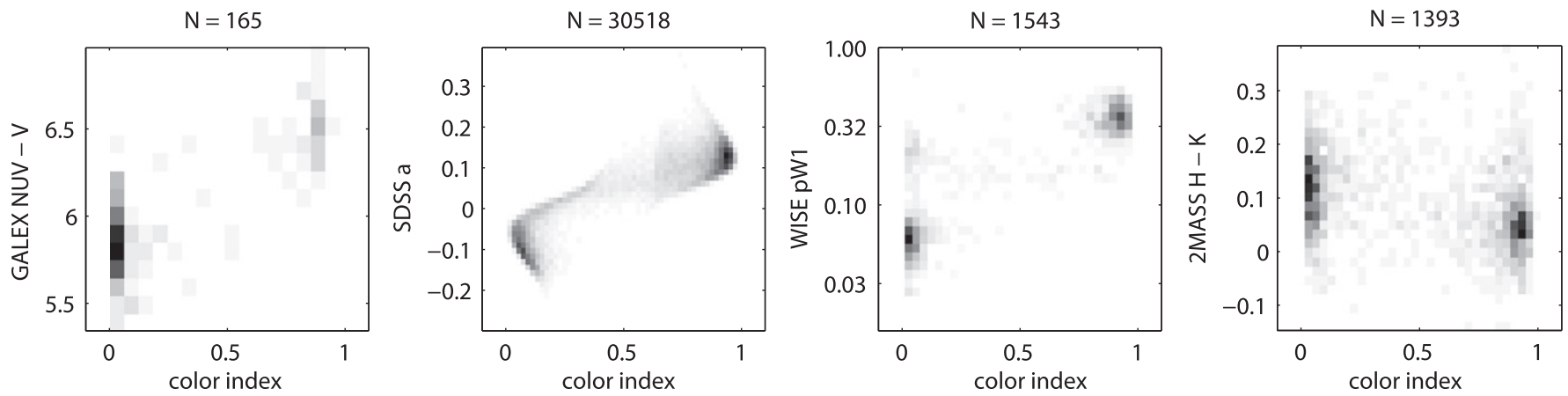

Figure 27. Relationship between various asteroid surface measurements (from the UV to near-IR) and this work's visible-color-derived C/S color index. See text for descriptions of data the data sets used here, and accompanying references.

Table 5

Parameters Describing PTF Light Curves with a Reliable Period or Phase Function. Byte-by-byte Description of File: ptf_asteroid_lc_observations.txt

\begin{tabular}{|c|c|c|c|c|}
\hline Bytes & Format & Units & Label & Explanations \\
\hline $1-4$ & I4 & $\ldots$ & $\ldots$ & Light curve ID number ${ }^{a}$ \\
\hline $6-15$ & F10.5 & day & $\tau$ & $\begin{array}{l}\text { Light-time-corrected observation } \\
\text { epoch }\end{array}$ \\
\hline $17-26$ & F10.7 & $\mathrm{AU}$ & $r$ & Heliocentric distance \\
\hline $28-37$ & F10.7 & $\mathrm{AU}$ & $\Delta$ & Geocentric distance \\
\hline $39-43$ & F5.2 & deg & $\alpha$ & Solar phase angle \\
\hline $45-50$ & F6.3 & mag & $R$ or $g$ & Apparent magnitude $^{\mathrm{b}}$ \\
\hline $52-56$ & F5.3 & mag & $\ldots$ & Uncertainty in apparent magnitude \\
\hline $58-62$ & F5.3 & mag & $\cdots$ & $\begin{array}{l}\text { Uncertainty in apparent magnitude } \\
\text { with cosmic-error }\end{array}$ \\
\hline 64-69 & F6.3 & mag & $\cdots$ & $\begin{array}{l}\text { Magnitude corrected for distance and } \\
G_{12} \text { phase function }\end{array}$ \\
\hline $71-76$ & F6.3 & mag & $\cdots$ & $\begin{array}{l}\text { Magnitude corrected for distance and } \\
\text { rotation }\left(G_{12} \text { fit }\right)\end{array}$ \\
\hline $78-83$ & F6.3 & mag & $\cdots$ & Residual with respect to the $G_{12}$ fit \\
\hline $85-89$ & $\mathrm{~F} 4.1$ & $\cdots$ & $\cdots$ & Rotational phase from 0 to 1 ( $G_{12}$ fit) \\
\hline
\end{tabular}

${ }^{\text {a }}$ ID number also corresponds to the line number in Table 4.

${ }^{\mathrm{b}}$ Filter/band is specified in Table 4.

(This table is available in its entirety in machine-readable and Virtual Observatory (VO) forms.)

\section{A.2 Compilation of IR-derived Diameters}

Similar to how we combined several surveys' colorimetric data in the previous section, here we compile thermal-infraredderived diameter estimates from four surveys. Our aim is again to provide the largest possible sample for comparison with PTF-derived light curve data. Just as SDSS is the main contributor of colorimetry overall but suffers from incompleteness for large/bright asteroids, analogously WISE provides the vast majority of IR-based diameter measurements but levels off at $\sim 80 \%$ completeness at the bright end (Figure 29). We thus supplement WISE with diameter data from the Infrared Astronomical Satellite (IRAS; Matson et al. 1986; Tedesco et al. 2002), the Mid-Course Space Experiment (MSX; Tedesco et al. 2002), and AKARI Usui et al. (2011, 2014) compares several of these different data sets in terms of coverage and accuracy. As we did when defining the color index, asteroids occurring in multiple IR surveys are assigned the varianceweighted average diameter.

Regarding the WISE data in particular, we again use only those diameters that resulted from a thermal fit constrained by
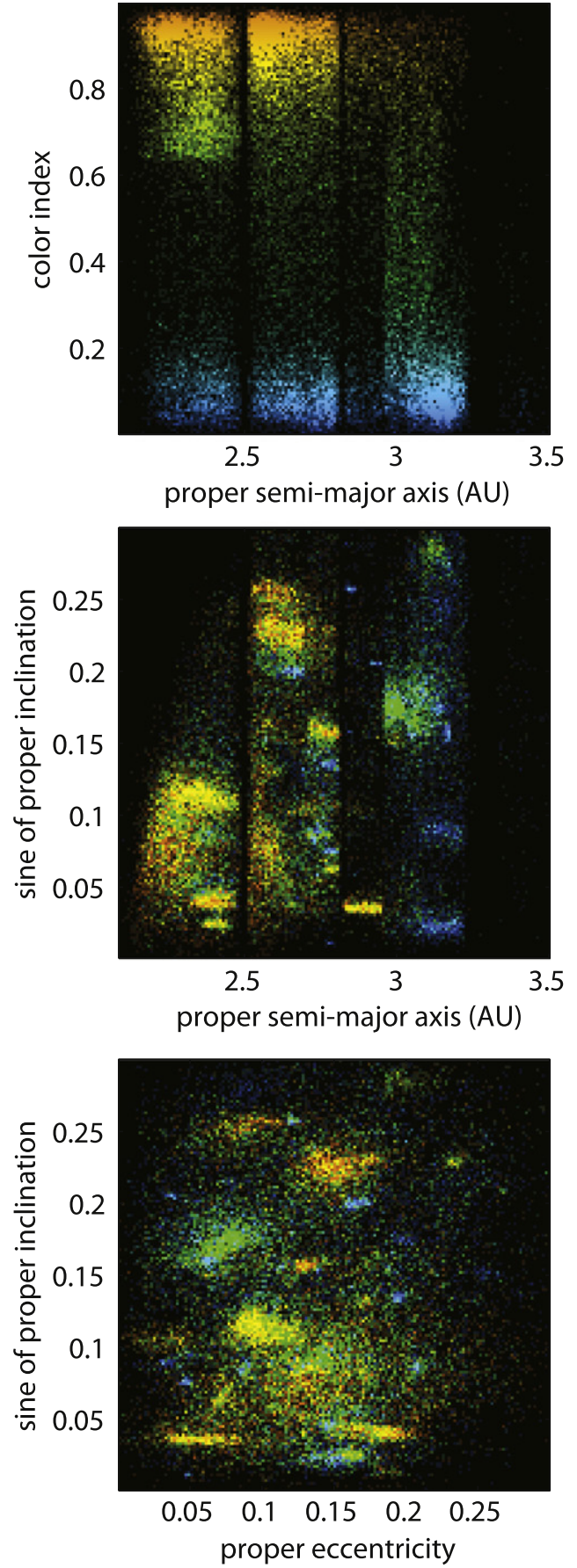

Figure 28. Relationship between proper orbital elements and this work's visible-color-derived $\mathrm{C} / \mathrm{S}$ color index for 30,508 asteroids. 

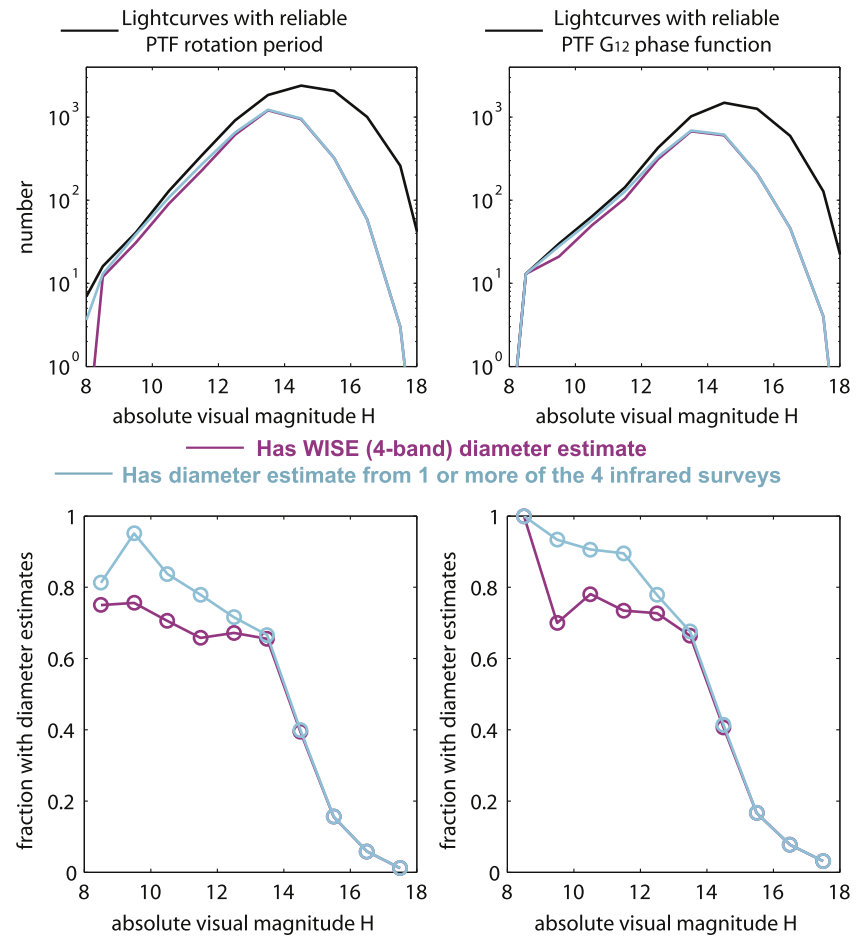

Figure 29. Fraction of PTF light curves with thermal-IR-based diameter estimates available, for both the reliable-period and reliable- $G_{12}$ sets of light curves.

fluxes in all four WISE bands during the cryogenic mission. Furthermore, we use the latest (revised) diameter estimates published by Masiero et al. (2014), which adopted an improved thermal modeling technique first discussed by Grav et al. (2012).

\section{A.3 Light-curve Data Tables}

The online version of this article includes two electronic tables containing the derived light-curve parameters and the individual photometric observations in each light curve. Tables 4 and 5 describe the columns and formatting of these tables, which include data on all reliable-period light curves, as well as those having amplitudes less than 0.1 mag and sampling in five or more 3-deg-wide phase-angle bins (which have reliable $G_{12}$ fits). Using these tables, one can produce plots of the PTF light curves we have analyzed in this work.

\section{REFERENCES}

Abazajian, K. N., Adelman-McCarthy, J. K., Agüeros, M. A., et al. 2009, ApJS, 182, 543

Belskaya, I. N., \& Shevchenko, V. G. 2000, Icar, 147, 94

Bertin, E., \& Arnouts, S. 1996, A\&AS, 117, 393

Bezdec, J. C. 1981, Pattern Recognition with Fuzzy Objective Function Algorithms (New York: Plenum)

Blomme, J., Sarro, L. M., O’Donovan, F. T., et al. 2011, MNRAS, 418, 96

Bloom, J., Richards, J. W., Nugent, P. E., et al. 2012, PASP, 124, 1175

Bodewitts, D., Kelley, M. S., Li, J. Y., et al. 2011, ApJL, 733, L3

Bottke, W. F., Vokrouhlicky, D., Rubincam, D. P., \& Nesvorný, D. 2006, AREPS, 34, 157

Bowell, E., Chapman, C. R., Gradie, J. C., Morrison, D., \& Zellner, B. 1978, Icar, 35, 313

Bowell, E., Hapke, B., Domingue, D., et al. 1989, in Asteroids II, ed. R. P. Binzel, T. Gehrels, \& M. Shapley Matthews (Tucson, AZ: Univ. Arizona Press), 524

Breiman, L. 2001, Machine Learning, 45, 5
Breiman, L., \& Cutler, A. 2004, Random Forests ${ }^{\mathrm{TM}}$, http://oz.berkeley.edu/ breiman/RandomForests/

Breiman, L., Friedman, J. H., Olshen, R. A., \& Stone, C. I. 1984, Classification and Regression Trees (Belmont, CA: Wadsworth)

Brett, D. R., West, R. G., \& Wheatley, P. J. 2004, MNRAS, 353, 369

Burns, J. A., \& Safronov, V. S. 1973, MNRAS, 165, 403

Bus, S. J., \& Binzel, R. P. 2002, Icar, 158, 106

Bus, S. J., \& Binzel, R. P. 2003, PDSS, 1

Bus, S. J., Vilas, F., \& Barucci, M. 2002, in Asteroids III, ed. W. F. Bottke, Jr et al. (Tucson, AZ: Univ. Arizona Press), 169

Carbognani, A. 2010, Icar, 205, 497

Carry, B. 2012, P\&SS, 73, 98

Cellino, A., Hestroffer, D., Tanga, P., Mottola, S., \& Dell'Oro, A. 2009, A\&A, 506,935

Chandrasekhar, S. 1969, Ellipsoidal Figures of Equilibrium (New Haven, CT: Yale Univ. Press)

Chang, C., Ip, W., Lin, H., et al. 2014a, ApJ, 788, 17

Chang, C., Waszczak, A., Lin, H., et al. 2014b, ApJL, 791, L35

Chapman, C. R., \& Gaffey, M. 1979, Asteroids (Tucson, AZ: Univ. Arizona Press)

Chapman, C. R., Gaffey, M., \& McFadden, L. 1993, PDSS, 27

Chiu, S. 1994, Journal of Intelligent and Fuzzy Systems, 2, 267

Cikota, S., Ortiz, J. L., Cikota, A., Morales, N., \& Tancredi, G. 2014, A\&A, 562, A94

Deb, S., \& Singh, H. P. 2009, A\&A, 507, 1729

Gehrels, T. 1956, ApJ, 123, 331

Giorgini, J. D., Yeomans, D. K., Chamberlin, A. B., et al. 1996, BAAS, 28,1158

Grav, T., Mainzer, A. K., Bauer, J. M., Masiero, J. R., \& Nugent, C. R. 2012, ApJ, 759, 49

Hanuš, J., \& Ďurech, J. 2012, P\&SS, 73, 75

Hanuš, J., Ďurech, J., Brož, M., et al. 2013, A\&A, 551, A67

Hapke, B. 2012, Theory of Reflectance and Emittance Spectroscopy (2nd ed.; Cambridge, UK: Cambridge Univ. Press

Harris, A. W., \& Burns, J. A. 1979, Icar, 40, 115

Harris, A. W., Pravec, P., Galád, A., et al. 2014, Icar, 235, 55

Harris, A. W., \& Young, J. W. 1988, BAAS, 20, 865

Harris, A. W., \& Young, J. W. 1989, Icar, 81, 314

Harris, A. W., Young, J. W., Bowell, E., et al. 1989a, Icar, 77, 171

Harris, A. W., Young, J. W., Contreiras, L., et al. 1989b, Icar, 81, 365

Harris, A. W., Young, J. W., Dockweiler, T., \& Gibson, J. 1992, Icar, 95, 115

Helfenstein, P., \& Veverka, J. 1989, in Asteroids II, ed. R. P. Binzel, T. Gehrels, \& M. Shapley Matthews (Tucson, AZ: Univ. Arizona Press), 557

Hogg, D. W., Bovy, J., \& Lang, D. 2010, arXiv:1008.4686

Ivezić, Ž., Lupton, R. H., Jurić, M., et al. 2002, AJ, 124, 2943

Ivezić, Z., The SDSS Collaboration, et al. 2010, PDSS, 9

Kaasalainen, M. 2004, A\&A, 422, L39

Kaasalainen, M., Torppa, J., \& Muinonen, K. 2001, Icar, 153, 37

Knežević, Z., \& Milani, A. 2012, in IAU Joint Discussion 7: Space-Time Reference Systems for Future Research, Asteroids Dynamic SiteAstDySP18

Kron, R. G. 1980, ApJS, 43, 305

Kulkarni, S. R. 2013, ATel, 4807, 1

Laher, R. R., Surace, J., Grillmair, C. J., et al. 2014, PASP, 126, 674

Law, N., Dekany, R. G., Rahmer, G., et al. 2010, Proc. SPIE, 7735, 77353M

Law, N., Kulkarni, S. R., Dekany, R. G., et al. 2009, PASP, 121, 1395

Lazarro, D., Angeli, C. A., Carvano, J. M., et al. 2004, Icar, 172, 179

Levitan, D., Fulton, B. J., Groot, P. J., et al. 2011, ApJ, 739, 68

Lowry, S. C., Fitzsimmons, A., Pravec, P., et al. 2007, Sci, 316, 272

Lupishko, D. F., Tupieva, F. A., Velichko, F. P., Kiselev, N. N., \& Chernova, G. P. 1981, AVest, 15, 25

Mahabal, A., Djorgovski, S. G., Turmon, M., et al. 2008, AN, 329, 288

Marsden, B. G. 1986, in Minor Planet Circ. Nos. 10193 and 10194, Notes from the IAU General Assembly

Martin, D. C., Fanson, J., Schiminovich, D., et al. 2005, ApJ, 619, 1

Masci, F. J., Hoffman, D. I., Grillmair, C. J., \& Cutri, R. M. 2014, AJ, 148, 21

Masiero, J., Grav, T., Mainzer, A. K., et al. 2014, ApJ, 791, 121

Masiero, J., Jedicke, R., Ďurech, J., et al. 2009, Icar, 204, 145

Masiero, J., Mainzer, A. K., Grav, T., et al. 2011, ApJ, 741, 68

Massey, F. J. 1951, J. Am. Stat. Assoc., 46, 68

Matson, D. L., Veeder, G. J., Tedesco, E. F., Lebofsky, L. A., \& Walker, R. G. 1986, AdSpR, 6, 47

Miller, A., Bloom, J. S., Richards, J., et al. 2014, ApJ, 798, 122

Morissey, P., Conrow, T., Barlow, T. A, et al. 2007, ApJS, 173, 682

Morissey, P., Schiminovich, D., Barlow, T. A., et al. 2005, ApJ, 619, 1 
Muinonen, K., Belskaya, I. N., Cellino, A., et al. 2010, Icar, 209, 542

Ofek, E. O. 2014, ascl soft, record ascl:1407.005

Ofek, E. O., Frail, D. A., Breslauer, B., et al. 2011, ApJ, 740, 65

Ofek, E. O., Laher, R., Law, N., et al. 2012a, PASP, 124, 62

Ofek, E. O., Laher, R., Surace, J., et al. 2012b, PASP, 124, 854

Oszkiewicz, D. A., Bowell, E., Wasserman, L. H., et al. 2012, Icar, 219,283

Oszkiewicz, D. A., Muinonen, K., Bowell, E., et al. 2011, JQSRT, 112,1919

Park, M., Oh, H.-S., \& Kim, D. 2013, PASP, 125, 470

Parker, A., Ivezić, Ž., Jurić, M., et al. 2008, Icar, 198, 138

Polishook, D., \& Borsch, N. 2009, Icar, 199, 319

Polishook, D., Ofek, E. O., Waszczak, A., et al. 2012, MNRAS, 421, 2094

Pravec, P., Harris, A. W., \& Michalowski, T. 2002, in Asteroids III, ed. W. F. Bottke, Jr. et al. (Tucson, AZ: Univ. Arizona Press), 113

Pravec, P., Harris, A. W., Vokrouhlicky̌, D., et al. 2008, Icar, 197, 497

Rau, A., Kulkarni, S. R., Law, N. M., et al. 2009, PASP, 103, 1334

Salo, H. 1987, Icar, 70, 37

Sanchez, J. A., Reddy, V., Nathues, A., et al. 2012, Icar, 220, 36

Seager, S. 2010, Exoplanet Atmospheres: Physical Processes (Princeton, NJ: Princeton Univ. Press)

Shevchenko, V. G. 1997, SolSyR, 31, 219

Shevchenko, V. G., Belskaya, I. N., Slyusarev, I. G., et al. 2012, Icar, 217, 202

Shevchenko, V. G., Slyusarev, I. G., Belskaya, I. N., \& Chiorny, V. G. 2013, LPICo, 44, 2706

Skrutskie, M. F., Cutri, R. M., Stiening, R., et al. 2006, AJ, 131, 1163
Steinberg, E., \& Sari, R. 2015, AJ, 149, 124

Stetson, P. B. 1996, PASP, 108, 851

Sykes, M. V., Cutri, R. M., Fowler, J. W., et al. 2000, Icar, 146, 161

Sykes, M. V., Cutri, R. M., Skrutskie, M. F., Kirkpatrick, D. J., et al. 2010 PDSS, 125

Tedesco, E. F. 1995, PDSS, 30

Tedesco, E. F., Egan, M. P., \& Price, S. D. 2002, AJ, 124, 583

Tedesco, E. F., Noah, P. V., Noah, M., \& Price, S. D. 2002, AJ, 123, 1056

Usui, F., Hasegawa, S., Ishiguro, M., Müller, T. G., Ootsubo, T., et al. 2014 PASJ, 66, 56

Usui, F., Kuroda, D., Müller, T. G., et al. 2011, PASJ, 63, 1117

Walsh, K. J., Morbidelli, A., Raymond, S. N., O’Brien, D. P., \& Mandell, A. M. 2011, Natur, 475, 206

Warner, B., Harris, A. W., \& Pravec, P. 2009, Icar, 202, 134

Waszczak, A., Ofek, E. O., Aharonson, O., et al. 2013, MNRAS, 433, 3115

Williams, G. V. 2012, PhD thesis, Open University, Milton Keynes, U.K.

Woźniak, P. R., Williams, S. J., Vestrand, W. T., \& Gupta, V. 2004, AJ, 128,2965

Wright, E. L., Eisenhardt, P., Mainzer, A. K., et al. 2010, AJ, 140, 1868

Xu, S., Binzel, R. P., Burbine, T. H., \& Bus, S. J. 1995, Icar, 115, 1

Xu, S., Binzel, R. P., Burbine, T. H., \& Bus, S. J. 1996, PDSS, 168

York, D. G., Adelman, J., Anderson, S. F., et al. 2000, AJ, 120, 1579

Zacharias, N. 2010, AJ, 139, 2208

Zellner, B., Tholen, D. J., \& Tedesco, E. F. 1998, Icar, 61, 355

Zellner, B., Tholen, D. J., \& Tedesco, E. F. 2009, PDSS, 100 